\title{
Enantiospecific Synthesis of Nepetalactones by One-Step Oxidative NHC Catalysis
}

\section{Wacharee Harnying*, Jörg-M. Neudörfl, ${ }^{\dagger}$ and Albrecht Berkessel*}

Department of Chemistry (Organic Chemistry), University of Cologne, Greinstr. 4, 50939 Cologne, Germany; Fax: (+49) 221-4705102

*E-mail: berkessel@uni-koeln.de, wharnyin@uni-koeln.de

${ }^{\dagger} \mathrm{X}$-ray crystallography

\section{Supporting Information}

\section{Table of contents}

1. General 2

2. Synthesis of the nitrophenylhydrazines $\mathbf{1 0} \quad 2$

2.1 General procedure 1 (GP-1) for the nitration of anilines to give 92

2.2 General procedure 2 (GP-2) for the synthesis of hydrazines $\mathbf{1 0}$ from $\mathbf{9} 4$

3. Synthesis of the triazolium salts 13-19 6

3.1 Synthesis of the benzamides $11 \quad 6$

3.2 General procedure 3 (GP-3) for the three-step synthesis of triazolium salts $\quad 7$

4. Synthesis of 8-oxocitronellal (5) from citronellol 10

5. One-step synthesis of nepetalactones (1a) from $\mathbf{5} \quad 11$

5.1 Procedure for the oxidative NHC-catalyzed transformation of $\mathbf{5}$ to $\mathbf{1 a} \quad 11$

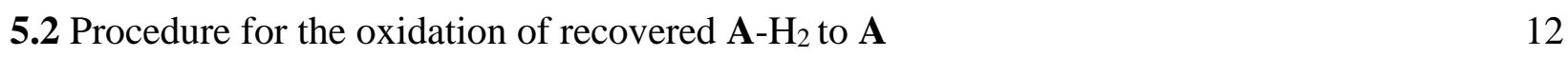

6. HPL chromatograms for the determination of the enantiomeric composition of citronellol 13

7. Gas chromatograms for the determination of the enantiomeric composition of 1a 14

8. NMR spectra 16

9. X-ray data 36 


\section{General}

Nuclear magnetic resonance $\left({ }^{1} \mathrm{H},{ }^{13} \mathrm{C}\right)$ spectra were recorded on a Bruker Avance 300 instrument $\left({ }^{1} \mathrm{H}\right.$ : $\left.300.13 \mathrm{MHz},{ }^{13} \mathrm{C}: 75.46 \mathrm{MHz}\right)$ at ambient temperature. Chemical shifts $(\delta)$ are reported in parts per million (ppm) relative to tetramethylsilane (TMS) or solvent residual signals. The following abbreviations were used for chemical shift multiplicities in ${ }^{1} \mathrm{H}$ NMR spectra: $\mathrm{br}=$ broad, $\mathrm{s}=$ singlet, $\mathrm{d}$ $=$ doublet, $\mathrm{t}=$ triplet, $\mathrm{q}=$ quartet, sept $=$ septet, $\mathrm{m}=$ multiplet. IR spectra were recorded on a Shimadzu IR Affinity-1 FT-IR spectrometer. GC-MS analyses were done on a Hewlett Packard HP 6890 Series Plus gas chromatograph, injector and autosampler, HP 5973 Series mass-selective detector operated in electron-impact (EI) mode with an ionization energy of $75 \mathrm{eV}$ and all other parameters set at autotune values, using $\mathrm{H}_{2}$ as carrier gas and a HP-5 MS column (30 m x $0.25 \mathrm{~mm} \mathrm{ID}, 0.25 \mu \mathrm{m}$ film thickness): temperature program (Std 50): $50{ }^{\circ} \mathrm{C}, 5 \mathrm{~min} ; 20{ }^{\circ} \mathrm{C} / \mathrm{min}$ to $280{ }^{\circ} \mathrm{C}, 280{ }^{\circ} \mathrm{C}, 10 \mathrm{~min}$, unless otherwise stated. ESI-mass spectra were measured on an Agilent 1100 Series LC/MSD instrument with a G1312A binary pump, G1313A autosampler and G1956A mass-selective detector. GC analyses were done on an Agilent 6890N Network gas chromatograph, injector and autosampler, and flame ionization detector, using $\mathrm{N}_{2}$ as carrier gas. Elemental analyses were performed using an Elementar Vario EL elemental analyzer. Optical rotations were recorded on an Anton Paar MCP 200 polarimeter. Melting points were determined on a Büchi apparatus and are uncorrected.

$(S)-(-)-\beta$-Citronellol (Merck), $(R)-(+)-\beta$-citronellol (Sigma Aldrich), 2,4,6-trimethylaniline (Alfa Aesar), 2,6-diisopropylaniline (Alfa Aesar), 2,6-dimethylaniline (Sigma Aldrich), 2,6-diethylaniline (Sigma Aldrich), fuming nitric acid (90\%, Acros), 3-nitrophenylhydrazine hydrochloride (TCI), triethyl orthoformate (Acros), chlorobenzene (Acros), and $\mathrm{HCl}$ in dioxane (ca. $4 \mathrm{M}$, TCI) were purchased from the suppliers indicated and used as received. Diisopropyl ethyl amine (DIPEA) was distilled over $\mathrm{CaH}_{2}$ under Ar. Anhydrous THF and $\mathrm{MeCN}$ were dried over Na/benzophenone and $\mathrm{CaH}_{2}$, respectively, and distilled under Ar.

\section{Synthesis of the nitrophenylhydrazines $\mathbf{1 0}$}
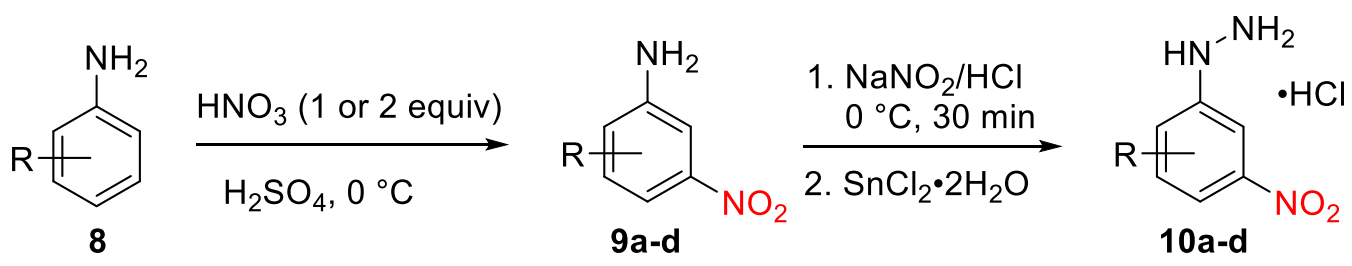

\subsection{General procedure 1 (GP-1) for the nitration of anilines to give 9: ${ }^{1}$}

In a three-necked round-bottom flask, a solution of aniline 8 (150 mmol, 1.0 equiv) in conc. $\mathrm{H}_{2} \mathrm{SO}_{4}$ $(100 \mathrm{~mL})$ was cooled in an ice-salt bath. Fuming nitric acid $(90 \%, d=1.5,1.0$ or 2.0 equiv) was slowly added dropwise, the internal temperature was controlled not to exceed $10^{\circ} \mathrm{C}$. After the addition was complete, stirring of the reaction mixture was continued in the ice bath for $1 \mathrm{~h}$. The mixture was then poured into ice (ca. $500 \mathrm{~mL}$ ) and cooled down in an ice-water bath. The mixture was made slightly basic by slow addition of $\mathrm{NaOH}(6 \mathrm{~N})$, keeping the temperature below $25^{\circ} \mathrm{C}$. The resulting

\footnotetext{
1 (a) Noelting, E.; Stoecklin, L. Ber. Dtsch. Chem. Ges. 1891, 24, 564-572. (b) Bergman, J.; Sand, P. Tetrahedron 1990, 46, 6085-6112.
} 
precipitate (except for 9d, see below) was collected by suction filtration, washed with water and dried to give 9, which was used in the next step without further purification.

2,4,6-Trimethyl-3,5-dinitroaniline (9a). According to GP-1, this product was obtained from the<smiles>Cc1c(N)c([N+](=O)[O-])c([N+](=O)[O-])c([N+](=O)[O-])c1[N+](=O)[O-]</smiles>
reaction between 2,4,6-trimethylaniline (20.3 g, $150 \mathrm{mmol}, 1.0$ equiv) and $90 \%$ nitric acid (15.0 mL, $300 \mathrm{mmol}, 2.0$ equiv) in conc. $\mathrm{H}_{2} \mathrm{SO}_{4}(100 \mathrm{~mL})$ at $0{ }^{\circ} \mathrm{C}$ for $1 \mathrm{~h}$ and then at $\mathrm{rt}$ for $1 \mathrm{~h}$. After filtration, the product $9 \mathrm{a}$ was obtained as a yellowbrown powder $(19.4 \mathrm{~g}, 86 \mathrm{mmol}, 57 \%)$, which was used in the next step without further purification; ${ }^{1} \mathrm{H}$ NMR $\left(300 \mathrm{MHz}, \mathrm{DMSO}-d_{6}\right): \delta=5.74\left(\mathrm{~s}, 2 \mathrm{H}, \mathrm{NH}_{2}\right), 2.02$ (s, 6H, 2xCH $), 1.94$ (s, 3H, $\left.\mathrm{CH}_{3}\right)$; ${ }^{13} \mathrm{C}$ NMR (75 MHz, DMSO- $\left.d_{6}\right): \delta=150.1$ (ArC), $145.2(2 \times A r C)$, $113.2(2 \times A r C), 105.0(\mathrm{ArC}), 12.6\left(2 \mathrm{xCH}_{3}\right), 11.3\left(\mathrm{CH}_{3}\right)$; IR (ATR): $\tilde{v}\left[\mathrm{~cm}^{-1}\right]=3499(\mathrm{w}), 3410(\mathrm{~m})$, 2988 (w), 2884 (w), 1632 (m), 1510 (s), 1475 (m), 1362 (s), 1076 (m), 862 (m), 804 (m), 669 (m); GC-MS: $t_{\mathrm{R}}(\operatorname{Std} 50)=14.1 \mathrm{~min}, \mathrm{~m} / z=225\left(\mathrm{M}^{+}, 100 \%\right), 208,191,178,162,148,132,120,106,91$, $77,65,54$.

2,4,6-Trimethyl-3-nitroaniline (9b). According to GP-1, this product was obtained from the reaction<smiles>Cc1cc([N+](=O)[O-])c([N+](=O)[O-])c([N+](=O)[O-])c1N</smiles>
between 2,4,6-trimethylaniline (20.3 g, $150 \mathrm{mmol}, 1.0$ equiv) and 90\% nitric acid (7.0 mL, $150 \mathrm{mmol}, 1.0$ equiv) in conc. $\mathrm{H}_{2} \mathrm{SO}_{4}(100 \mathrm{~mL})$ at $0{ }^{\circ} \mathrm{C}$ for $1 \mathrm{~h}$. After filtration, the product 9b was obtained as a yellow powder $(26.3 \mathrm{~g}, 146 \mathrm{mmol}$, $97 \%$ ), which was used in the next step without further purification; ${ }^{1} \mathrm{H}$ NMR (300 $\left.\mathrm{MHz}_{\mathrm{CDCl}}\right): \delta=6.82(\mathrm{~s}, 1 \mathrm{H}, \mathrm{ArH}), 3.67\left(\mathrm{~s}, 2 \mathrm{H}, \mathrm{NH}_{2}\right), 2.16\left(\mathrm{~s}, 6 \mathrm{H}, 2 \mathrm{xCH}_{3}\right), 2.05$ (s, 3H, $\left.\mathrm{CH}_{3}\right) ;{ }^{13} \mathrm{C} \mathrm{NMR}\left(75 \mathrm{MHz}, \mathrm{CDCl}_{3}\right): \delta=141.5(\mathrm{ArC}), 130.0(\mathrm{ArCH}), 123.8$ (ArC), 117.7 (ArC), $112.7(\mathrm{ArC}), 17.6\left(\mathrm{CH}_{3}\right), 16.6\left(\mathrm{CH}_{3}\right), 12.3\left(\mathrm{CH}_{3}\right)$; IR (ATR): $\tilde{v}\left[\mathrm{~cm}^{-1}\right]=3414$ (m), 3350 (w), 2928 (w), 2862 (w), 1632 (m), 1508 (s), 1474 (m), 1358 (s), 1306 (m), 1049 (m), 851 $(\mathrm{m}), 800(\mathrm{~m})$; GC-MS: $t_{\mathrm{R}}(\operatorname{Std} 50)=12.5 \mathrm{~min}, \mathrm{~m} / z=180\left(\mathrm{M}^{+}, 100 \%\right), 163,150,135,118,108,91,77$, $65,54$.

2,6-Dimethyl-3-nitroaniline (9c). According to GP-1, this product was obtained from the reaction<smiles>Cc1ccc([N+](=O)[O-])c([N+](=O)[O-])c1N</smiles>
between 2,6-dimethylaniline (30.0 g, $247 \mathrm{mmol}, 1.0$ equiv) and 90\% nitric acid (12.5 mL, $270 \mathrm{mmol}, 1.1$ equiv) in conc. $\mathrm{H}_{2} \mathrm{SO}_{4}(180 \mathrm{~mL})$ at $10-15^{\circ} \mathrm{C}$ for $1 \mathrm{~h}$. After filtration, the product 9c was obtained as a yellow powder $(36.4 \mathrm{~g}, 219 \mathrm{mmol}$, $89 \%$ ), which was used in the next step without further purification; ${ }^{1} \mathrm{H}$ NMR (300 $\left.\mathrm{MHz} \mathrm{CDCl}_{3}\right): \delta=7.16(\mathrm{~d}, J=8.2 \mathrm{~Hz}, 1 \mathrm{H}, \mathrm{ArH}), 7.01(\mathrm{~d}, J=8.2 \mathrm{~Hz}, 1 \mathrm{H}, \operatorname{ArH})$, 3.79 (br s, $\left.2 \mathrm{H}, \mathrm{NH}_{2}\right), 2.28\left(\mathrm{~s}, 3 \mathrm{H}, \mathrm{CH}_{3}\right), 2.23\left(\mathrm{~s}, 3 \mathrm{H}, \mathrm{CH}_{3}\right) ;{ }^{13} \mathrm{C} \mathrm{NMR}\left(75 \mathrm{MHz}, \mathrm{CDCl}_{3}\right): \delta=144.2$ (ArC), $127.7 \mathrm{ArCH}), 126.3(\mathrm{ArC}), 115.2(\mathrm{ArC}), 113.4(\mathrm{ArCH}), 18.1\left(\mathrm{CH}_{3}\right), 12.9\left(\mathrm{CH}_{3}\right)$; IR (ATR): $\tilde{v}$ $\left[\mathrm{cm}^{-1}\right]=3485(\mathrm{w}), 3421(\mathrm{~m}), 3389(\mathrm{w}), 3350(\mathrm{~m}), 2980(\mathrm{w}), 2951(\mathrm{w}), 2914(\mathrm{w}), 2851(\mathrm{w}), 1638(\mathrm{~m})$, 1593 (m), 1508 (s), 1458 (m), 1350 (s), 1321 (s), 1277 (m), 1219 (m), 1042 (m), 868 (m), 802 (s), 741 (s); GC-MS: $t_{\mathrm{R}}(\operatorname{Std} 50)=12.4 \mathrm{~min}, \mathrm{~m} / z=166\left(\mathrm{M}^{+}, 100 \%\right), 149,136,121,104,94,77,65,51$.

2,6-Diethyl-3-nitroaniline (9d). According to GP-1, this product was obtained from the reaction<smiles>CCc1ccc([N+](=O)[O-])c(N)c1CC</smiles>

9d between 2,6-diethylaniline (22.4 g, $150 \mathrm{mmol}, 1.0$ equiv) and 90\% nitric acid (7.0 $\mathrm{mL}, 150 \mathrm{mmol}, 1.0$ equiv) in conc. $\mathrm{H}_{2} \mathrm{SO}_{4}(100 \mathrm{~mL})$ at $0{ }^{\circ} \mathrm{C}$ for $1 \mathrm{~h}$. After basicification with $\mathrm{NaOH}$, the mixture was extracted with EtOAc. The combined organic layers were washed with water, brine, dried over $\mathrm{Mg}_{2} \mathrm{SO}_{4}$, and filtered. After 
removal of the solvent, the product 9d was obtained as a yellow oil (29.1 g, $150 \mathrm{mmol}, 100 \%)$, which was used in the next step without further purification; ${ }^{1} \mathrm{H}$ NMR $\left(300 \mathrm{MHz}, \mathrm{CDCl}_{3}\right): \delta=7.16(\mathrm{~d}, J=$ $8.3 \mathrm{~Hz}, 1 \mathrm{H}, \mathrm{ArH}), 7.02(\mathrm{~d}, J=8.3 \mathrm{~Hz}, 1 \mathrm{H}, \mathrm{ArH}), 3.95\left(\mathrm{~s}, 2 \mathrm{H}, \mathrm{NH}_{2}\right), 2.66\left(\mathrm{q}, J=7.5 \mathrm{~Hz}, 2 \mathrm{H}, \mathrm{CH}_{2}\right)$, $2.55\left(\mathrm{q}, J=7.5 \mathrm{~Hz}, 2 \mathrm{H}, \mathrm{CH}_{2}\right), 1.30\left(\mathrm{t}, J=7.4 \mathrm{~Hz}, 3 \mathrm{H}, \mathrm{CH}_{3}\right), 1.28\left(\mathrm{t}, J=7.5 \mathrm{~Hz}, 3 \mathrm{H}, \mathrm{CH}_{3}\right) ;{ }^{13} \mathrm{C} \mathrm{NMR}$ $\left(75 \mathrm{MHz}, \mathrm{CDCl}_{3}\right): \delta=149.4(\mathrm{ArC}), 143.2(\mathrm{ArC}), 132.2(\mathrm{ArC}), 125.8(\mathrm{ArCH}), 121.0(\mathrm{ArC}), 113.6$ $(\mathrm{ArCH}), 24.6\left(\mathrm{CH}_{2}\right), 20.7\left(\mathrm{CH}_{2}\right), 12.5\left(\mathrm{CH}_{3}\right), 12.4\left(\mathrm{CH}_{3}\right)$; IR (ATR): $\tilde{v}\left[\mathrm{~cm}^{-1}\right]=3497(\mathrm{w}), 3410(\mathrm{~m})$, 2968 (m), 2938 (w), 2876 (m), 1732 (w), 1626 (m), 1512 (s), 1468 (m), 1431 (m), 1344 (s), 1955 (m), $814(\mathrm{~s}), 735(\mathrm{~m}) \mathrm{cm}^{-1}$; GC-MS: $t_{\mathrm{R}}(\operatorname{Std} 50)=13.1 \mathrm{~min}, \mathrm{~m} / \mathrm{z}=194\left(\mathrm{M}^{+}, 100 \%\right), 177,162,149,135$, $117,108,91,77,65,51$.

\subsection{General procedure 2 (GP-2) for the synthesis of hydrazines 10 from 9 :}

In a three-necked round-bottom flask equipped with an overhead stirrer, a suspension of aniline 9 (1.0 equiv) in conc. $\mathrm{HCl}$ (ca. $2 \mathrm{~mL} / \mathrm{mmol}$ ) was cooled in an ice-salt bath. A solution of $\mathrm{NaNO}_{2}(1.1$ equiv) in water (ca. $0.18 \mathrm{~mL} / \mathrm{mmol}$ ) was added dropwise, keeping the internal reaction temperature below $5{ }^{\circ} \mathrm{C}$. After the addition was complete, stirring of the reaction mixture was continued in the ice bath for $30 \mathrm{~min}$. A solution of $\mathrm{SnCl}_{2} \bullet 2 \mathrm{H}_{2} \mathrm{O}$ (2.5 equiv) in conc. $\mathrm{HCl}$ (ca. $0.25 \mathrm{~mL} / \mathrm{mmol}$ ) was added dropwise such that the temperature stayed below $5{ }^{\circ} \mathrm{C}$. After completion of the addition, stirring of the reaction mixture was continued at $0{ }^{\circ} \mathrm{C}$ for $1 \mathrm{~h}$ and then at $\mathrm{rt}$ for $24 \mathrm{~h}$. The precipitate was collected by suction filtration, and washed with $4 \mathrm{~N} \mathrm{HCl}$ (Important note: the product is soluble in water!) and then with $\mathrm{Et}_{2} \mathrm{O}$, providing the hydrazine hydrochloride $\mathbf{1 0} \bullet \mathrm{HCl}$, which can be used in the next step without further purification.

To obtain the free hydrazine 10, a suspension of the hydrochloride salt $10 \bullet \mathrm{HCl}$ in water (ca. 1 $\mathrm{mL} / \mathrm{mmol}$ ) was treated with cold $10 \% \mathrm{NaOH}$ (aq.) at $0{ }^{\circ} \mathrm{C}$ until slightly basic. After stirring at $0{ }^{\circ} \mathrm{C}$ for $1 \mathrm{~h}$, the solid was collected by suction filtration and washed with water and $\mathrm{Et}_{2} \mathrm{O}$ to give the free hydrazine 10, which was used in the next step without further purification. The product should be stored at temperature below $4{ }^{\circ} \mathrm{C}$.

2,4,6-Trimethyl-3,5-dinitrophenylhydrazine (10a). According to GP-2, the reaction of 9a (13.5 g, $60.0 \mathrm{mmol}, 1.0$ equiv) in conc. $\mathrm{HCl}(100 \mathrm{~mL})$ with a solution of $\mathrm{NaNO}_{2}(4.5 \mathrm{~g}, 66.0 \mathrm{mmol}, 1.1$ equiv) in water $(15 \mathrm{~mL})$, followed by the addition of a solution of $\mathrm{SnCl}_{2} \bullet 2 \mathrm{H}_{2} \mathrm{O}(33.8 \mathrm{~g}, 149.8 \mathrm{mmol}, 2.5$ equiv) in conc. $\mathrm{HCl}(50 \mathrm{~mL})$ yielded $\mathbf{1 0 a} \cdot \mathrm{HCl}(12.1 \mathrm{~g}, 43.7 \mathrm{mmol}, 73 \%)$ as a yellow solid: ${ }^{1} \mathrm{H}$ NMR

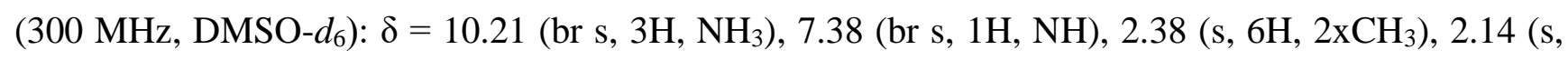
$\left.3 \mathrm{H}, \mathrm{CH}_{3}\right) ;{ }^{13} \mathrm{C} \mathrm{NMR}\left(75 \mathrm{MHz}, \mathrm{DMSO}-d_{6}\right): \delta=149.9$ (2xArC), 141.1 (ArC), 130.2 (2xArC), 119.4 (ArC), $13.5\left(2 \times \mathrm{CH}_{3}\right), 12.2\left(\mathrm{CH}_{3}\right)$.

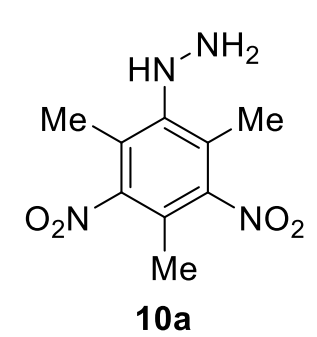

10a•HCl $(9.8 \mathrm{~g}, 35.4 \mathrm{mmol})$ was treated with $\mathrm{NaOH}$ to give 10a $(8.2 \mathrm{~g}, 34.1$ mmol, 96\%) as a beige solid; ${ }^{1} \mathrm{H}$ NMR $\left(300 \mathrm{MHz}, \mathrm{CDCl}_{3}\right): \delta=6.30(\mathrm{~s}, 1 \mathrm{H}, \mathrm{NH})$, $4.35\left(\mathrm{~s}, 2 \mathrm{H}, \mathrm{NH}_{2}\right), 2.20\left(\mathrm{~s}, 6 \mathrm{H}, 2 \mathrm{x} \mathrm{CH}_{3}\right), 2.02(\mathrm{~s}, 3 \mathrm{H}) . ;{ }^{13} \mathrm{C} \mathrm{NMR}(75 \mathrm{MHz}$, $\left.\mathrm{CDCl}_{3}\right): \delta=150.5$ (ArC), $149.2(2 \mathrm{xArC}), 121.6(2 \mathrm{xArC}), 111.5(\mathrm{ArC}), 13.8$ $\left(2 \mathrm{xCH}_{3}\right), 11.9\left(\mathrm{CH}_{3}\right)$; IR (ATR): $\tilde{v}\left[\mathrm{~cm}^{-1}\right]=3352(\mathrm{w}), 3327(\mathrm{w}), 2980(\mathrm{w}), 2901$ (w), 1520 (s), 1389 (m), 1360 (m), 1294 (w), 1142 (w), 1070 (w), 1016 (w), 866 (m), $779(\mathrm{~m}), 679(\mathrm{~m})$; GC-MS: $t_{\mathrm{R}}(\mathrm{Std} 50)=14.6 \mathrm{~min}, \mathrm{~m} / z=240\left(\mathrm{M}^{+}, 100 \%\right), 224,206,189,178$, $160,149,131,117,105,91,77,65,51$. 
2,4,6-Trimethyl-3-nitrophenylhydrazine (10b). According to GP-2, the reaction of 9b (13.5 g, 74.9 mmol, 1.0 equiv) in conc. $\mathrm{HCl}(120 \mathrm{~mL})$ with a solution of $\mathrm{NaNO}_{2}(5.7 \mathrm{~g}, 82.6 \mathrm{mmol}, 1.1$ equiv $)$ in water $(15 \mathrm{~mL})$, followed by the addition of a solution of $\mathrm{SnCl}_{2} \bullet 2 \mathrm{H}_{2} \mathrm{O}(42.4 \mathrm{~g}, 187.5 \mathrm{mmol}, 2.5 \mathrm{equiv})$ in conc. $\mathrm{HCl}(50 \mathrm{~mL})$ yielded $\mathbf{1 0 b \bullet} \mathrm{HCl}(17.0 \mathrm{~g}, 73.4 \mathrm{mmol}, 98 \%)$ as a yellow solid: ${ }^{1} \mathrm{H}$ NMR (300 MHz, DMSO-d6): $\delta=9.97$ (br s, 3H, NH 3 ), 7.21 (s, 1H, ArH), 7.00 (br s, 1H, NH), 2.45 (s, 3H, $\mathrm{CH}_{3}$ ), $2.30\left(\mathrm{~s}, 3 \mathrm{H}, \mathrm{CH}_{3}\right), 2.20\left(\mathrm{~s}, 3 \mathrm{H}, \mathrm{CH}_{3}\right) ;{ }^{13} \mathrm{C} \mathrm{NMR}\left(75 \mathrm{MHz}, \mathrm{DMSO}-d_{6}\right): \delta=150.3(\mathrm{ArC}), 139.2(\mathrm{ArC})$, 138.4 (ArC), 130.7 (ArCH), $127.3(\mathrm{ArC}), 126.9(\mathrm{ArC}), 18.0\left(\mathrm{CH}_{3}\right), 16.4\left(\mathrm{CH}_{3}\right), 12.9\left(\mathrm{CH}_{3}\right)$.

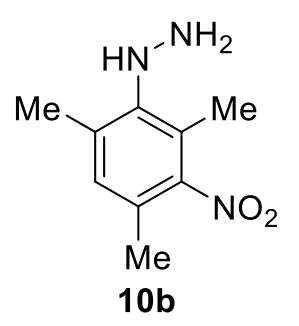

$\mathbf{1 0 b} \cdot \mathrm{HCl}(16.0 \mathrm{~g}, 69.1 \mathrm{mmol})$ was treated with $\mathrm{NaOH}$ to give $\mathbf{1 0 b}(10.5 \mathrm{~g}, 53.8$ mmol, 78\%) as a pale yellow solid; ${ }^{1} \mathrm{H}$ NMR $\left(300 \mathrm{MHz}, \mathrm{CDCl}_{3}\right): \delta=7.03(\mathrm{~s}, 1 \mathrm{H}$, ArH), 6.17 (br s, 3H, $\mathrm{NHNH}_{2}$ ), 2.33 (s, 3H, $\left.\mathrm{CH}_{3}\right), 2.19$ (s, 3H, $\left.\mathrm{CH}_{3}\right), 2.13$ (s, 3H, $\left.\mathrm{CH}_{3}\right) ;{ }^{13} \mathrm{C}$ NMR $\left(75 \mathrm{MHz}, \mathrm{CDCl}_{3}\right): \delta=150.5$ (ArC), 142.9 (ArC), 135.0 (ArC), 130.4 (ArCH), 124.1 (ArC), $123.8(\mathrm{ArC}), 18.1\left(\mathrm{CH}_{3}\right), 16.2\left(\mathrm{CH}_{3}\right), 13.1\left(\mathrm{CH}_{3}\right) ; \mathrm{IR}$ (ATR): $\tilde{v}\left[\mathrm{~cm}^{-1}\right]=3358(\mathrm{w}), 3291(\mathrm{~m}), 3181(\mathrm{w}), 2972(\mathrm{w}), 2934(\mathrm{w}), 1607(\mathrm{w})$, 1512 (s, br), 1460 (m), 1375 (m), 1360 (s), 1277 (m), 1123 (s), 887 (s), 837 (m), 779 (s), 743 (s).; GCMS: $t_{\mathrm{R}}(\operatorname{Std} 50)=13.1 \mathrm{~min}, \mathrm{~m} / \mathrm{z}=195\left(\mathrm{M}^{+}, 100 \%\right), 179,161,149,132,117,106,91,77,65,53$.

2,6-Dimethyl-3-nitrophenylhydrazine (10c). According to GP-2, the reaction of 9c $(17.0 \mathrm{~g}, 102.3$ mmol, 1.0 equiv) in conc. $\mathrm{HCl}(150 \mathrm{~mL})$ with a solution of $\mathrm{NaNO}_{2}(7.7 \mathrm{~g}, 111.6 \mathrm{mmol}, 1.1$ equiv $)$ in water $(15 \mathrm{~mL})$, followed by the addition of a solution of $\mathrm{SnCl}_{2} \bullet 2 \mathrm{H}_{2} \mathrm{O}(59.1 \mathrm{~g}, 261.9 \mathrm{mmol}, 2.5$ equiv) in conc. $\mathrm{HCl}(75 \mathrm{~mL})$ yielded $10 \mathrm{c} \bullet \mathrm{HCl}(14.7 \mathrm{~g}, 67.5 \mathrm{mmol}, 66 \%)$ as a beige solid; ${ }^{1} \mathrm{H}$ NMR (300 MHz, DMSO- $\left.d_{6}\right): \delta=10.04\left(\mathrm{~s}, 3 \mathrm{H}, \mathrm{NH}_{3}\right), 7.74(\mathrm{~d}, J=8.4 \mathrm{~Hz}, 1 \mathrm{H}, \mathrm{ArH}), 7.34(\mathrm{~d}, J=8.4 \mathrm{~Hz}, 1 \mathrm{H}$, $\mathrm{ArH}), 7.11(\mathrm{~s}, 1 \mathrm{H}, \mathrm{NH}), 2.52\left(\mathrm{~s}, 3 \mathrm{H}, \mathrm{CH}_{3}\right), 2.49\left(\mathrm{~s}, 3 \mathrm{H}, \mathrm{CH}_{3}\right) ;{ }^{13} \mathrm{C} \mathrm{NMR}\left(75 \mathrm{MHz}, \mathrm{DMSO}-d_{6}\right): \delta=$ 149.4 (ArC), 142.4 (ArC), 141.5 (ArC), 130.3 (ArC), $129.3 \mathrm{ArCH}), 122.9(\mathrm{ArCH}), 19.1\left(\mathrm{CH}_{3}\right), 14.5$ $\left(\mathrm{CH}_{3}\right)$.

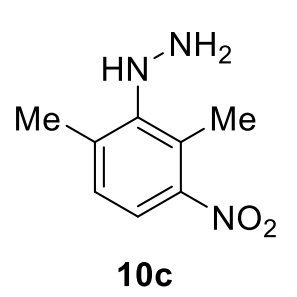

10c• $\mathrm{HCl}(10.0 \mathrm{~g}, 46.0 \mathrm{mmol})$ was treated with $\mathrm{NaOH}$ to give $10 \mathrm{c}(6.5 \mathrm{~g}, 35.9 \mathrm{mmol}$, $78 \%$ ) as a beige solid; ${ }^{1} \mathrm{H}$ NMR (300 MHz, DMSO- $\left.d_{6}\right): \delta=7.37(\mathrm{~d}, J=7.6 \mathrm{~Hz}, 1 \mathrm{H}$, ArH), 7.14 (d, $J=7.5 \mathrm{~Hz}, 1 \mathrm{H}, \mathrm{ArH}), 6.10$ (br s, 1H, NH), 6.10 (br s, 2H, $\mathrm{NH}_{2}$ ), 2.37 $\left(\mathrm{s}, 6 \mathrm{H}, 2 \mathrm{xCH}_{3}\right) ;{ }^{13} \mathrm{C} \mathrm{NMR}\left(75 \mathrm{MHz}, \mathrm{DMSO}-d_{6}\right): \delta=149.6(\mathrm{ArC}), 148.7(\mathrm{ArC})$, $134.7(\mathrm{ArC}), 128.1(\mathrm{ArCH}), 124.0(\mathrm{ArC}), 117.2(\mathrm{ArCH}), 18.6\left(\mathrm{CH}_{3}\right), 14.6\left(\mathrm{CH}_{3}\right)$; IR (ATR): $\tilde{v}\left[\mathrm{~cm}^{-1}\right]=3350(\mathrm{w}), 3300(\mathrm{~m}), 3181(\mathrm{w}), 2976(\mathrm{w}), 1591(\mathrm{w}), 1516(\mathrm{~s}), 1354(\mathrm{~s}), 1283(\mathrm{~m})$, $1258(\mathrm{~m}), 1126(\mathrm{~m}), 1047(\mathrm{~m}), 912(\mathrm{~m}), 835(\mathrm{~m}), 812(\mathrm{~m}), 793(\mathrm{~m}), 743(\mathrm{~m})$; GC-MS: $t_{\mathrm{R}}(\operatorname{Std}$ 50) = $13.0 \mathrm{~min}, \mathrm{~m} / z=181\left(\mathrm{M}^{+}, 100 \%\right), 165,147,134,119,106,91,77,65,51$.

2,6-Diethyl-3-nitrophenylhydrazine hydrochloride (10d•HCl). According to GP-2, the reaction of

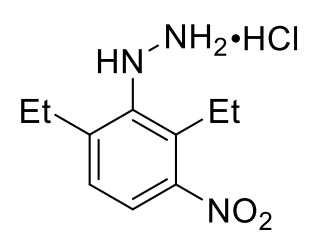

$10 \mathrm{~d} \cdot \mathrm{HCl}$

9d (13.6 g, $70.0 \mathrm{mmol}, 1.0$ equiv) in conc. $\mathrm{HCl}(100 \mathrm{~mL})$ with a solution of $\mathrm{NaNO}_{2}(5.3 \mathrm{~g}, 76.8 \mathrm{mmol}, 1.1$ equiv) in water $(15 \mathrm{~mL})$, followed by the addition of a solution of $\mathrm{SnCl}_{2} \bullet 2 \mathrm{H}_{2} \mathrm{O}(39.6 \mathrm{~g}, 175.5 \mathrm{mmol}, 2.5$ equiv) in conc. $\mathrm{HCl}$ (50 $\mathrm{mL})$ yielded $10 \mathrm{~d} \bullet \mathrm{HCl}(14.9 \mathrm{~g}, 60.6 \mathrm{mmol}, 87 \%)$ as a cream coloured solid: ${ }^{1} \mathrm{H}$ NMR (300 MHz, DMSO-d $): \delta=10.08\left(\mathrm{~s}, 3 \mathrm{H}, \mathrm{NH}_{3}\right), 7.75(\mathrm{~d}, J=8.4 \mathrm{~Hz}, 1 \mathrm{H}$, $\operatorname{ArH}), 7.38(\mathrm{~d}, J=8.5 \mathrm{~Hz}, 1 \mathrm{H}, \mathrm{ArH}), 7.00(\mathrm{~s}, 1 \mathrm{H}, \mathrm{NH}), 2.88\left(\mathrm{~m}, 4 \mathrm{H}, 2 \mathrm{xCH}_{2}\right), 1.20$ (t, $J=7.5 \mathrm{~Hz}, 3 \mathrm{H}$, $\left.\mathrm{CH}_{3}\right), 1.13\left(\mathrm{t}, J=7.4 \mathrm{~Hz}, 3 \mathrm{H}, \mathrm{CH}_{3}\right) ;{ }^{13} \mathrm{C} \mathrm{NMR}\left(75 \mathrm{MHz}, \mathrm{DMSO}-d_{6}\right): \delta=148.6$ (ArC), $146.9(\operatorname{ArC})$, 140.9 (ArC), $135.0(\mathrm{ArC}), 127.2(\mathrm{ArCH}), 122.9(\mathrm{ArCH}), 23.9\left(\mathrm{CH}_{2}\right), 20.6\left(\mathrm{CH}_{2}\right), 15.1\left(\mathrm{CH}_{3}\right), 14.1$ 
$\left(\mathrm{CH}_{3}\right)$; IR (ATR): $\tilde{v}\left[\mathrm{~cm}^{-1}\right]=3277(\mathrm{w}), 2974(\mathrm{~m}), 2876(\mathrm{~m}), 2662(\mathrm{w}), 1601(\mathrm{~m}), 1522(\mathrm{~s}), 1462(\mathrm{~m})$, $1356(\mathrm{~m}), 1119(\mathrm{w}), 883(\mathrm{w}), 839(\mathrm{~m}), 797$ (m), $741(\mathrm{~m})$; ESI-MS (positive): $\mathrm{m} / \mathrm{z}=210\left(\mathrm{M}^{+}-\mathrm{Cl}\right.$, $100 \%)$.

\section{Synthesis of the triazolium salts 13-19}

\subsection{Synthesis of the benzamides 11}

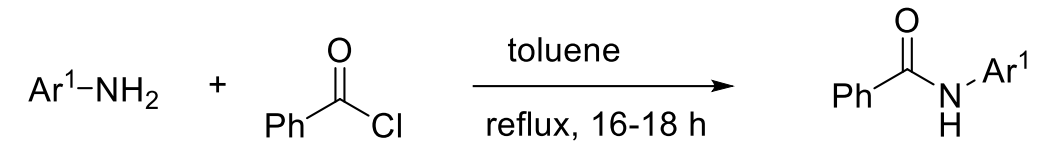

$$
\begin{aligned}
& A r^{1}=\text { Mes, Dipp 11a, } A r^{1}=\text { Mes } \\
& \text { 11b, } A r^{1}=D i p p
\end{aligned}
$$

N-(2,4,6-Trimethylphenyl)benzamide (11a): 2,4,6-Trimethylaniline (32.0 mL, $228 \mathrm{mmol}, 1.0$ equiv) was dissolved in toluene (200 mL, HPLC grade). Benzoyl chloride (30.0 mL, $260 \mathrm{mmol}, 1.1$ equiv) was added dropwise at room temperature. The resulting suspension was refluxed for $16 \mathrm{~h}$ under a drying tube containing $\mathrm{CaCl}_{2}$. The reaction mixture was cooled down in an ice-bath and water (20 $\mathrm{mL}$ ) was added. The resulting suspension was stirred for $30 \mathrm{~min}$. The solid was collected by suction filtration and washed with EtOAc, affording the benzamide 11a as a colorless solid (52.44 g, 219 mmol, $96 \%$ ); mp 207-208 ${ }^{\circ} \mathrm{C}$ (lit. $\left.{ }^{2} 205-206{ }^{\circ} \mathrm{C}\right) ;{ }^{1} \mathrm{H}$ NMR $\left(300 \mathrm{MHz}, \mathrm{DMSO}-d_{6}\right): \delta=9.68(\mathrm{~s}, 1 \mathrm{H}$, $\mathrm{NH}), 7.99-8.01(\mathrm{~m}, 2 \mathrm{H}, \mathrm{PhH}), 7.52-7.57$ (m, 3H, PhH), 6.93 (s, 2H, ArH), 2.26 (s, 3H, $\left.\mathrm{CH}_{3}\right), 2.15$ (s, $\left.6 \mathrm{H}, 2 \mathrm{xCH}_{3}\right) ;{ }^{13} \mathrm{C}$ NMR $\left(75 \mathrm{MHz}, \mathrm{DMSO}-d_{6}\right): \delta=165.0(\mathrm{C}=\mathrm{O}), 135.6(\mathrm{ArC}), 135.3(2 \mathrm{xArC}), 134.5$ (ArC), 132.7 (ArC), $131.4(\mathrm{ArCH}), 128.4(2 \mathrm{xArCH}), 128.3(2 \mathrm{xArCH}), 127.5(2 \mathrm{xArCH}), 20.5\left(\mathrm{CH}_{3}\right)$, $18.0\left(2 \mathrm{xCH}_{3}\right)$; IR (ATR): $\tilde{v}\left[\mathrm{~cm}^{-1}\right]=3269(\mathrm{~m}), 2949(\mathrm{w}), 2916(\mathrm{w}), 1638(\mathrm{~s}), 1601(\mathrm{~m}), 1578(\mathrm{~m})$, 1514 (s), 1485 (s), 1308 (m), 1292 (s), 1223 (m), 1074 (m), 1028 (m), 851 (s), 802 (m), 710 (s), 687 $(\mathrm{s}), 665(\mathrm{~s}) ; \mathrm{GC}-\mathrm{MS}: t_{\mathrm{R}}(\operatorname{Std} 50)=15.1 \mathrm{~min}, \mathrm{~m} / z=239\left(\mathrm{M}^{+}, 100 \%\right), 134,105,91,77,51$.

N-(2,6-Diisopropylphenyl)benzamide (11b): 2,6-Diisopropylaniline (37.6 g, $212 \mathrm{mmol}, 1.0$ equiv) was dissolved in toluene (200 ml, HPLC grade). Benzoyl chloride (26.0 mL, $226 \mathrm{mmol}, 1.1$ equiv) was added dropwise at room temperature. The resulting suspension was refluxed for $18 \mathrm{~h}$ under a drying tube containing $\mathrm{CaCl}_{2}$. The reaction mixture was cooled down in an ice-bath and water (20 $\mathrm{mL}$ ) was added. The resulting suspension was stirred for $30 \mathrm{~min}$. The solid was collected by suction filtration and washed with hexane, affording the benzamide 11b as a colorless solid (53.2 $\mathrm{g}, 189$ mmol, $89 \%)$; mp 264-265 ${ }^{\circ} \mathrm{C}$ (lit. ${ }^{3}$ 265.4.-265.6 $\left.{ }^{\circ} \mathrm{C}\right) ;{ }^{1} \mathrm{H}$ NMR $\left(300 \mathrm{MHz}, \mathrm{CDCl}_{3}\right): \delta=7.92(\mathrm{~d}, J=$ $7.6 \mathrm{~Hz}, 2 \mathrm{H}, \mathrm{ArH}), 7.61-7.46$ (m, 3H, ArH), $7.38-7.31$ (m, 2H, ArH+NH), 7.23 (d, J= 7.6 Hz, 2H, ArH), 3.14 (hept, $J=6.9 \mathrm{~Hz}, 2 \mathrm{H}, 2 \mathrm{xCH}), 1.22\left(\mathrm{~d}, J=6.9 \mathrm{~Hz}, 12 \mathrm{H}, 4 \mathrm{xCH}_{3}\right) ;{ }^{13} \mathrm{C} \mathrm{NMR}(75 \mathrm{MHz}$, DMSO- $\left.d_{6}\right): \delta=166.1(\mathrm{C}=\mathrm{O}), 146.2(2 \times \mathrm{ArC}), 134.4(\mathrm{ArC}), 132.8(\mathrm{ArC}), 131.4(\mathrm{ArCH}), 128.4$ (2xArCH), $127.6(2 \mathrm{xArCH}), 127.5(\mathrm{ArCH}), 122.9(2 \mathrm{xArCH}), 28.2(2 \mathrm{xCH}), 23.5\left(\mathrm{CH}_{3}\right), 23.3\left(\mathrm{CH}_{3}\right)$; IR (ATR): $\tilde{v}\left[\mathrm{~cm}^{-1}\right]=3262(\mathrm{w}), 2963(\mathrm{~m}), 1639$ (s), 1518 (s), 1487 (s), $1458(\mathrm{~m}), 1285$ (s), $1256(\mathrm{~m})$, $797(\mathrm{~m}), 745(\mathrm{~m}), 692(\mathrm{~s}), 658(\mathrm{~m})$; GC-MS: $t_{\mathrm{R}}(\operatorname{Std} 50)=14.6 \mathrm{~min}, \mathrm{~m} / \mathrm{z}=281\left(\mathrm{M}^{+}\right), 264,238$ (100\%), 223, 208, 176, 160, 146, 130, 117, 105, 91, 77, 51.

\footnotetext{
${ }^{2}$ Fan, W.; Yang, Y.; Lei, J.; Jiang, Q.; Zhou, W. J. Org. Chem. 2015, 80, 8782-8789.

${ }^{3}$ Gong, C.; Ding, H.; Lu, C.; Zhao, B.; Yao, Y. Dalton Trans. 2017, 46, 6031-6038.
} 


\subsection{General procedure $3(\mathrm{GP}-3)$ for the three-step synthesis of triazolium salts}

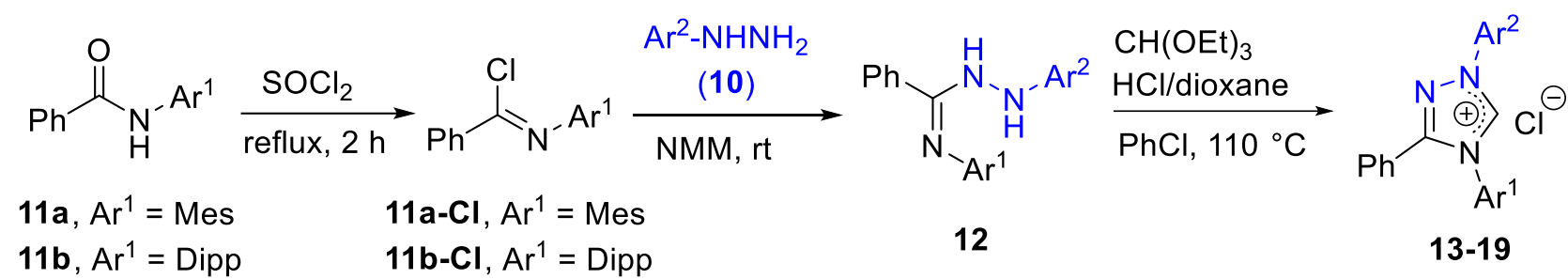

The benzamide 11 (1.0 equiv) was refluxed in $\mathrm{SOCl}_{2}$ (excess) for $2 \mathrm{~h}$. The excess of $\mathrm{SOCl}_{2}$ was distilled off in vacuo and colleccted in a trap cooled with liquid nitrogen. The residue was further dried in high vacuum to give the corresponding benzimidoyl chloride, which was used in the next step without further purification. The imidoyl chlorides $\mathbf{1 1 a}-\mathbf{C l}$ and $\mathbf{1 1 b}-\mathbf{C l}$ were also prepared on large scale and stored below $4^{\circ} \mathrm{C}$ for use in the next step.

The hydrazine $\mathbf{1 0}$ (1.0 equiv) or $\mathbf{1 0 \bullet} \mathrm{HCl}$ (1.2 equiv) and dry THF were added to the benzimidoyl chloride (1.0 eqiuv) under Ar atmosphere. The mixture was cooled in an ice bath, followed by the addition of $N$-methylmorpholine (NMM, 1.1-1.8 equiv). The reaction mixture was stirred at $0{ }^{\circ} \mathrm{C}$ for $30 \mathrm{~min}$ and then at $\mathrm{rt}$ for $24 \mathrm{~h}$. The volatiles were removed on a rotary evaporator. The residue was diluted with water and extracted with DCM. The combined organic layers were washed with water, brine, dried over $\mathrm{Na}_{2} \mathrm{SO}_{4}$, and filtered. After removal of the solvent, the crude product $\mathbf{1 2}$ was directly subjected to the final ring closing step.

To the crude 12, in a round-bottom flask, was successively added chlorobenzene, triethyl orthoformate, and anhydrous $\mathrm{HCl}$ (4 $\mathrm{M}$ in dioxane, 1-2 equiv). The reaction flask was immersed in an oil bath at $110{ }^{\circ} \mathrm{C}$, and the mixture was stirred open to air for $1-16 \mathrm{~h}$. The reaction mixture was then concentrated on a rotary evaporator at $60^{\circ} \mathrm{C}$. The residue was washed with $\mathrm{Et}_{2} \mathrm{O}$ by vigorously stirring for $1 \mathrm{~h}$ and the $\mathrm{Et}_{2} \mathrm{O}$ phase was removed. The residue was further triturated with EtOAc with vigorous stirring for at least $2 \mathrm{~h}$. The resulting solid was collected by suction filtration and washed with EtOAc (trituration with EtOAc can be repeated several times), furnishing the desired triazolium salt (13-19).

\section{1-(2,4,6-Trimethyl-3,5-dinitrophenyl)-4-(2,4,6-trimethylphenyl)-3-phenyl-1H-1,2,4-triazol-4-ium}

chloride (13). According to GP-3, the pre-formed 11a-Cl (5.1 g, $20.0 \mathrm{mmol}, 1.0$ equiv) was

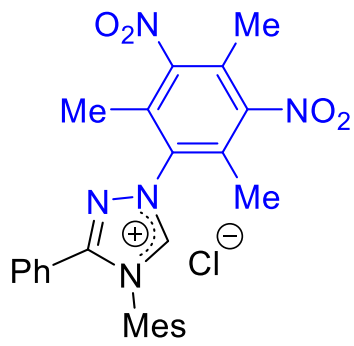

13 condensed with $\mathbf{1 0 a}$ ( $4.8 \mathrm{~g}, 20.0 \mathrm{mmol}, 1.0$ equiv) in the presence of NMM $(2.9 \mathrm{~mL}, 26.0 \mathrm{mmol}, 1.3$ equiv) in THF $(50 \mathrm{~mL})$, followed by the reaction with $\mathrm{CH}(\mathrm{OEt})_{3}(10.0 \mathrm{~mL}, 60.0 \mathrm{mmol}, 3.0$ equiv) and $\mathrm{HCl} /$ dioxane $(4 \mathrm{M}, 7.5 \mathrm{~mL}$, $30.0 \mathrm{mmol}, 1.5$ equiv) in $\mathrm{PhCl}(8.0 \mathrm{~mL})$ at $110{ }^{\circ} \mathrm{C}$ for $16 \mathrm{~h}$. After several triturations with EtOAc, 13 was obtained as a brown solid (1.0 g, $2.0 \mathrm{mmol}$, 10\%): $\mathrm{mp} 266-267{ }^{\circ} \mathrm{C} ;{ }^{1} \mathrm{H} \mathrm{NMR}\left(300 \mathrm{MHz}, \mathrm{CDCl}_{3}\right): \delta=13.57$ (s, $\left.1 \mathrm{H}, \mathrm{NCHN}\right)$, 7.63 (s, 1H, ArH), 7.48 (s, 4H, ArH), 7.12 (s, 2H, ArH), 2.40 (s, 3H, CH $)$, $2.33\left(\mathrm{~s}, 9 \mathrm{H}, 3 \mathrm{xCH}_{3}\right), 2.15\left(\mathrm{~s}, 6 \mathrm{H}, 2 \mathrm{xCH}_{3}\right) ;{ }^{13} \mathrm{C} \mathrm{NMR}\left(75 \mathrm{MHz}, \mathrm{CDCl}_{3}\right): \delta=154.7(\mathrm{ArC}), 150.6(\mathrm{ArC})$, 149.7 ( $\mathrm{NCHN}), 142.9$ (ArC), 133.9 (ArC), 133.7 (ArCH), 133.0 (ArC), 130.8 (2xArCH), 130.7 (ArC), $129.8(2 \times \mathrm{ArCH}), 127.7$ (2xArCH), $127.4(\mathrm{ArC}), 126.5$ (ArC), $121.5(\mathrm{ArC}), 21.3\left(\mathrm{CH}_{3}\right), 18.0\left(2 \mathrm{xCH}_{3}\right)$, $14.2\left(2 \mathrm{xCH}_{3}\right), 13.5\left(\mathrm{CH}_{3}\right)$; IR (ATR): $\tilde{v}\left[\mathrm{~cm}^{-1}\right]=2974(\mathrm{w}), 2901(\mathrm{~m}), 1605(\mathrm{w}), 1535(\mathrm{~s}), 1485(\mathrm{~m})$, 1449 (m), 1358 (m), 1233 (m), 1038 (m), 868 (m), 723 (m), 691 (s); ESI-MS (positive): $\mathrm{m} / \mathrm{z}=472$ $\left(\mathrm{M}^{+}-\mathrm{Cl}, 100 \%\right)$; elemental analysis calcd (\%) for $\mathrm{C}_{26} \mathrm{H}_{26} \mathrm{~N}_{5} \mathrm{O}_{4} \mathrm{Cl} \bullet 0.5 \mathrm{H}_{2} \mathrm{O}: \mathrm{C}, 60.41 ; \mathrm{H}, 5.26 ; \mathrm{N}, 13.55$; found: C, $60.58 ; \mathrm{H}, 5.27 ; \mathrm{N}, 13.80$. 
chloride (14). According to GP-3, $11 \mathrm{a}\left(4.8 \mathrm{~g}, 20.0 \mathrm{mmol}, 1.0\right.$ equiv) was reacted with $\mathrm{SOCl}_{2}(6.0$

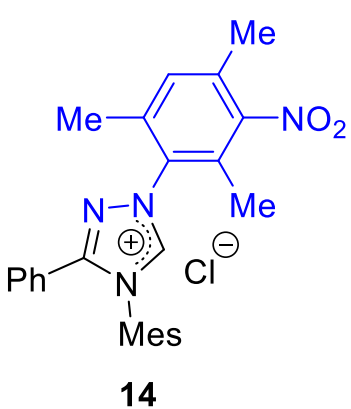
$\mathrm{mL})$, condensed with $\mathbf{1 0 b}(4.8 \mathrm{~g}, 20.0 \mathrm{mmol}, 1.0$ equiv) in the presence of NMM (2.4 mL, $22.0 \mathrm{mmol}, 1.1$ equiv) in THF $(50 \mathrm{~mL})$, followed by the reaction with $\mathrm{CH}(\mathrm{OEt})_{3}(10.0 \mathrm{~mL}, 60.0 \mathrm{mmol}, 3.0$ equiv) and $\mathrm{HCl} /$ dioxane (4 M, $7.5 \mathrm{~mL}, 30.0 \mathrm{mmol}, 1.5$ equiv) in $\mathrm{PhCl}(8.0 \mathrm{~mL})$ at $110{ }^{\circ} \mathrm{C}$ for $1 \mathrm{~h}$. After several triturations with EtOAc, 14 was obtained as a cream colored solid (3.2 g, $7.0 \mathrm{mmol}, 35 \%)$ : mp 274-275 ${ }^{\circ} \mathrm{C}$ (decomp.); ${ }^{1} \mathrm{H}$ NMR $\left(300 \mathrm{MHz}, \mathrm{CDCl}_{3}\right)$ : $\delta=13.61(\mathrm{~s}, 1 \mathrm{H}, \mathrm{NCHN}), 7.59(\mathrm{t}, J=7.1 \mathrm{~Hz}, 1 \mathrm{H}, \mathrm{ArH}), 7.54-7.37(\mathrm{~m}, 4 \mathrm{H}$, $\mathrm{ArH}), 7.28$ (s, 1H, ArH), 7.09 (s, 2H, ArH), 2.45 (s, 3H, $\left.\mathrm{CH}_{3}\right), 2.39$ (s, 6H, 2xCH 3$), 2.22\left(\mathrm{~s}, 3 \mathrm{H}, \mathrm{CH}_{3}\right)$, 2.16 (br s, 6H, 2xCH 3 ); ${ }^{13} \mathrm{C}$ NMR $\left(75 \mathrm{MHz}, \mathrm{CDCl}_{3}\right.$ ): $\delta=154.1$ (ArC), 150.4 (ArC), 149.1 (NCHN), 142.6 (ArC), 137.9 (ArC), 134.0 (ArC), 133.8 (ArC), 133.3 (ArCH), $132.0(\mathrm{ArCH}), 130.7(2 \times \mathrm{ArCH})$, 129.7 (2xArCH), $127.6(2 x \mathrm{ArCH}), 127.5(\mathrm{ArC}), 121.9(\mathrm{ArC}), 21.3\left(\mathrm{CH}_{3}\right), 18.2\left(\mathrm{CH}_{3}\right), 18.0\left(2 \mathrm{xCH}_{3}\right)$, $17.7\left(\mathrm{CH}_{3}\right), 13.5\left(\mathrm{CH}_{3}\right)$; IR (ATR): $\tilde{v}\left[\mathrm{~cm}^{-1}\right]=2996(\mathrm{w}), 2913(\mathrm{w}), 2685(\mathrm{w}), 2641(\mathrm{w}), 1601(\mathrm{w})$, 1551 (m), 1530 (s), 1478 (m), 1448 (m), 1366 (m), 1329 (w), 1252 (m), 1219 (m), 1157 (w), 1038 (m), $843(\mathrm{~m}), 781(\mathrm{~m}), 723$ (s), 691 (s); ESI-MS (positive): $\mathrm{m} / \mathrm{z}=427\left(\mathrm{M}^{+}-\mathrm{Cl}, 100 \%\right)$; elemental analysis calcd (\%) for $\mathrm{C}_{26} \mathrm{H}_{27} \mathrm{~N}_{4} \mathrm{O}_{2} \mathrm{Cl}$ : C, 67.45; H, 5.88; N, 12.10; found: C, 67.31; H, 6.12; N, 11.94 .

\section{1-(2,6-Dimethyl-3-nitrophenyl)-4-(2,4,6-trimethylphenyl)-3-phenyl-1H-1,2,4-triazol-4-ium}

chloride (15). According to GP-3, the pre-formed 11a-Cl (2.6 g, $10.0 \mathrm{mmol}, 1.0$ equiv) was

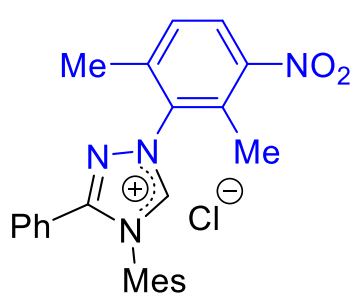

15 condensed with 10c (1.8 g, $10.0 \mathrm{mmol}, 1.0$ equiv) in the presence of NMM (1.3 $\mathrm{mL}, 12.0 \mathrm{mmol}, 1.2$ equiv) in THF $(30 \mathrm{~mL})$, followed by the reaction with $\mathrm{CH}(\mathrm{OEt})_{3}(5.0 \mathrm{~mL}, 30.0 \mathrm{mmol}, 3.0$ equiv) and $\mathrm{HCl} /$ dioxane $(4 \mathrm{M}, 3.8 \mathrm{~mL}, 15.0$ mmol, 1.5 equiv) in $\mathrm{PhCl}(5.0 \mathrm{~mL})$ at $110{ }^{\circ} \mathrm{C}$ for $1 \mathrm{~h}$. After several triturations with EtOAc, 15 was obtained as a beige solid (0.45 g, $1.0 \mathrm{mmol}, 10 \%)$ : $\mathrm{mp}$ 198-199 ${ }^{\circ} \mathrm{C} ;{ }^{1} \mathrm{H}$ NMR $\left(300 \mathrm{MHz}, \mathrm{CDCl}_{3}\right): \delta=13.58(\mathrm{~s}, 1 \mathrm{H}, \mathrm{NCHN}), 8.14(\mathrm{~d}, J$ $=8.5 \mathrm{~Hz}, 1 \mathrm{H}, \mathrm{ArH}), 7.60(\mathrm{t}, J=7.0 \mathrm{~Hz}, 2 \mathrm{H}), 7.55-7.40(\mathrm{~m}, 5 \mathrm{H}, \mathrm{ArH}), 7.11(\mathrm{~s}$, $2 \mathrm{H}, \mathrm{ArH}$ ), 2.51, 2.46, and 2.40 (each s, $3 \mathrm{x} 3 \mathrm{H}, 3 \mathrm{xCH}_{3}$ ), 2.23 and 2.12 (each br s, $2 \mathrm{x} 3 \mathrm{H}, 2 \mathrm{xCH}_{3}$ ); ${ }^{13} \mathrm{C}$ NMR (75 MHz, CDCl 3 ): $\delta=154.3(\mathrm{ArC}), 149.1$ (NCHN), 148.5 (ArC), 142.7 (ArC), 141.7 (ArC), 134.9 (ArC), 134.1 (ArC), 133.4 (ArCH), 131.4 (ArC), 130.8 (2xArCH), 129.7 (3xArCH), 127.8 (ArCH), 127.7 (2xArCH), 127.5 (ArC), 121.8 (ArC), 21.3, 18.7, 17.99, 14.94.; IR (ATR): $\tilde{v}\left[\mathrm{~cm}^{-1}\right]=$ 3292 (w), 2968 (w), 2735 (w), 1605 (w), 1551 (m), 1518 (s), 1487 (m), 1445 (m), 1350 (s), 1225 (w), $1036(\mathrm{w}), 980(\mathrm{~m}), 853(\mathrm{~m}), 833(\mathrm{~m}), 768(\mathrm{~m}), 723(\mathrm{~s})$; ESI-MS (positive): $\mathrm{m} / \mathrm{z}=413\left(\mathrm{M}^{+}-\mathrm{Cl}, 100 \%\right)$; elemental analysis calcd (\%) for $\mathrm{C}_{25} \mathrm{H}_{25} \mathrm{~N}_{4} \mathrm{O}_{2} \mathrm{Cl} \bullet 0.5 \mathrm{H}_{2} \mathrm{O}$ : C, 65.57; H, 5.72; N, 12.23; found: C, 65.18; H, 5.82; N, 11.88 . 
According to GP-3, the pre-formed 11a-Cl (5.3 g, $21.0 \mathrm{mmol}, 1.0$ equiv) was condensed with 3-

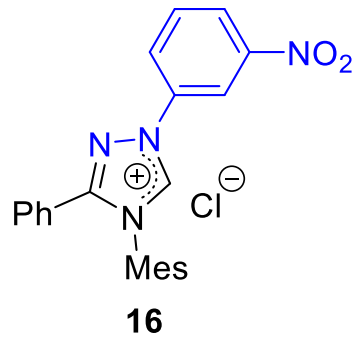
nitrophenylhydrazine hydrochloride (4.8 g, $25.0 \mathrm{mmol}, 1.2 \mathrm{equiv})$ in the presence of NMM (4.1 mL, $37.0 \mathrm{mmol}, 1.8$ equiv) in THF $(50 \mathrm{~mL})$, followed by the reaction with $\mathrm{CH}(\mathrm{OEt})_{3}(8.0 \mathrm{~mL}, 48.0 \mathrm{mmol}, 2.3$ equiv) and $\mathrm{HCl}$ in dioxane (4 M, $6.2 \mathrm{~mL}, 24.8 \mathrm{mmol}, 1.2$ equiv) in $\mathrm{PhCl}(6.0 \mathrm{~mL})$ at $110{ }^{\circ} \mathrm{C}$ for 1 h. After several triturations with EtOAc, $\mathbf{1 3}$ was obtained as a cream colored solid (4.2 g, $10.0 \mathrm{mmol}, 48 \%): \mathrm{mp}>300{ }^{\circ} \mathrm{C} ;{ }^{1} \mathrm{H}$ NMR $\left(300 \mathrm{MHz}, \mathrm{CDCl}_{3}\right): \delta=$ 13.85 (s, 1H, NCHN), 9.60 (d, $J=7.6 \mathrm{~Hz}, 1 \mathrm{H}, \mathrm{ArH}), 9.16$ (s, 1H, ArH), 8.40 $(\mathrm{d}, J=7.7 \mathrm{~Hz}, 1 \mathrm{H}), 7.88(\mathrm{t}, J=8.1 \mathrm{~Hz}, 1 \mathrm{H}, \mathrm{ArH}), 7.70-7.42(\mathrm{~m}, 5 \mathrm{H}, \mathrm{ArH}), 7.10(\mathrm{~s}, 2 \mathrm{H}, \operatorname{ArH}), 2.40(\mathrm{~s}$, $3 \mathrm{H}, \mathrm{CH}_{3}$ ), 2.17 (s, 6H, 2xCH 3 ); ${ }^{13} \mathrm{C} \mathrm{NMR}\left(75 \mathrm{MHz}, \mathrm{CDCl}_{3}\right): \delta=153.9$ (ArC), 148.8 (ArC), 145.4 ( $\mathrm{NCHN}), 142.6$ (ArC), 135.7 (ArC), 134.3 (2xArC), $133.3(\mathrm{ArCH}), 132.0(\mathrm{ArCH}), 130.7$ (2xArCH), 129.67 (2xArCH), 127.9 (2xArCH), $127.4(\mathrm{ArCH}), 125.2(\mathrm{ArCH}), 121.9(\mathrm{ArC}), 115.7$ (ArCH), 21.3 $\left(\mathrm{CH}_{3}\right), 18.2\left(2 \mathrm{xCH}_{3}\right)$; IR (ATR): $\tilde{v}\left[\mathrm{~cm}^{-1}\right]=2996(\mathrm{w}), 2909(\mathrm{w}, \mathrm{br}), 2866(\mathrm{w}), 2370(\mathrm{w}), 1605(\mathrm{w})$, 1551 (m), 1524 (s), 1491 (m), 1460 (m), 1449 (m), 1344 (s), 1254 (m), 1183 (w), 1057 (w), 986 (m), $897(\mathrm{~m}), 858(\mathrm{~m}), 752(\mathrm{~m}), 735$ (m), $720(\mathrm{~s})$; ESI-MS (positive): $\mathrm{m} / z=385\left(\mathrm{M}^{+}-\mathrm{Cl}, 100 \%\right)$; elemental analysis calcd (\%) for $\mathrm{C}_{23} \mathrm{H}_{21} \mathrm{~N}_{4} \mathrm{O}_{2} \mathrm{Cl}$ : C, 65.63; H, 5.03; N, 13.31; found: $\mathrm{C}, 65.20 ; \mathrm{H}, 5.07 ; \mathrm{N}, 13.12$.

\section{4-(2,6-Diisopropyl)phenyl)-1-(2,4,6-trimethyl-3-nitrophenyl)-3-phenyl-1H-1,2,4-triazol-4-ium}

chloride (17). According to GP-3, 11b (5.6 g, 20 mmol, 1.0 equiv) was condensed with 10b (4.8 g, 20

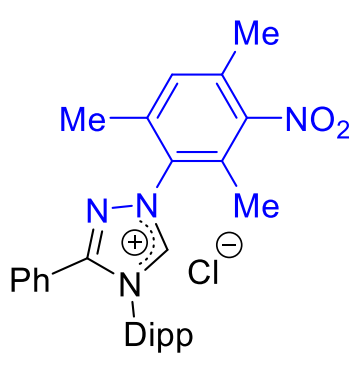

17 mmol, 1.0 equiv) in the presence of NMM (2.4 mL, $22.0 \mathrm{mmol}, 1.1$ equiv) in THF $(50 \mathrm{~mL})$, followed by the reaction with $\mathrm{CH}(\mathrm{OEt})_{3}(10.0 \mathrm{~mL}, 60 \mathrm{mmol}, 3.0$ equiv) and $\mathrm{HCl} /$ dioxane (4 M, $7.5 \mathrm{~mL}, 30 \mathrm{mmol}, 1.5$ equiv) in $\mathrm{PhCl}(8.0 \mathrm{~mL})$ at $110{ }^{\circ} \mathrm{C}$ for $1 \mathrm{~h}$. After several triturations with EtOAc, 17 was obtained as a cream colored solid (3.2 g, $6.4 \mathrm{mmol}, 32 \%)$ : mp 235-236 ${ }^{\circ} \mathrm{C} ;{ }^{1} \mathrm{H}$ NMR (300 $\mathrm{MHz}_{\mathrm{CDCl}}$ ): $\delta=13.77$ (s, 1H, NCHN), 7.66 (t, $\left.J=7.8 \mathrm{~Hz}, 1 \mathrm{H}, \mathrm{ArH}\right), 7.62-$ $7.53(\mathrm{~m}, 1 \mathrm{H}, \mathrm{ArH}), 7.50-7.36(\mathrm{~m}, 6 \mathrm{H}, \mathrm{ArH}), 7.30$ (s, 1H, ArH), 2.43 (s, 3H, $\left.\mathrm{CH}_{3}\right), 2.39$ (s, 3H, $\left.\mathrm{CH}_{3}\right), 2.38(\mathrm{~m}, 2 \mathrm{H}, 2 \times \mathrm{CH}), 1.35$ (br d, $J=6.0 \mathrm{~Hz}, 6 \mathrm{H}$, $\left.2 \mathrm{xCH}_{3}\right), 1.00\left(\mathrm{~d}, J=6.8 \mathrm{~Hz}, 6 \mathrm{H}, 2 \mathrm{xCH}_{3}\right) ;{ }^{13} \mathrm{C} \mathrm{NMR}\left(75 \mathrm{MHz}, \mathrm{CDCl}_{3}\right): \delta=154.6(\mathrm{ArC}), 150.5(\mathrm{ArC})$, $149.5(\mathrm{NCHN}), 144.7$ (ArC), 137.8 (ArC), 133.8 (ArC), $133.3(\mathrm{ArCH}), 133.0(\mathrm{ArCH}), 132.0(\mathrm{ArCH})$, 129.5 (2xArCH), 128.0 (2xArCH), 127.6 (ArC), 127.3 (ArC), 125.6 (2xArCH), 121.9 (ArC), 29.5 (2xCH), $24.7\left(2 \mathrm{xCH}_{3}\right), 22.6\left(2 \mathrm{xCH}_{3}\right), 18.2\left(\mathrm{CH}_{3}\right), 17.8\left(\mathrm{CH}_{3}\right), 13.5\left(\mathrm{CH}_{3}\right)$; IR $(\mathrm{ATR}): \tilde{v}\left[\mathrm{~cm}^{-1}\right]=2968$ (m), 2602 (m), 1605 (w), 1585 (w), 1526 (s), 1445 (m), 1368 (m), $1221(\mathrm{w}), 1059$ (m), 945 (w), 845 (m), $783(\mathrm{~m}), 752(\mathrm{~m}), 694(\mathrm{~m})$; ESI-MS (positive): $\mathrm{m} / \mathrm{z}=469\left(\mathrm{M}^{+}-\mathrm{Cl}, 100 \%\right)$; elemental analysis calcd (\%) for $\mathrm{C}_{29} \mathrm{H}_{33} \mathrm{~N}_{4} \mathrm{O}_{2} \mathrm{Cl} \bullet \mathrm{H}_{2} \mathrm{O}$ : C, 66.59; $\mathrm{H}, 6.74 ; \mathrm{N}, 10.71$; found: C, 66.51; H, 6.53; N, 10.69.

\section{4-(2,6-Diisopropyl)phenyl)-1-(2,6-dimethyl-3-nitrophenyl)-3-phenyl-1H-1,2,4-triazol-4-ium}

chloride (18). According to GP-3, the pre-formed 11b-Cl (2.6 g, $10.0 \mathrm{mmol}, 1.0$ equiv) was

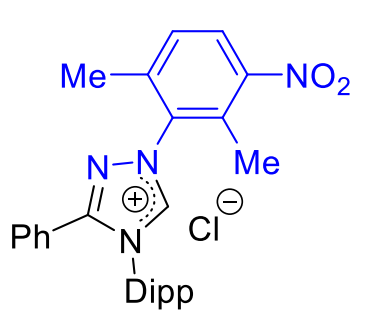

18 condensed with 10c (1.8 g, $10.0 \mathrm{mmol}, 1.0$ equiv) in the presence of NMM (1.3 $\mathrm{mL}, 12.0 \mathrm{mmol}, 1.2$ equiv) in THF $(30 \mathrm{~mL})$, followed by the reaction with $\mathrm{CH}(\mathrm{OEt})_{3}(5.0 \mathrm{~mL}, 30.0 \mathrm{mmol}, 3.0$ equiv) and $\mathrm{HCl} /$ dioxane $(4 \mathrm{M}, 3.8 \mathrm{~mL}, 15.0$ mmol, 1.5 equiv) in $\mathrm{PhCl}(5.0 \mathrm{~mL})$ at $110{ }^{\circ} \mathrm{C}$ for $1 \mathrm{~h}$. After several triturations 
with EtOAc, 18 was obtained as a beige solid (1.5 g, $3.0 \mathrm{mmol}, 30 \%)$ : mp 242-243 ${ }^{\circ} \mathrm{C} ;{ }^{1} \mathrm{H}$ NMR (300 $\mathrm{MHz}_{\mathrm{CDCl}}$ ): $\delta=14.03(\mathrm{~s}, 1 \mathrm{H}, \mathrm{NCHN}), 8.16(\mathrm{~d}, J=8.5 \mathrm{~Hz}, 1 \mathrm{H}, \mathrm{ArH}), 7.67(\mathrm{t}, J=7.8 \mathrm{~Hz}, 1 \mathrm{H}, \mathrm{ArH})$, $7.57(\mathrm{t}, J=7.2 \mathrm{~Hz}, 1 \mathrm{H}, \mathrm{ArH}), 7.54-7.34(\mathrm{~m}, 7 \mathrm{H}), 2.51\left(\mathrm{~s}, 3 \mathrm{H}, \mathrm{CH}_{3}\right), 2.47\left(\mathrm{~s}, 3 \mathrm{H}, \mathrm{CH}_{3}\right), 2.43(\mathrm{~m}, 2 \mathrm{H}$, 2xCHMe 2$), 1.39$ and 1.34 (2x br d, $\left.J=6.3 \mathrm{~Hz}, 6 \mathrm{H}, 2 \mathrm{xCH}_{3}\right), 1.03$ (br s, 6H, 2xCH$) ;{ }^{13} \mathrm{C}$ NMR $(75$ $\mathrm{MHz}, \mathrm{CDCl}_{3}$ ): $\delta=154.8(\mathrm{ArC}), 149.5(\mathrm{NCHN}), 148.4(\mathrm{ArC}), 144.7$ (ArC), $141.6(\mathrm{ArC}), 134.9(\mathrm{ArC})$, $133.4(\mathrm{ArCH}), 133.1(\mathrm{ArCH}), 131.4(\mathrm{ArC}), 129.7(\mathrm{ArCH}), 129.6(2 \times \mathrm{ArCH}), 128.0$ (2xArCH), 127.8 $(\mathrm{ArCH}), 127.3(\mathrm{ArC}), 125.6(2 \mathrm{xArCH}), 121.6(\mathrm{ArC}), 29.5(2 \mathrm{xCH}), 24.7\left(2 \mathrm{xCH}_{3}\right), 22.5\left(2 \mathrm{xCH}_{3}\right), 18.6$ $\left(\mathrm{CH}_{3}\right), 14.9\left(\mathrm{CH}_{3}\right)$; IR (ATR): $\tilde{v}\left[\mathrm{~cm}^{-1}\right]=2965(\mathrm{~m}), 2872(\mathrm{w}), 2581(\mathrm{~m}), 1601(\mathrm{w}), 1587(\mathrm{w}), 1549$ (m), 1522 (s), 1447 (m), 1342 (s), 1211 (m), 936 (m), 851 (m), 814 (m), 775 (m), 721 (s), 687 (s); ESIMS (positive): $m / z=455\left(\mathrm{M}^{+}-\mathrm{Cl}, 100 \%\right)$; elemental analysis calcd $(\%)$ for $\mathrm{C}_{28} \mathrm{H}_{31} \mathrm{~N}_{4} \mathrm{O}_{2} \mathrm{Cl}: \mathrm{C}, 68.49 ; \mathrm{H}$, 6.36; N, 11.41; found: C, 68.29; H, 6.08; N, 11.13.

\section{1-(2,6-Diethyl-3-nitrophenyl)-4-(2,6-diisopropyl)phenyl)-3-phenyl-1H-1,2,4-triazol-4-ium}

chloride (19). According to GP-3, the pre-formed 11b-Cl (2.6 g, $10.0 \mathrm{mmol}, 1.0$ equiv) was

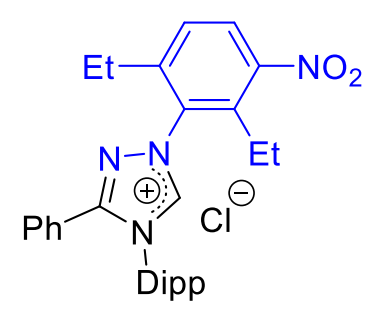

19 condensed with $\mathbf{1 0 d} \bullet \mathrm{HCl}(2.9 \mathrm{~g}, 11.8 \mathrm{mmol}, 1.2$ equiv) in the presence of NMM (1.3 mL, $12.0 \mathrm{mmol}, 1.2$ equiv) in THF $(50 \mathrm{~mL})$, followed by the reaction with $\mathrm{CH}(\mathrm{OEt})_{3}(5.0 \mathrm{~mL}, 30.0 \mathrm{mmol}, 3.0$ equiv) and $\mathrm{HCl} /$ dioxane (4 $\mathrm{M}, 2.5 \mathrm{~mL}, 10.0 \mathrm{mmol}, 1.0$ equiv) in $\mathrm{PhCl}(5.0 \mathrm{~mL})$ at $110{ }^{\circ} \mathrm{C}$ for $1 \mathrm{~h}$. After several triturations with EtOAc, 19 was obtained as a cream colored solid (1.3 g, $2.5 \mathrm{mmol}, 25 \%): \mathrm{mp} 235-236{ }^{\circ} \mathrm{C} ;{ }^{1} \mathrm{H} \mathrm{NMR}\left(300 \mathrm{MHz}, \mathrm{CDCl}_{3}\right): \delta=14.27$ $(\mathrm{s}, 1 \mathrm{H}, \mathrm{NCHN}), 8.15(\mathrm{~d}, J=8.6 \mathrm{~Hz}, 1 \mathrm{H}, \mathrm{ArH}), 7.65(\mathrm{t}, J=7.8 \mathrm{~Hz}, 1 \mathrm{H}), 7.60-$ $7.48(\mathrm{~m}, 2 \mathrm{H}), 7.48-7.33(\mathrm{~m}, 6 \mathrm{H}), 2.86-2.32\left(\mathrm{~m}, 6 \mathrm{H}, 2 \mathrm{xCH}_{2}\right.$ and $\left.2 \mathrm{xCH}\right), 1.42-1.24(\mathrm{~m}, 12 \mathrm{H}$, $\left.4 \mathrm{xCH}_{3}\right), 1.01\left(\mathrm{~d}, J=6.7 \mathrm{~Hz}, 6 \mathrm{H}, 2 \mathrm{xCH}_{3}\right) ;{ }^{13} \mathrm{C} \mathrm{NMR}\left(75 \mathrm{MHz}, \mathrm{CDCl}_{3}\right): \delta=154.4(\mathrm{ArC}), 150.3$ ( $\mathrm{NCHN}$ ), 148.2 (ArC), 147.2 (ArC), 144.7 (ArC), 136.7 (ArC), 134.2 (ArC), 133.4 (ArCH), 133.1 $(\mathrm{ArCH}), 129.6(2 \mathrm{xArCH}), 128.4(\mathrm{ArCH}), 128.1(3 \mathrm{xArCH}), 127.3(\mathrm{ArC}), 125.6(2 \times \mathrm{ArCH}), 121.8$ (ArC), $29.6(2 x C H), 25.1\left(\mathrm{CH}_{2}\right), 24.8\left(2 \mathrm{xCH}_{3}\right), 22.6\left(\mathrm{CH}_{3}\right), 22.5\left(\mathrm{CH}_{3}\right), 22.1\left(\mathrm{CH}_{2}\right), 14.8\left(\mathrm{CH}_{3}\right), 13.9$ $\left(\mathrm{CH}_{3}\right)$; IR (ATR): $\tilde{v}\left[\mathrm{~cm}^{-1}\right]=2970(\mathrm{~m}), 2872(\mathrm{w}), 2554(\mathrm{~m}), 2467(\mathrm{w}), 1587(\mathrm{w}), 1547(\mathrm{~m}), 1522(\mathrm{~s})$, $1466(\mathrm{~m}), 1354$ (m), 1059 (m), 818 (m), 777 (m), 723 (s), 691 (s); ESI-MS (positive): $m / z=483\left(\mathrm{M}^{+}-\right.$ $\mathrm{Cl}, 100 \%)$; elemental analysis calcd $(\%)$ for $\mathrm{C}_{30} \mathrm{H}_{35} \mathrm{~N}_{4} \mathrm{O}_{2} \mathrm{Cl}: \mathrm{C}, 69.42 ; \mathrm{H}, 6.80 ; \mathrm{N}, 10.79$; found: $\mathrm{C}$, 69.20; H, 6.61; N, 10.57 .

\section{Synthesis of 8-oxocitronellal (5) from citronellol}

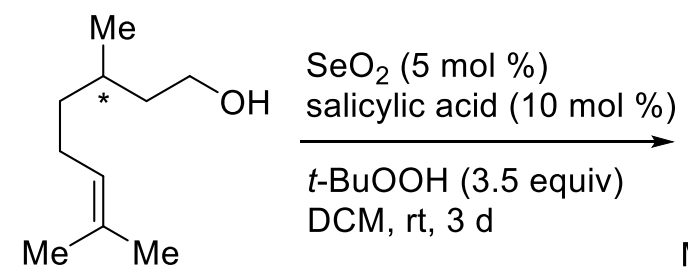

$(S)$ - or $(R)$-citronellol

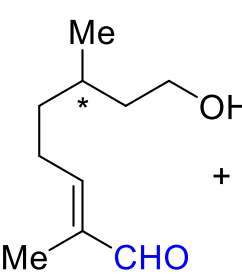

6

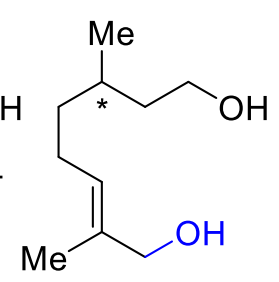

7

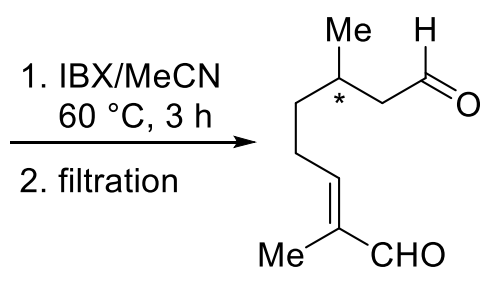

5

For the synthesis of $\mathbf{5}$, a modification of a procedure described by Hofferberth and co-workers was employed. ${ }^{4}(S)$-Citronellol (10 g, $64.0 \mathrm{mmol}, 1.0$ equiv) was added to a mixture of $\mathrm{SeO}_{2}(0.36 \mathrm{~g}$,

\footnotetext{
${ }^{4}$ Fischman, C. J.; Adler, S.; Hofferberth, J. E. J. Org. Chem. 2013, 78, 7318-7323.
} 
$3.3 \mathrm{mmol}, 5 \mathrm{~mol} \%)$, salicylic acid (0.92 g, $6.7 \mathrm{mmol}, 10 \mathrm{~mol} \%), t$-BuOOH (70\% in $\mathrm{H}_{2} \mathrm{O}, d=0.94,30$ $\mathrm{mL}, 219 \mathrm{mmol}, 3.4$ equiv), and DCM $(20 \mathrm{~mL})$. The reaction mixture was stirred at $\mathrm{rt}$ for $3 \mathrm{~d}$, diluted with toluene $(50 \mathrm{~mL})$ and evaporated on a rotary evaporator. The residue was diluted with $\mathrm{Et}_{2} \mathrm{O}(200$ $\mathrm{mL})$, washed with $10 \% \mathrm{NaOH}(6 \times 25 \mathrm{~mL})$ and brine, dried over anhyd $\mathrm{MgSO}_{4}$, and filtered. After removal of the solvent, the resulting pale yellow oil (10.8 g), containing a mixture of $(S)-\mathbf{6}$ and $(S)-7$, was purified by passing it though a short pad of silica gel. First, by eluting with EtOAc/cHex, 1:4, less polar impurities of $R_{\mathrm{f}}$ ca. 0.6 (EtOAc/Hex, 1:1) were removed. Then, using EtOAc/cHex, 3:1 as eluent, a mixture of $(S)-6$ and $(S)-7$ was obtained $\left[R_{\mathrm{f}}=0.32\right.$ and $0.13($ EtOAc/Hex, 1:1), $7.7 \mathrm{~g}, 45.0 \mathrm{mmol}$, $70 \%]$.

To this mixture of $(S)-6$ and $(S)-7$ (45.0 mmol, 1.0 equiv) in dry MeCN (100 mL) was added IBX (22.0 g, $78.6 \mathrm{mmol}, 1.8$ equiv). The suspension was stirred at $60{ }^{\circ} \mathrm{C}$ for $3 \mathrm{~h}$, cooled down to rt, and

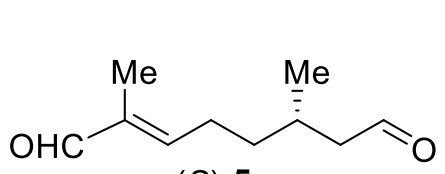

$(S)-5$ diluted with EtOAc $(100 \mathrm{~mL})$. The solid was filtered off with a glass frit and washed with EtOAc, affording IBA which can be oxidized to IBX in $86 \%$ yield with Oxone, following the procedure described by Santagostino and co-workers. ${ }^{5}$ The filtrate was concentrated on a rotary evaporator and purified by column chromatography on silica (EtOAc/cHex, 1:9) to give $(S)-5(6.2 \mathrm{~g}$, $36.9 \mathrm{mmol}, 58 \%)$ as a pale yellow oil: $R_{\mathrm{f}}=0.63($ EtOAc/Hex, $1: 1) ;{ }^{1} \mathrm{H}$ NMR $\left(300 \mathrm{MHz}, \mathrm{CDCl}_{3}\right): \delta=$ $9.78(\mathrm{t}, J=2.0 \mathrm{~Hz}, 1 \mathrm{H}, \mathrm{CHO}), 9.40(\mathrm{~s}, 1 \mathrm{H}, \mathrm{CHO}), 6.48(\mathrm{td}, J=7.3,1.3 \mathrm{~Hz}, 1 \mathrm{H}, \mathrm{C}=\mathrm{CH}), 2.40(\mathrm{~m}, 4 \mathrm{H}$, $\left.2 \mathrm{xCH}_{2}\right), 2.14(\mathrm{~m}, 1 \mathrm{H}, \mathrm{CH}), 1.75(\mathrm{~s}, 3 \mathrm{H}), 1.63-1.36\left(\mathrm{~m}, 2 \mathrm{H}, \mathrm{CH}_{2}\right), 1.03\left(\mathrm{~d}, J=6.7 \mathrm{~Hz}, 3 \mathrm{H}, \mathrm{CH}_{3}\right) ;{ }^{13} \mathrm{C}$ NMR (75 MHz, $\left.\mathrm{CDCl}_{3}\right): \delta=202.1(\mathrm{CHO}), 195.1(\mathrm{CHO}), 153.8(\mathrm{CH}), 50.8\left(\mathrm{CH}_{2}\right), 35.2\left(\mathrm{CH}_{2}\right), 27.7$ (CH), $26.4\left(\mathrm{CH}_{2}\right), 19.6\left(\mathrm{CH}_{3}\right), 9.2\left(\mathrm{CH}_{3}\right)$; IR (ATR): $\tilde{v}\left[\mathrm{~cm}^{-1}\right]=2959(\mathrm{~m}), 2922(\mathrm{~m}), 2716(\mathrm{w}), 1721$ (m), 1682 (s), 1643 (m), 1406 (m), 1381 (m), 1242 (m), 1067 (m), 1005 (m), 868 (w), 816 (w); GCMS: $t_{\mathrm{R}}(\operatorname{Std} 50)=11.0 \mathrm{~min}, \mathrm{~m} / z=168\left(\mathrm{M}^{+}\right.$, trace $), 139(100 \%), 124,109,97,79,69,55$.<smiles>CC(C=O)=CCCC(C)CC=O</smiles>

$(R)-5$

$(R)-5$ was prepared in the same manner as described for $(S)-5$ starting from $(R)$-citronellol $(0.97 \mathrm{~g}, 5.8 \mathrm{mmol})$, providing $(R)-5$ in $55 \%$ yield $(0.54 \mathrm{~g}, 3.2 \mathrm{mmol})$ as a pale yellow oil.

\section{One-step synthesis of nepetalactones (1a) from 5}

\subsection{Procedure for the oxidative NHC-catalyzed transformation of 5 to 1a}

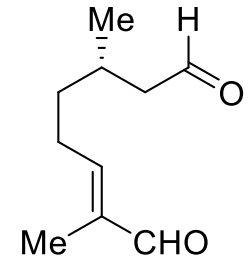

$(S)-5$

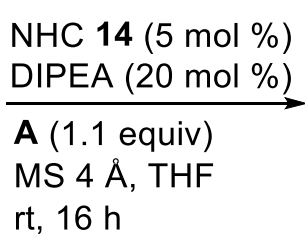

rt, $16 \mathrm{~h}$

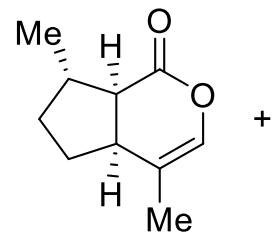

$(+)-1 a$

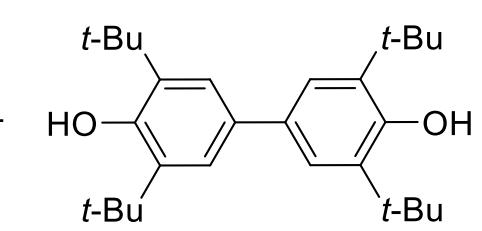

A- $\mathrm{H}_{2}$

(S)-5 (1.7 g, $10.0 \mathrm{mmol}, 1.0$ equiv) was added, under an Ar atmosphere, to a mixture of the triazolium salt 14 (0.24 g, $0.5 \mathrm{mmol}, 5 \mathrm{~mol} \%)$, the oxidant A (4.4 g, $10.8 \mathrm{mmol}, 1.1$ equiv), DIPEA (34.0 $\mu \mathrm{l}, 2.0 \mathrm{mmol}, 20 \mathrm{~mol} \%)$, and powdered MS $4 \AA$ ( $1.0 \mathrm{~g})$ in dry THF (25 mL). After stirring at rt for $16 \mathrm{~h}$ (to ensure full conversion), the reaction mixture was concentrated on a rotary evaporator. The

\footnotetext{
${ }^{5}$ Frigerio, M.; Santagostino, M.; Sputore, S. J. Org. Chem. 1999, 64, 4537-4538.
} 
residue was diluted with EtOAc/cHex (1:3) and filtered through a short pad of silica. The filtrate was evaporated under reduced pressure to dryness. $\mathrm{MeOH}(30 \mathrm{~mL})$ was added to the residue and the resulting suspension was stirred for $30 \mathrm{~min}$, followed by the addition of $\mathrm{H}_{2} \mathrm{O}(10 \mathrm{~mL})$ to precipitate more of $\mathbf{A}-\mathrm{H}_{2} / \mathbf{A}$. The solid was collected by suction filtration and washed with $\mathrm{MeOH} / \mathrm{H}_{2} \mathrm{O}(3: 1)$, affording $\mathbf{A}-\mathrm{H}_{2} / \mathbf{A}$ (4.3 g, quant) as a light brown solid.

The filtrate was evaporated under reduced pressure and extracted with DCM (addition of brine to aid good phase separation). The combined organic phases were dried over anhyd $\mathrm{Na}_{2} \mathrm{SO}_{4}$, filtered, and

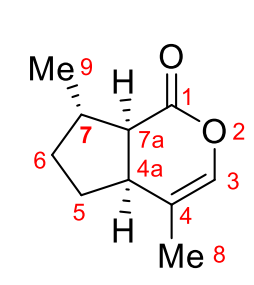

$(+)-1 \mathrm{a}$ concentrated by rotary evaporation. The resulting pale yellow oil was purified by the Kugelrohr distillation $\left(0.2\right.$ mbar/oven temperature $\left.100{ }^{\circ} \mathrm{C}\right)$ to give $(+)-\mathbf{1 a}(1.4 \mathrm{~g}, 8.4$ mmol, 84\%) as a colorless oil: $\mathrm{ds}>99 \%$ (GC on HP-5), ee $=97 \%$ (chiral GC on Lipodex E), $[\alpha]^{20}{ }_{\mathrm{D}}+21.9$ (c $\left.0.25, \mathrm{Et}_{2} \mathrm{O}\right)\left[\right.$ lit. $^{6}+20.6$ (c $0.24, \mathrm{Et}_{2} \mathrm{O} ;>95 \%$ ee $) ;{ }^{1} \mathrm{H}$ NMR (300 MHz, $\left.\mathrm{CDCl}_{3}\right): \delta=6.17(\mathrm{~m}, 1 \mathrm{H}, \mathrm{H} 3), 2.76$ (q, J = 7.7 Hz, 1H, H4a), 2.55 - 2.26 (m, 2H, H7a+H7), 2.04 (m, 1H, H5), 1.90 (m, 1H, H6), 1.63 (m, 3H, H8), 1.56 (m, 1H, H5), $1.23(\mathrm{~m}, 1 \mathrm{H}, \mathrm{H} 6), 1.20(\mathrm{~d}, J=6.5 \mathrm{~Hz}, 3 \mathrm{H}, \mathrm{H} 9) ;{ }^{13} \mathrm{C} \mathrm{NMR}\left(75 \mathrm{MHz}, \mathrm{CDCl}_{3}\right): \delta=170.9$ $(\mathrm{C}=\mathrm{O}), 133.5(\mathrm{CH}), 115.3\left(\mathrm{C}_{\mathrm{q}}\right), 49.3(\mathrm{CH}), 40.7(\mathrm{CH}), 39.7(\mathrm{CH}), 33.0\left(\mathrm{CH}_{2}\right), 30.9\left(\mathrm{CH}_{2}\right), 20.2\left(\mathrm{CH}_{3}\right)$, $15.5\left(\mathrm{CH}_{3}\right)$; IR (ATR): $\tilde{v}\left[\mathrm{~cm}^{-1}\right]=2957(\mathrm{~m}), 2870(\mathrm{w}), 1746(\mathrm{~s}), 1686(\mathrm{~m}), 1454(\mathrm{w}), 1337(\mathrm{~m}), 1198$ $(\mathrm{m}), 1125(\mathrm{~s}), 1028(\mathrm{~m}), 878(\mathrm{~m}), 826(\mathrm{w})$; GC-MS: $t_{\mathrm{R}}(\operatorname{Std} 50)=10.7 \mathrm{~min}, \mathrm{~m} / z=166\left(\mathrm{M}^{+}, 100 \%\right)$, $151,138,123,109,95,81,67,53$.

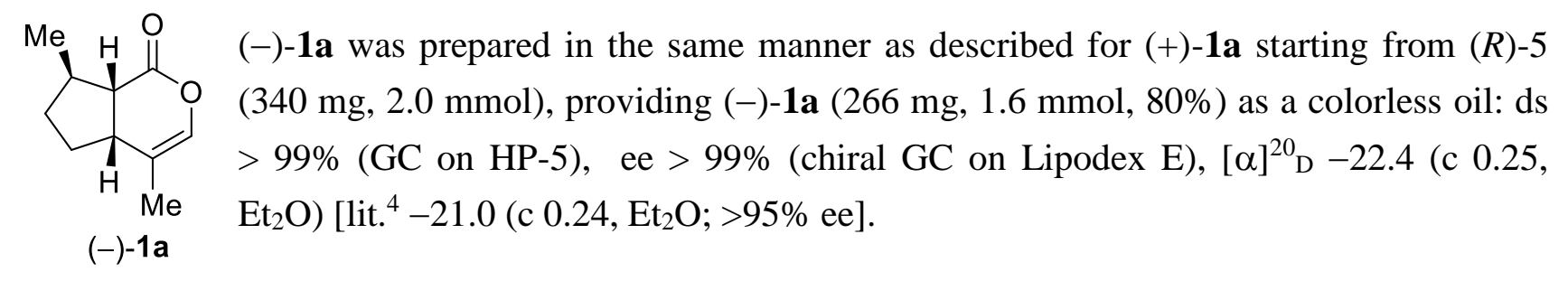

\subsection{Procedure for the oxidation of recovered $\mathrm{A}-\mathrm{H}_{2}$ to $\mathrm{A}$}
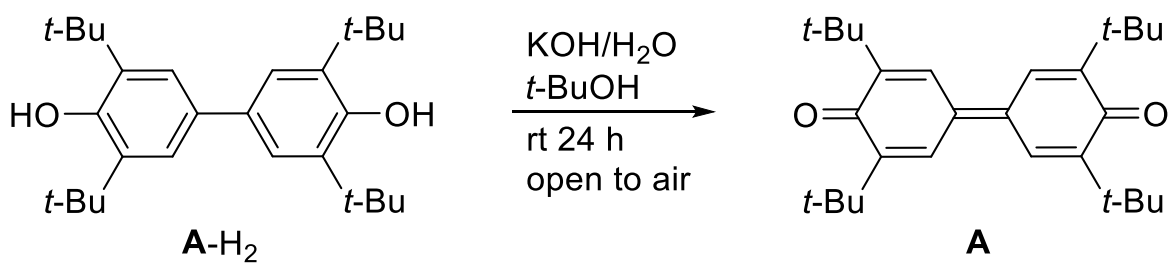

A

For the recycling of $\mathbf{A}-\mathrm{H}_{2}$ to $\mathbf{A}$, a modification of the literature known synthesis of $\mathbf{A}$ from 2,6-ditert-butylphenol was applied. ${ }^{7}$ A $250 \mathrm{~mL}$ Erlenmeyer flask was charged with $\mathbf{A}-\mathrm{H}_{2} / \mathbf{A}(13.5 \mathrm{~g}, 32.9$ mmol, 1.0 equiv, recovered from the oxidative NHC-catalyzed transformation of $\mathbf{5}$ to $\mathbf{1}), t$ - $\mathrm{BuOH}$ (120 $\mathrm{mL}$ ) and an aqueous solution of $\mathrm{KOH}\left(6.5 \mathrm{~g}, 115.9 \mathrm{mmol}, 3.5\right.$ equiv) in $\mathrm{H}_{2} \mathrm{O}$ (15 mL). The brownyellow suspension was stirred vigorously open to air at $\mathrm{rt}$ for $24 \mathrm{~h}$, resulting in a red-brown suspension. $\mathrm{H}_{2} \mathrm{O}(30 \mathrm{~mL})$ was added and stirring of the mixture was continued for $10 \mathrm{~min}$. The solid was collected by suction filtration and washed with water to give the product as a red-brown powder $(12.9 \mathrm{~g}, 96 \%)$. Further purification by recrystallization from toluene/EtOH (1:2) furnished $\mathbf{A}(12.1 \mathrm{~g}$, $29.6 \mathrm{mmol}, 90 \%$ ) as red-brown crystals with a purple reflex; 97\% purity, contaminated with $3 \% \mathrm{~A}-\mathrm{H}_{2}$

\footnotetext{
${ }^{6}$ Sakurai, K.; Ikeda, K.; Mori, K. Agric. Biol. Chem. 1988, 52, 2369-2371.

${ }^{7}$ Carrera, M.; de la Viuda, M.; Guijarro, A. Synlett 2016, 27, 2783-2787.
} 
(by NMR); mp 241-242 ${ }^{\circ} \mathrm{C}$ [lit. ${ }^{3} 243.1-244{ }^{\circ} \mathrm{C}$ (corrected)]; ${ }^{1} \mathrm{H}$ NMR $\left(300 \mathrm{MHz}, \mathrm{CDCl}_{3}\right): \delta=7.71$ (s, $4 \mathrm{H}, \mathrm{ArH}), 1.37\left(\mathrm{~s}, 36 \mathrm{H}, 12 \mathrm{xCH}_{3}\right) ;{ }^{13} \mathrm{C} \mathrm{NMR}\left(75 \mathrm{MHz}, \mathrm{CDCl}_{3}\right): \delta=186.4(\mathrm{C}=\mathrm{O}), 150.4\left(\mathrm{C}_{\mathrm{q}}\right), 136.1$ $\left.\left(\mathrm{C}_{\mathrm{q}}\right)\right), 126.0(\mathrm{ArCH}), 36.0\left(\mathrm{CMe}_{3}\right), 29.6\left(\mathrm{CH}_{3}\right)$; IR (ATR): $\tilde{v}\left[\mathrm{~cm}^{-1}\right]=3208(\mathrm{w}), 2957(\mathrm{~m}), 2907(\mathrm{~m})$, $2864(\mathrm{~m}), 1638$ (w), 1601 (s), 1566 (m), 1481 (m), 1456 (m), 1360 (s), 1260 (s), 1088 (s), 1040 (s), 934 (m), 897 (s), 881 (s), 843 (m), 818 (m), 797 (m), 747 (w).

\section{HPL chromatograms for the determination of the enantiomeric composition of citronellol}

The enantiomeric composition of citronellol was determined from the corresponding benzoyl ester. A small vial was charged with citronellol $(23.4 \mathrm{mg}, 0.15 \mathrm{mmol}, 1.0$ equiv) and pyridine (20.0 $\mu \mathrm{L}, 0.25$ mmol, 1.7 equiv). Benzoyl chloride $(20.0 \mu \mathrm{L}, 0.17 \mathrm{mmol}, 1.1$ equiv) was added and the resulting white suspension was stirred at rt for $30 \mathrm{~min}$. The reaction was quenched by adding $1 \mathrm{~N} \mathrm{HCl}$ and diluted with hexanes. Aliquots of the organic phase were withdrawn, filtered through a plug of silica gel and washed with hexanes for HPLC analysis. The NMR data of the analyzed 3,7-dimethyloct-6en-1-yl benzoate were identical to the literature. ${ }^{8}$

HPL chromatograms were acquired on a VWR Hitachi Chromaster HPLC equipped with Chiracel OD-H column. Conditions: solvent, $0.5 \%$ 2-propanol in hexanes; flow rate, $0.5 \mathrm{~mL} / \mathrm{min}$; oven temp, $18.0{ }^{\circ} \mathrm{C}$; detector, UV @ $230 \mathrm{~nm}$; $t_{\mathrm{R}}$ of $(R)$-enantiomer $=11.6 \mathrm{~min}$ and $t_{\mathrm{R}}$ of $(S)$-enantiomer $=12.4$ $\min$.

$(S)$-3,7-dimethyloct-6-en-1-yl benzoate derived from $(S)$-citronellol was $97 \%$ ee.

$(R)$-3,7-dimethyloct-6-en-1-yl benzoate derived from $(R)$-citronellol was $>99 \%$ ee.

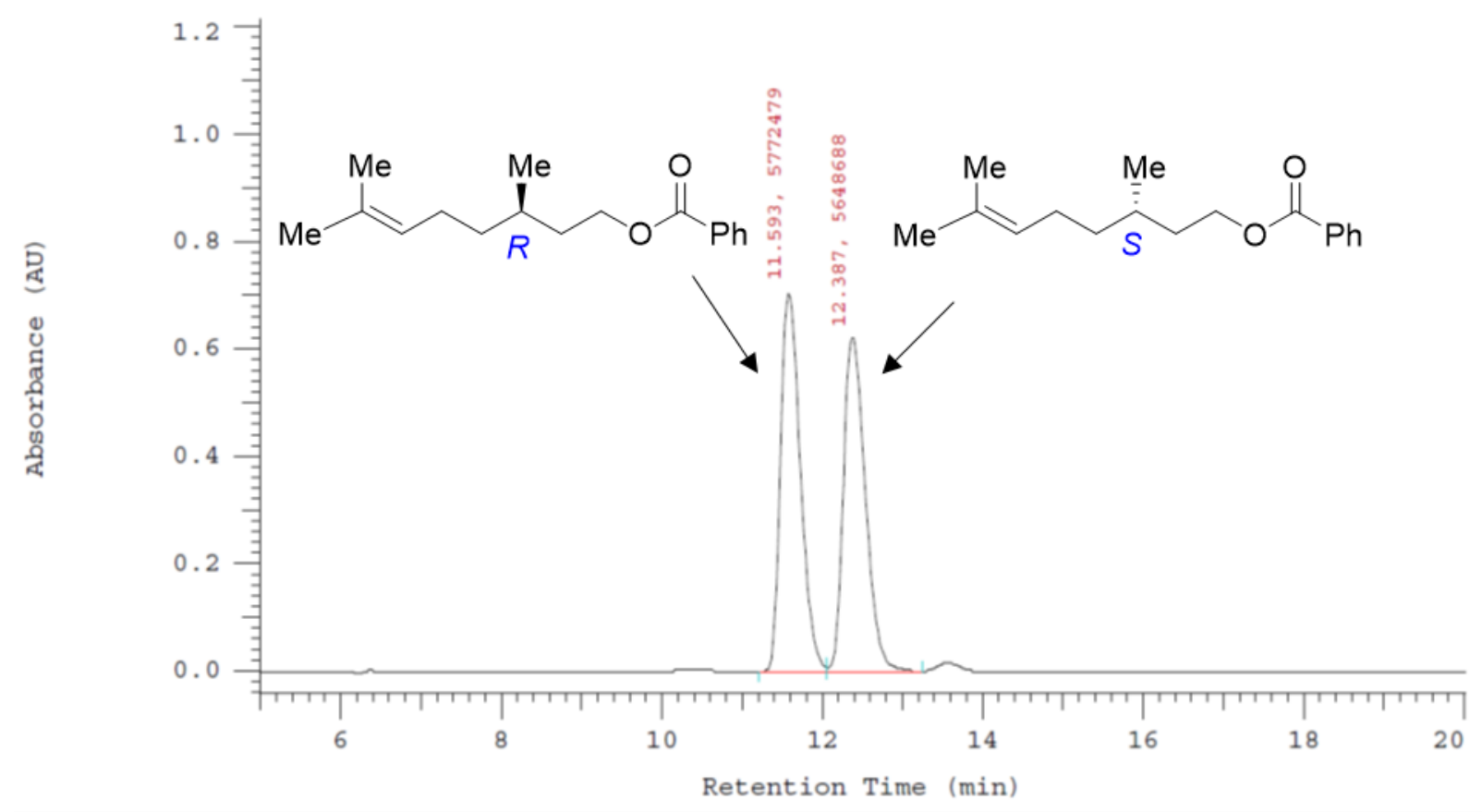

Figure S1. HPL chromatogram of rac-3,7-dimethyloct-6-en-1-yl benzoate.

${ }^{8}$ Ye, D.; Liu, Z.; Chen, H.; Sessler, J. L.; Lei, C. Org. Lett. 2019, 21, 6888-6892. 

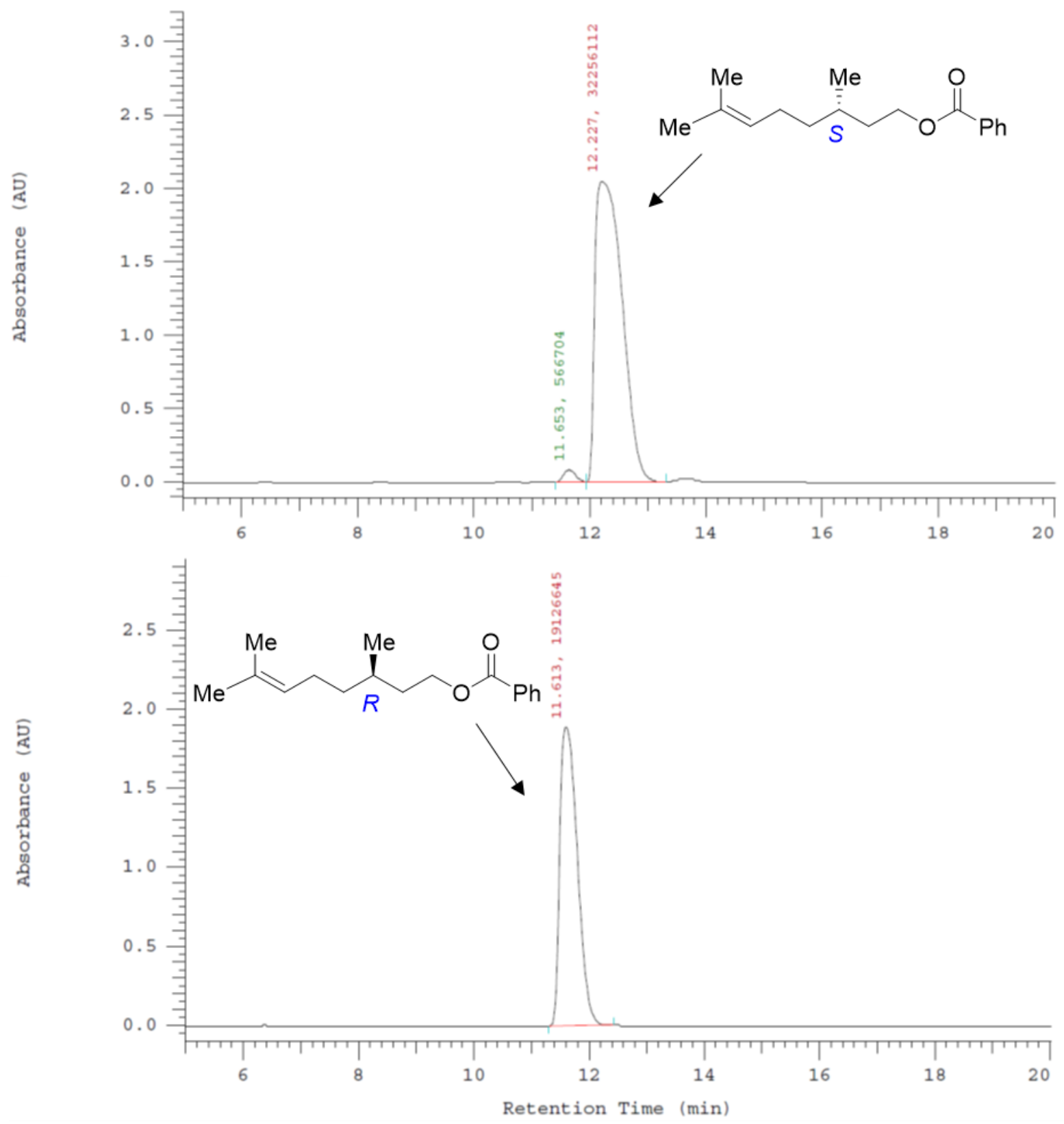

Figure S2. HPL chromatograms of $(S)$ - and $(R)$-3,7-dimethyloct-6-en-1-yl benzoate derived from commercial $(S)$ - and $(R)$-citronellol, respectively.

\section{Gas chromatograms for the determination of the enantiomeric composition of} $1 \mathrm{a}$

Column: Lipodex-E; conditions: $80{ }^{\circ} \mathrm{C}, 2 \mathrm{~min} ; 5{ }^{\circ} \mathrm{C} / \mathrm{min}$ to $120{ }^{\circ} \mathrm{C}, 5 \mathrm{~min} ; 1^{\circ} \mathrm{C} / \mathrm{min}$ to $135{ }^{\circ} \mathrm{C}, 5 \mathrm{~min}$; $10{ }^{\circ} \mathrm{C} / \mathrm{min}$ to $190{ }^{\circ} \mathrm{C}, 10 \mathrm{~min}$; flow $\left(\mathrm{N}_{2}\right), 1.0 \mathrm{~mL} / \mathrm{min} ; t_{\mathrm{R}}$ of $(+)-\mathbf{1 a}=21.9 \mathrm{~min}$ and $t_{\mathrm{R}}$ of $(+)-\mathbf{1 a}=25.5$ $\min$. 


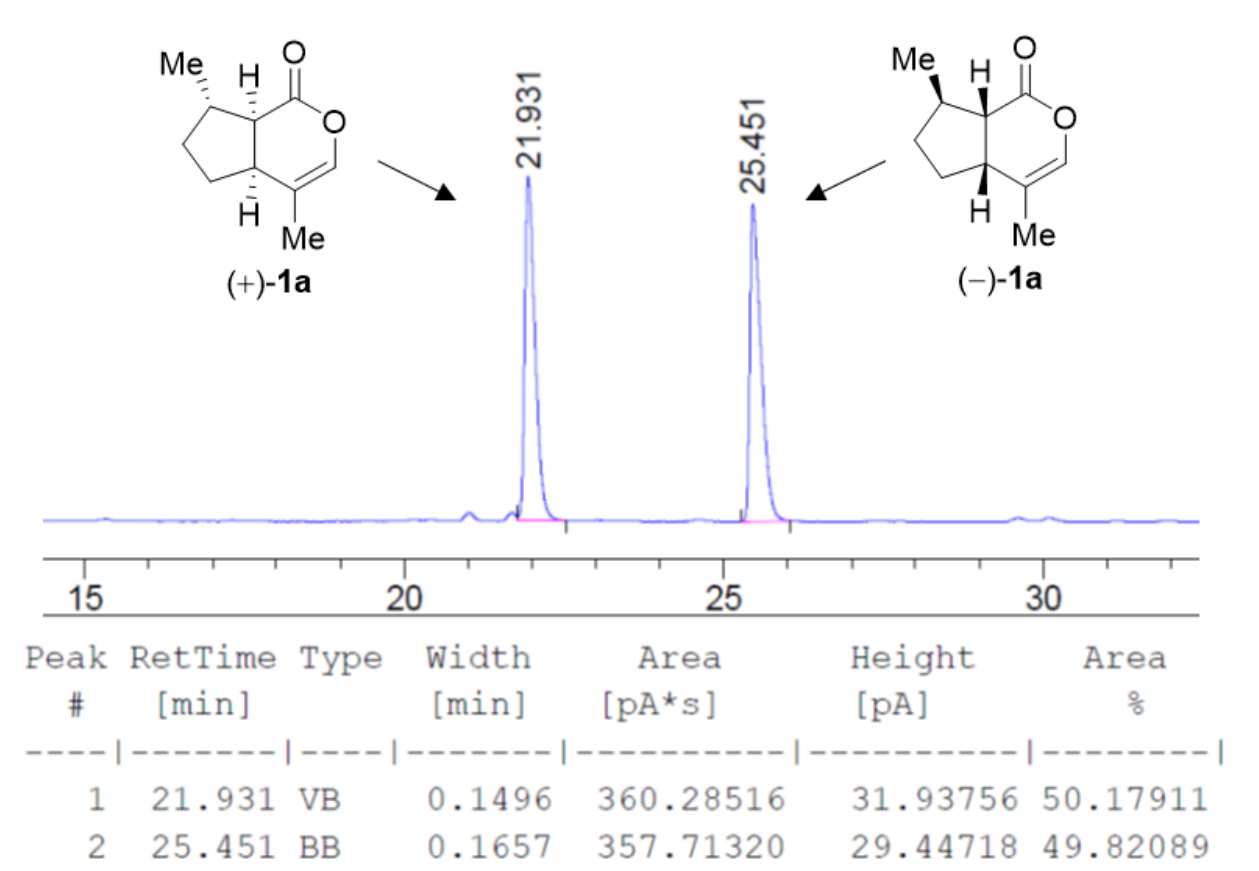

Figure S3. Gas chromatogram of $r a c-1 a$.

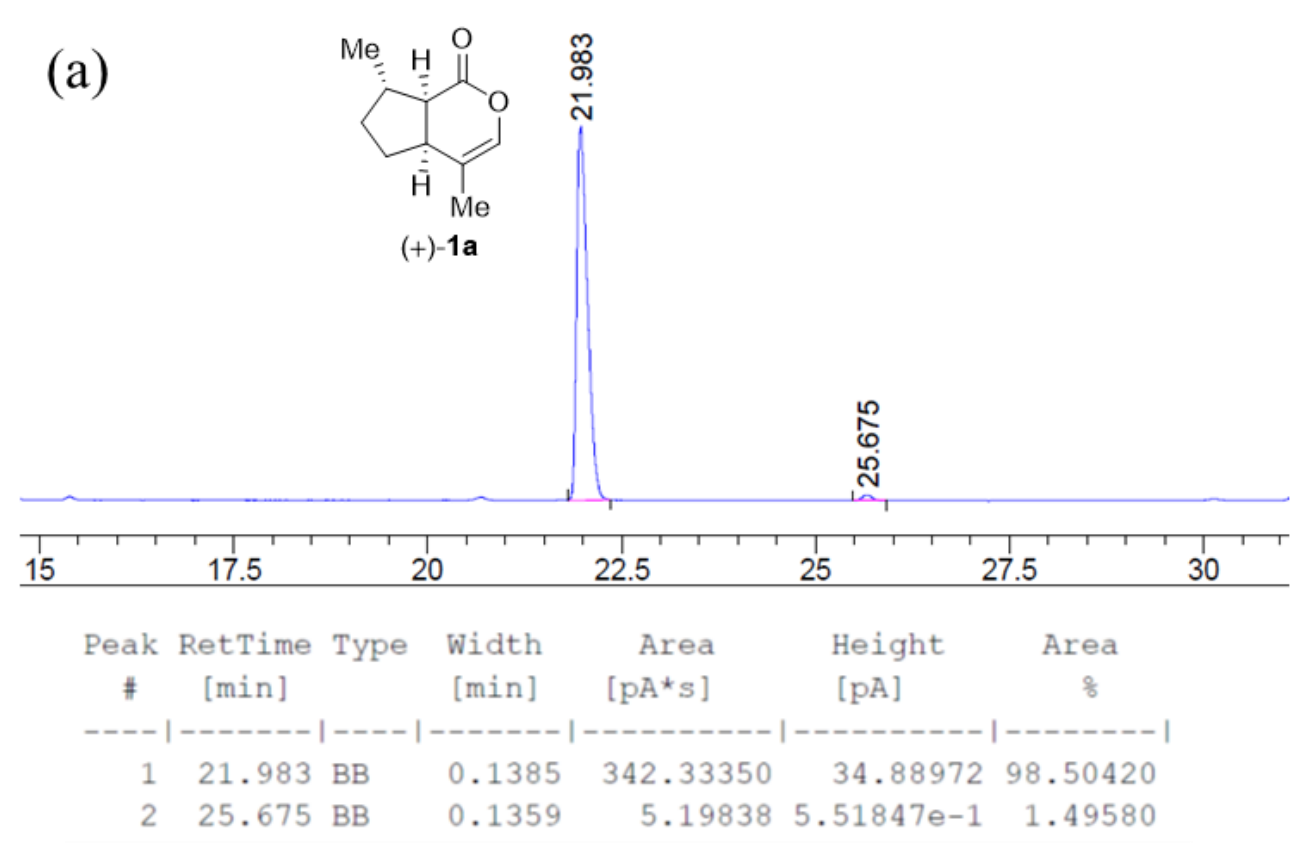

(b)

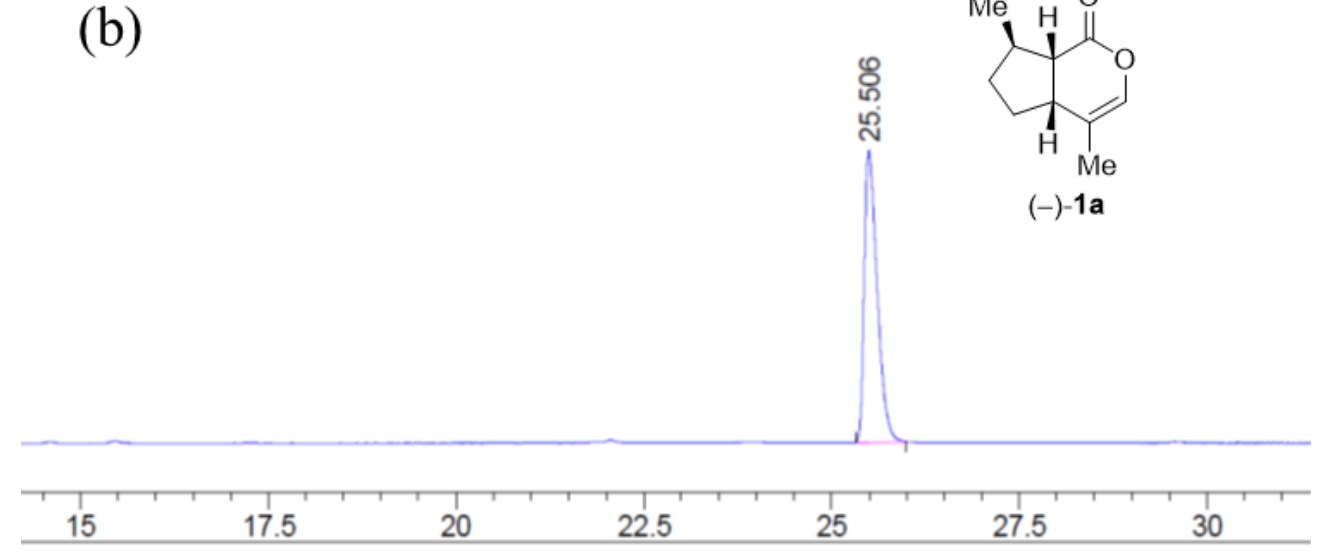

Figure S4. Gas chromatograms of (+)-1a (a) and (-)-1a (b). 
8. NMR spectra<smiles>Cc1c(N)c([N+](=O)[O-])c([N+](=O)[O-])c([N+](=O)[O-])c1[N+](=O)[O-]</smiles>
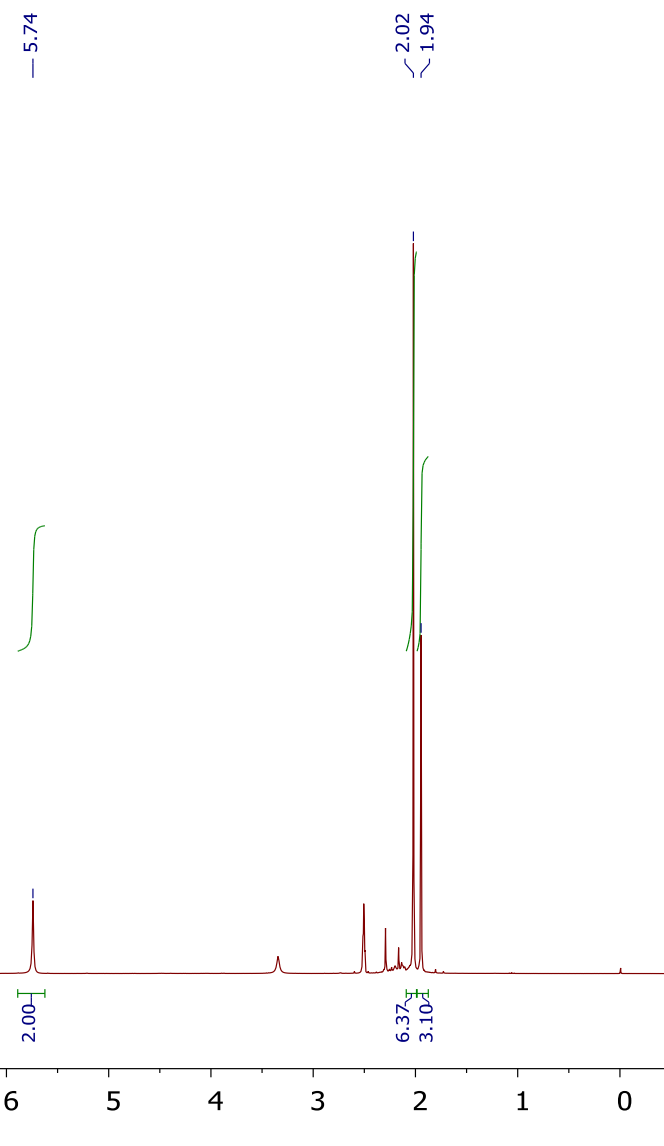

Figure S5. ${ }^{1} \mathrm{H}$ NMR $\left(300 \mathrm{MHz}, \mathrm{DMSO}-d_{6}\right)$ of 9a

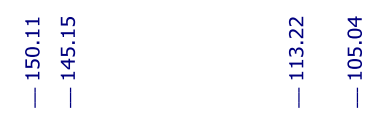<smiles>Cc1c(N)c([N+](=O)[O-])c([N+](=O)[O-])c([N+](=O)[O-])c1[N+](=O)[O-]</smiles>

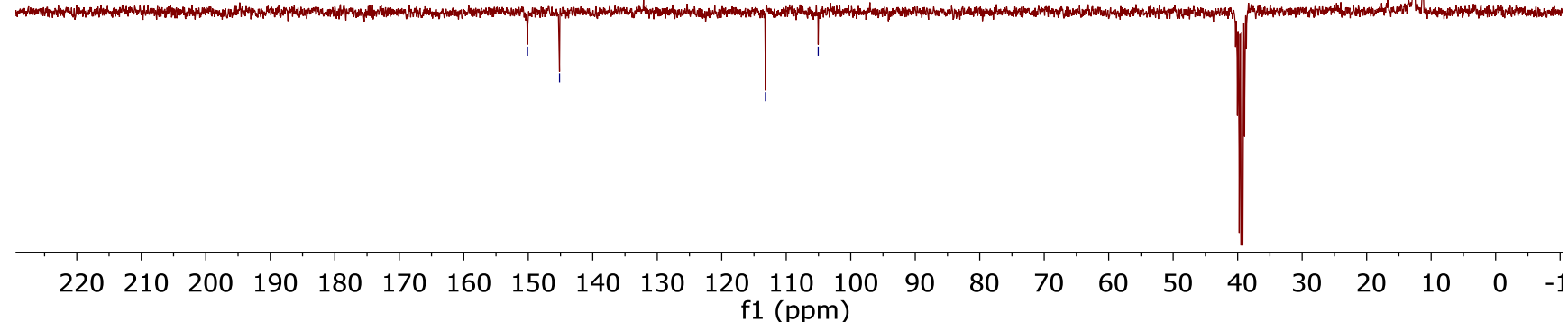

Figure S6. ${ }^{13} \mathrm{C}$ NMR (75 MHz, APT, DMSO- $\left.d_{6}\right)$ of 9a 


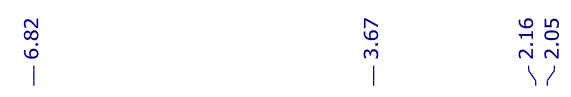<smiles>Cc1cc([N+](=O)[O-])c([N+](=O)[O-])c([N+](=O)[O-])c1N</smiles>

$9 b$

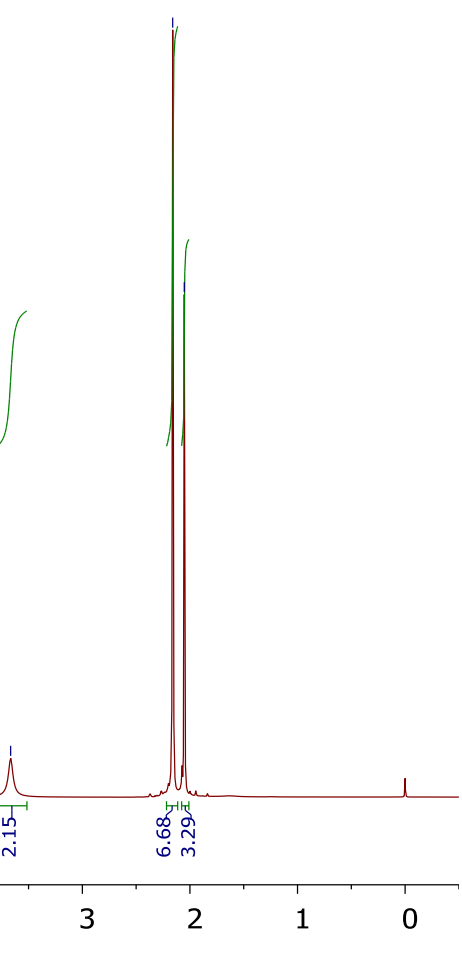

Figure S7. ${ }^{1} \mathrm{H} \mathrm{NMR}\left(300 \mathrm{MHz}, \mathrm{CDCl}_{3}\right)$ of $\mathbf{9 b}$

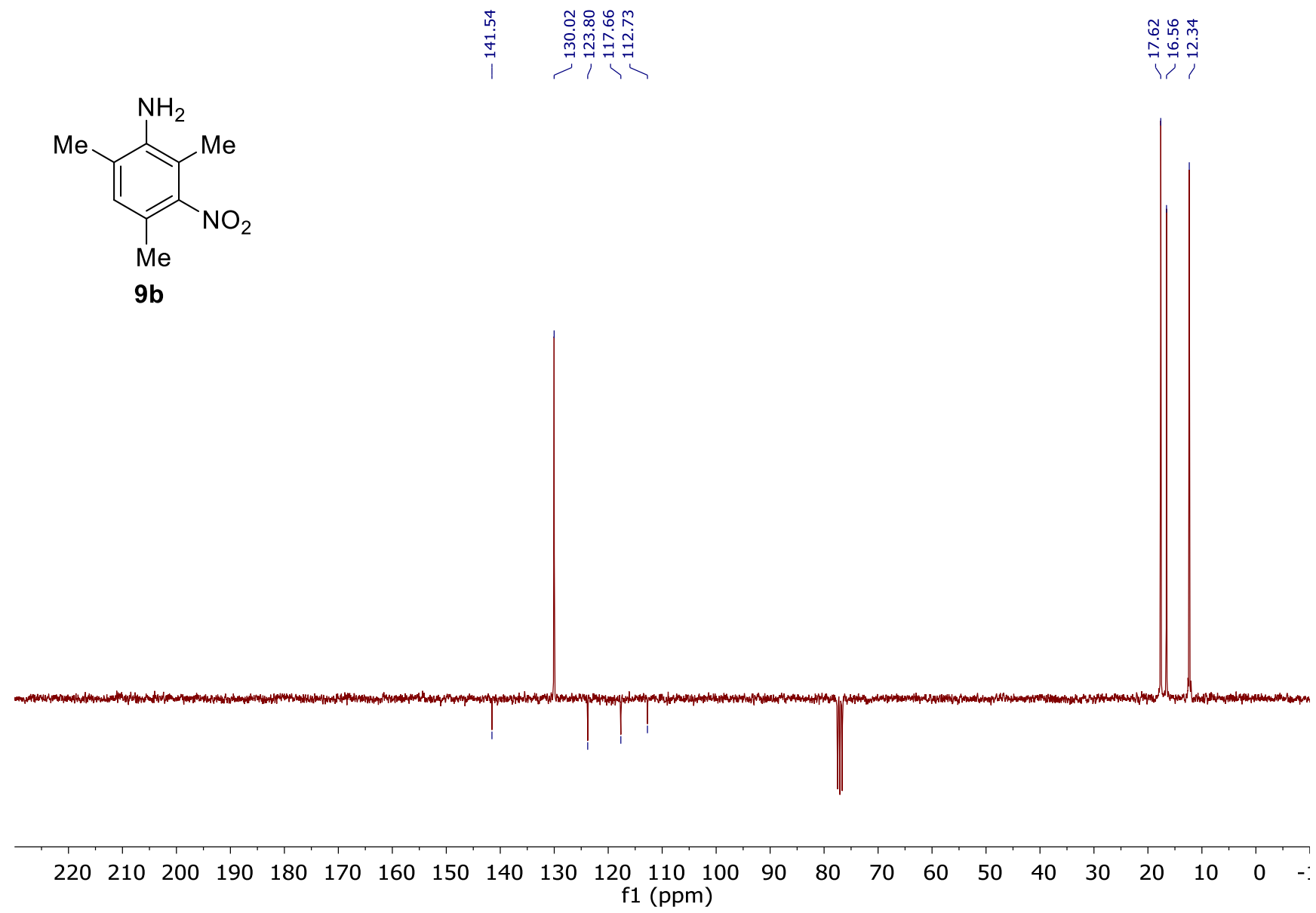

Figure S8. ${ }^{13} \mathrm{C}$ NMR $\left(75 \mathrm{MHz}, \mathrm{APT}, \mathrm{CDCl}_{3}\right)$ of $\mathbf{9 b}$ 


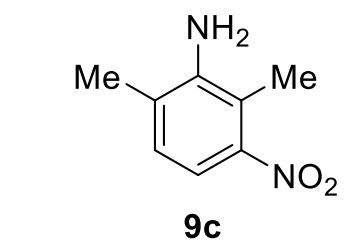

\section{鋘资跑}

ถูก

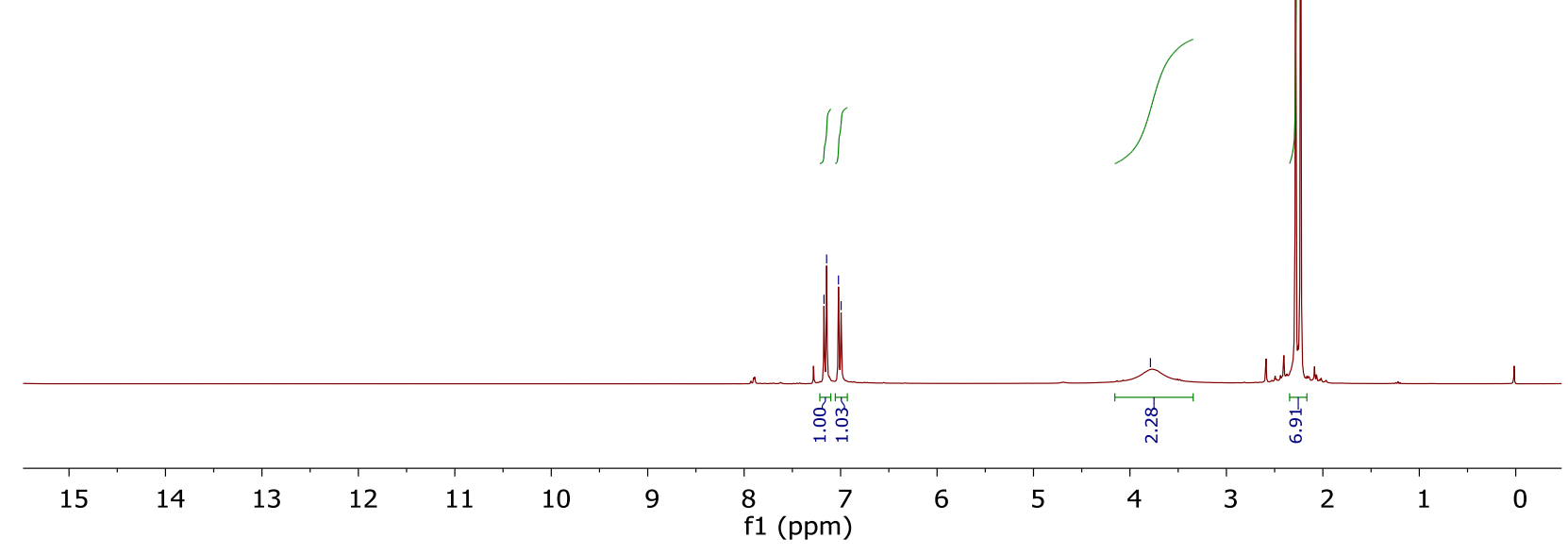

Figure S9. ${ }^{1} \mathrm{H} \mathrm{NMR}\left(300 \mathrm{MHz}, \mathrm{CDCl}_{3}\right)$ of $9 \mathrm{c}$
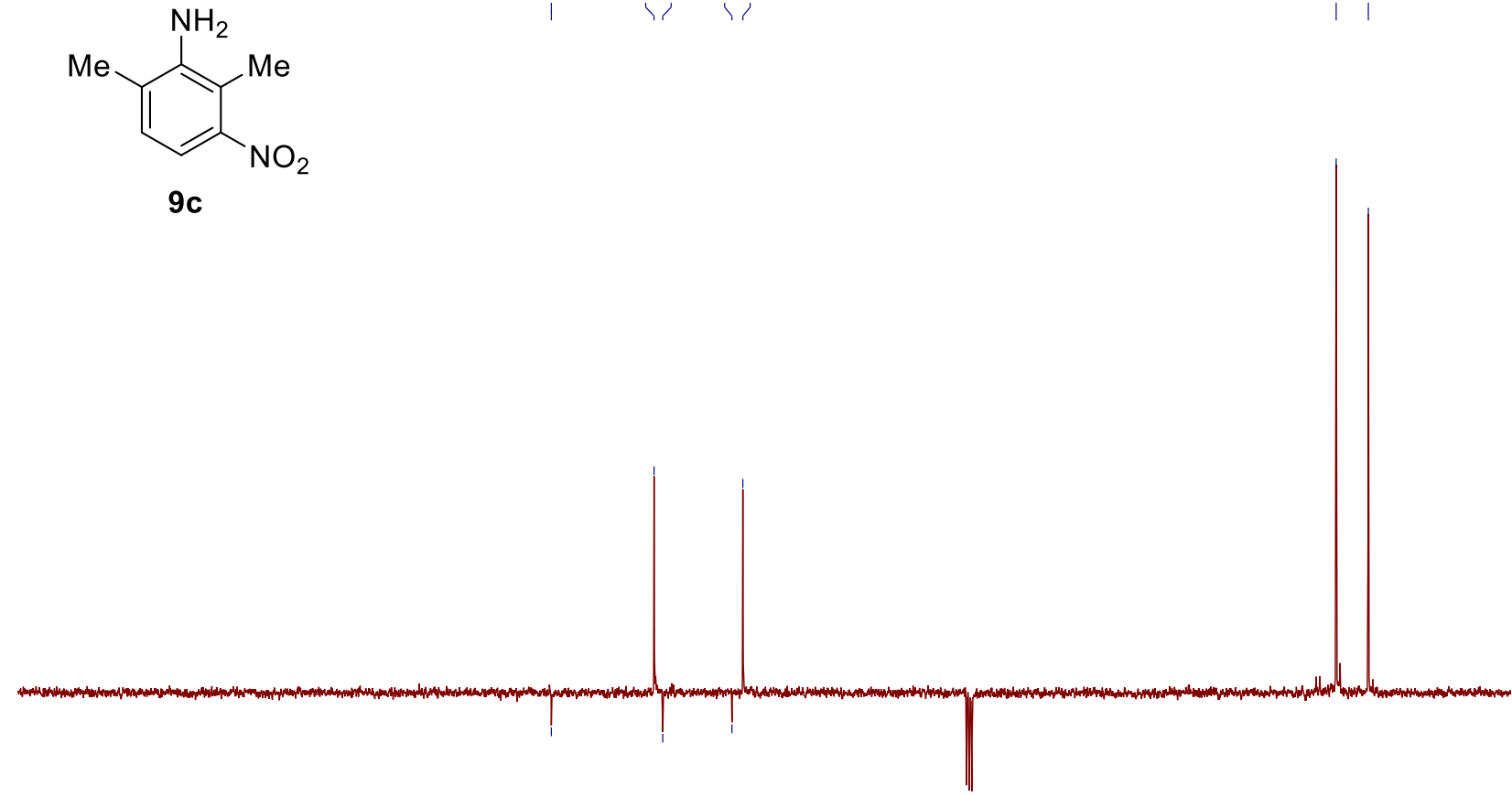

$\begin{array}{llllllllllllllllllllllll}220 & 210 & 200 & 190 & 180 & 170 & 160 & 150 & 140 & 130 & 120 & 110 & 100 & 90 & 80 & 70 & 60 & 50 & 40 & 30 & 20 & 10 & 0 & -\end{array}$

Figure S10. ${ }^{13} \mathrm{C} \mathrm{NMR}\left(75 \mathrm{MHz}, \mathrm{APT}, \mathrm{CDCl}_{3}\right)$ of $9 \mathrm{c}$ 
<smiles>CCc1ccc([N+](=O)[O-])c(CC)c1N</smiles>

9d

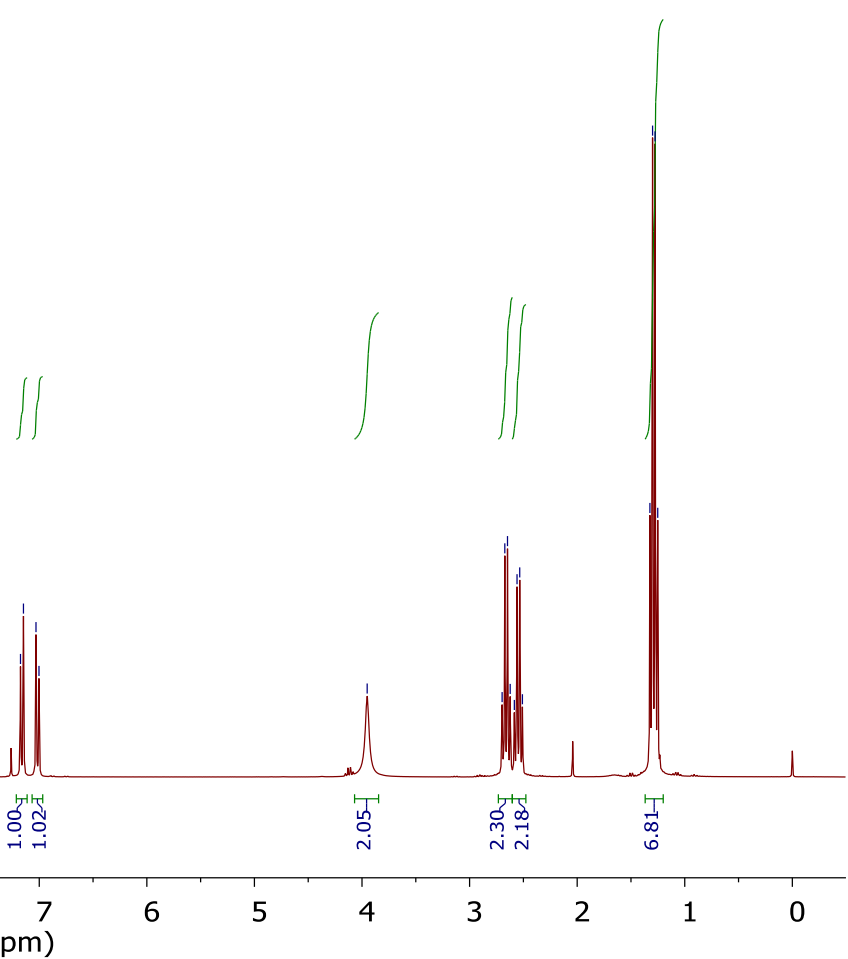

Figure S11. ${ }^{1} \mathrm{H}$ NMR $\left(300 \mathrm{MHz}, \mathrm{CDCl}_{3}\right)$ of 9d

$\begin{array}{llllllll}15 & 14 & 13 & 12 & 11 & 10 & 9 & 8 \\ & & & & & & \end{array}$<smiles>CCc1ccc([N+](=O)[O-])c(CC)c1N</smiles>

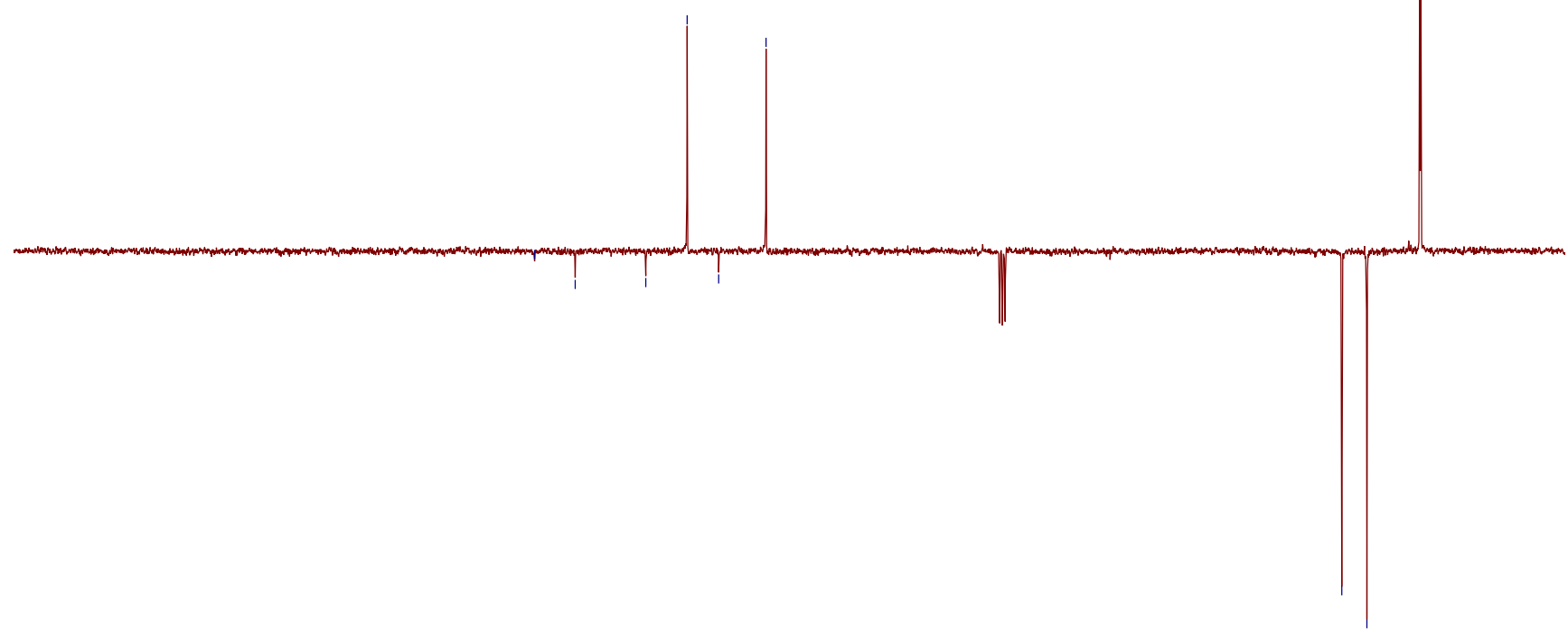

$\begin{array}{lllllllllllllllllllllll}220 & 210 & 200 & 190 & 180 & 170 & 160 & 150 & 140 & 130 & 120 \\ \mathrm{f} 1 & 110 & 100 & 90 & 80 & 70 & 60 & 50 & 40 & 30 & 20 & 10 & 0 & -\end{array}$

Figure S12. ${ }^{13} \mathrm{C}$ NMR $\left(75 \mathrm{MHz}, \mathrm{APT}, \mathrm{CDCl}_{3}\right)$ of $\mathbf{9 d}$ 
<smiles>Cc1c(C)c([N+](=O)[O-])c(C)c([N+](=O)[O-])c1NN</smiles>

$10 \mathrm{a} \cdot \mathrm{HCl}$
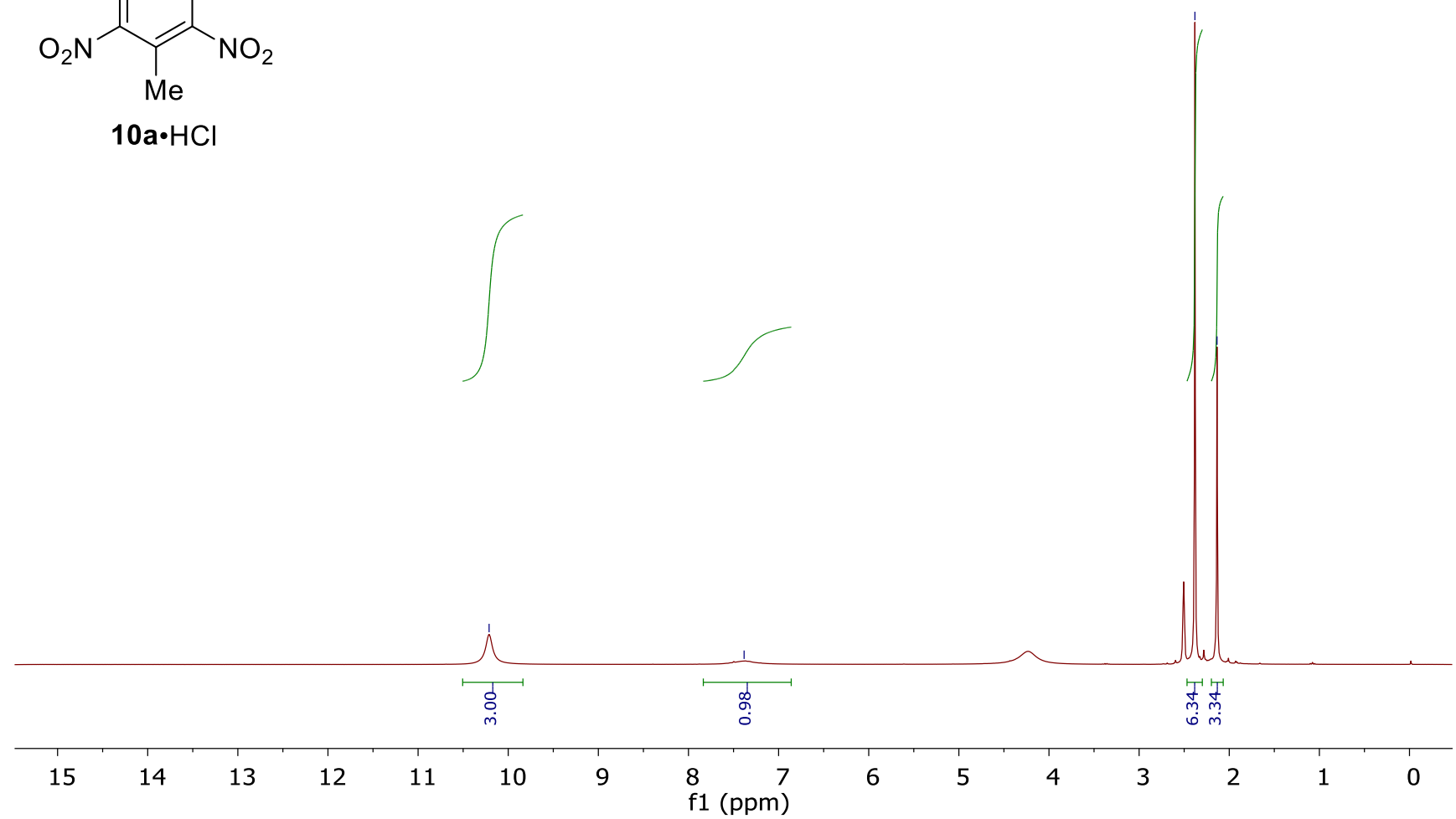

Figure S13. ${ }^{1} \mathrm{H}$ NMR (300 MHz, DMSO- $\left.d_{6}\right)$ of $\mathbf{1 0 a} \cdot \mathrm{HCl}$<smiles>Cc1c(NN)c([N+](=O)[O-])c([N+](=O)[O-])c([N+](=O)[O-])c1[N+](=O)[O-]</smiles>

$10 \mathrm{a} \cdot \mathrm{HCl}$

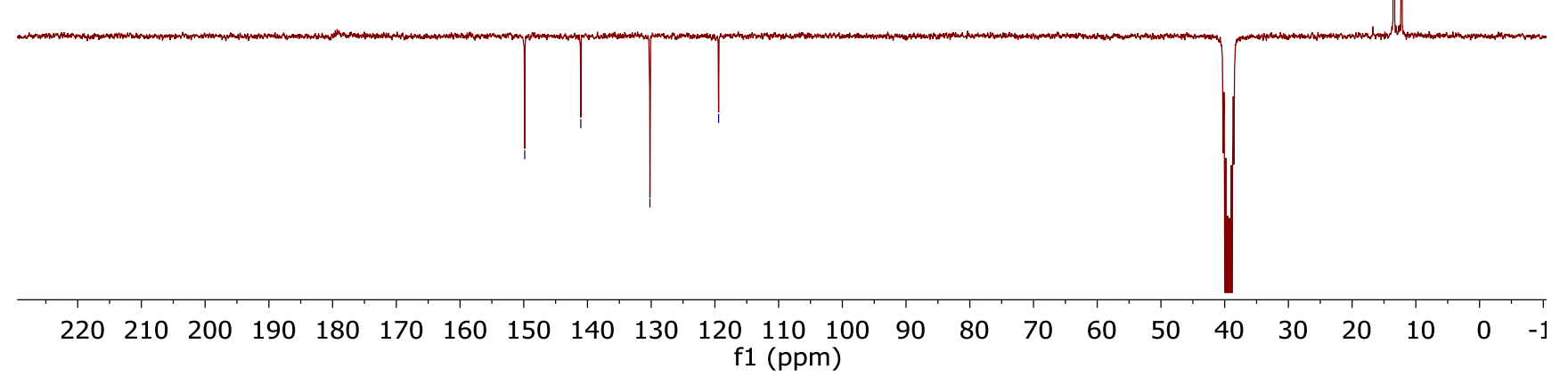

Figure S14. ${ }^{13} \mathrm{C}$ NMR $\left(75 \mathrm{MHz}, \mathrm{APT}, \mathrm{DMSO}-d_{6}\right)$ of $\mathbf{1 0 a} \cdot \mathrm{HCl}$ 
<smiles>Cc1c(NN)c([N+](=O)[O-])c([N+](=O)[O-])c([N+](=O)[O-])c1[N+](=O)[O-]</smiles>

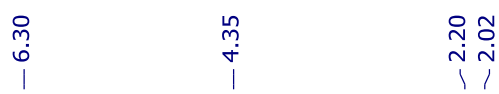

$10 \mathrm{a}$

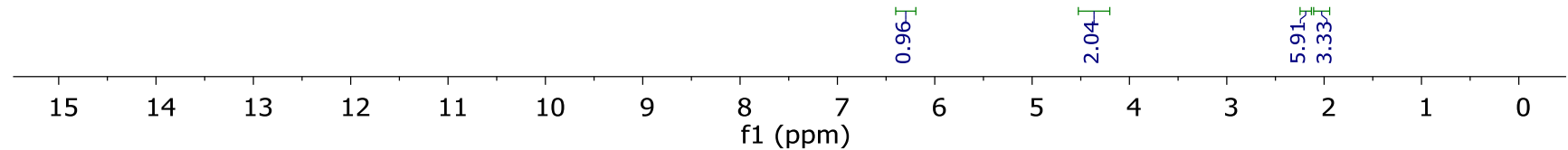

Figure S15. ${ }^{1} \mathrm{H}$ NMR (300 MHz, DMSO- $\left.d_{6}\right)$ of 10a<smiles>Cc1c(NN)c([N+](=O)[O-])c([N+](=O)[O-])c([N+](=O)[O-])c1[N+](=O)[O-]</smiles>

$10 \mathrm{a}$

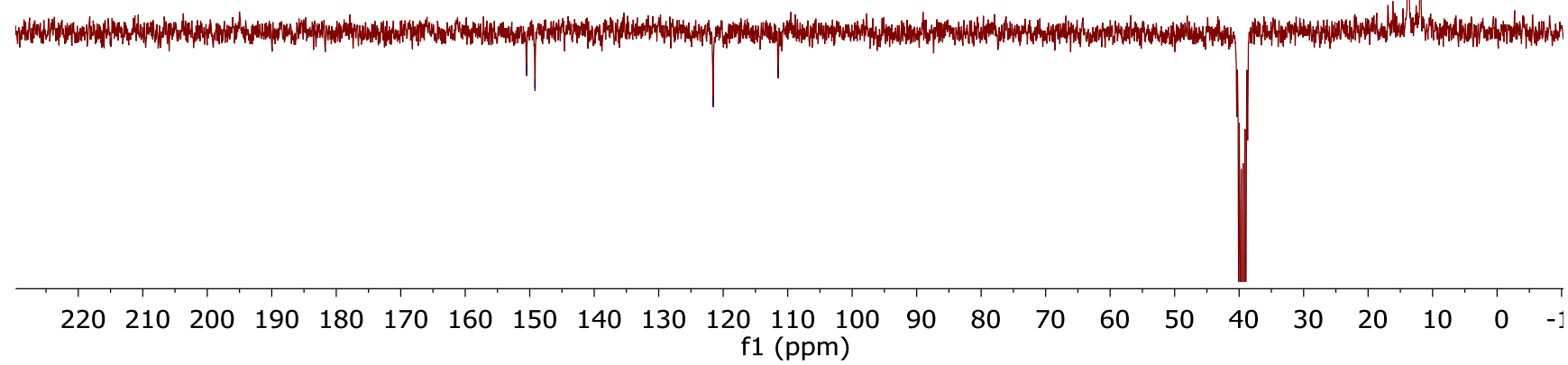

Figure S16. ${ }^{13} \mathrm{C}$ NMR (75 MHz, APT, DMSO- $\left.d_{6}\right)$ of 10a 


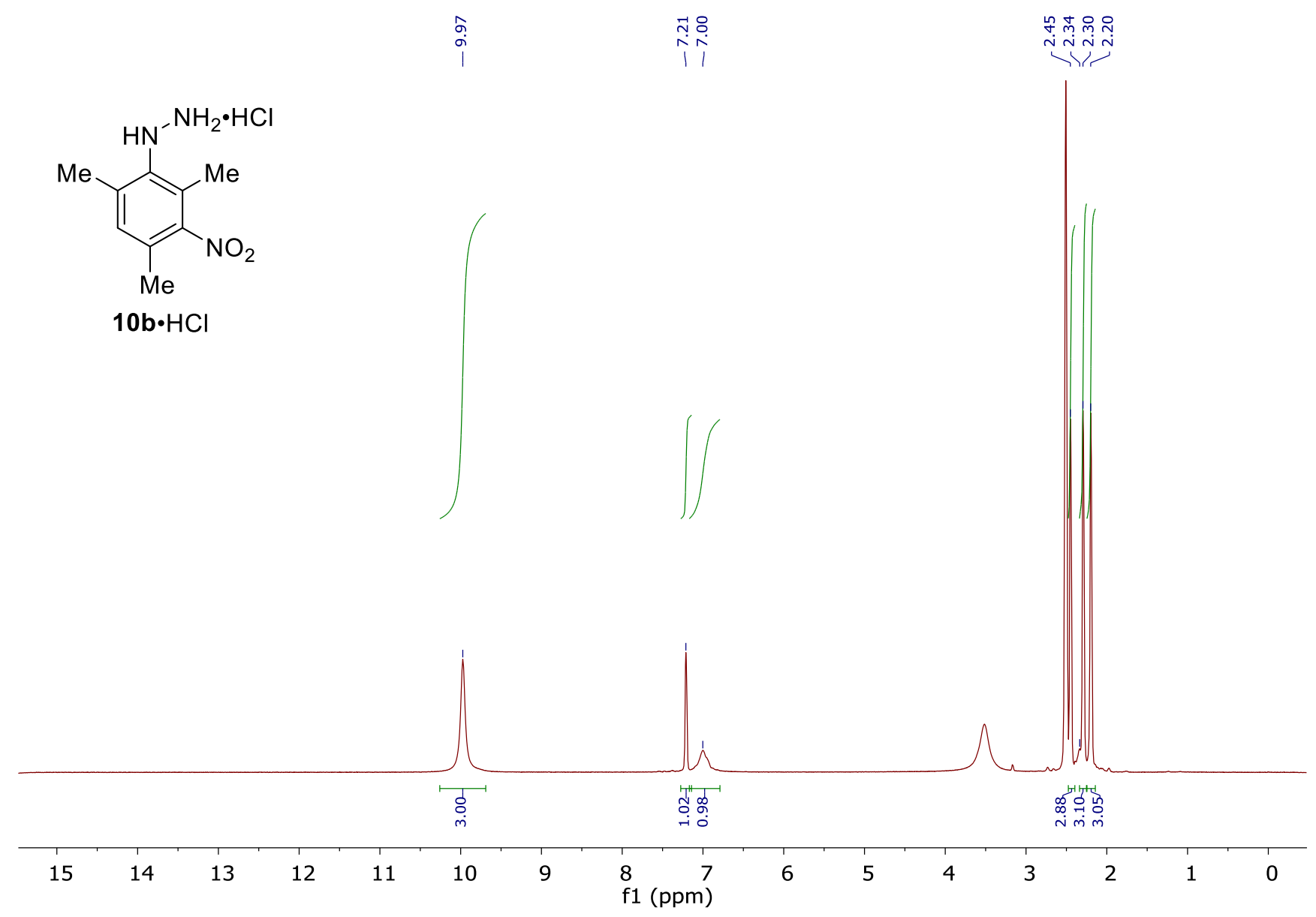

Figure S17. ${ }^{1} \mathrm{H}$ NMR (300 MHz, DMSO- $\left.d_{6}\right)$ of $\mathbf{1 0 b} \cdot \mathrm{HCl}$
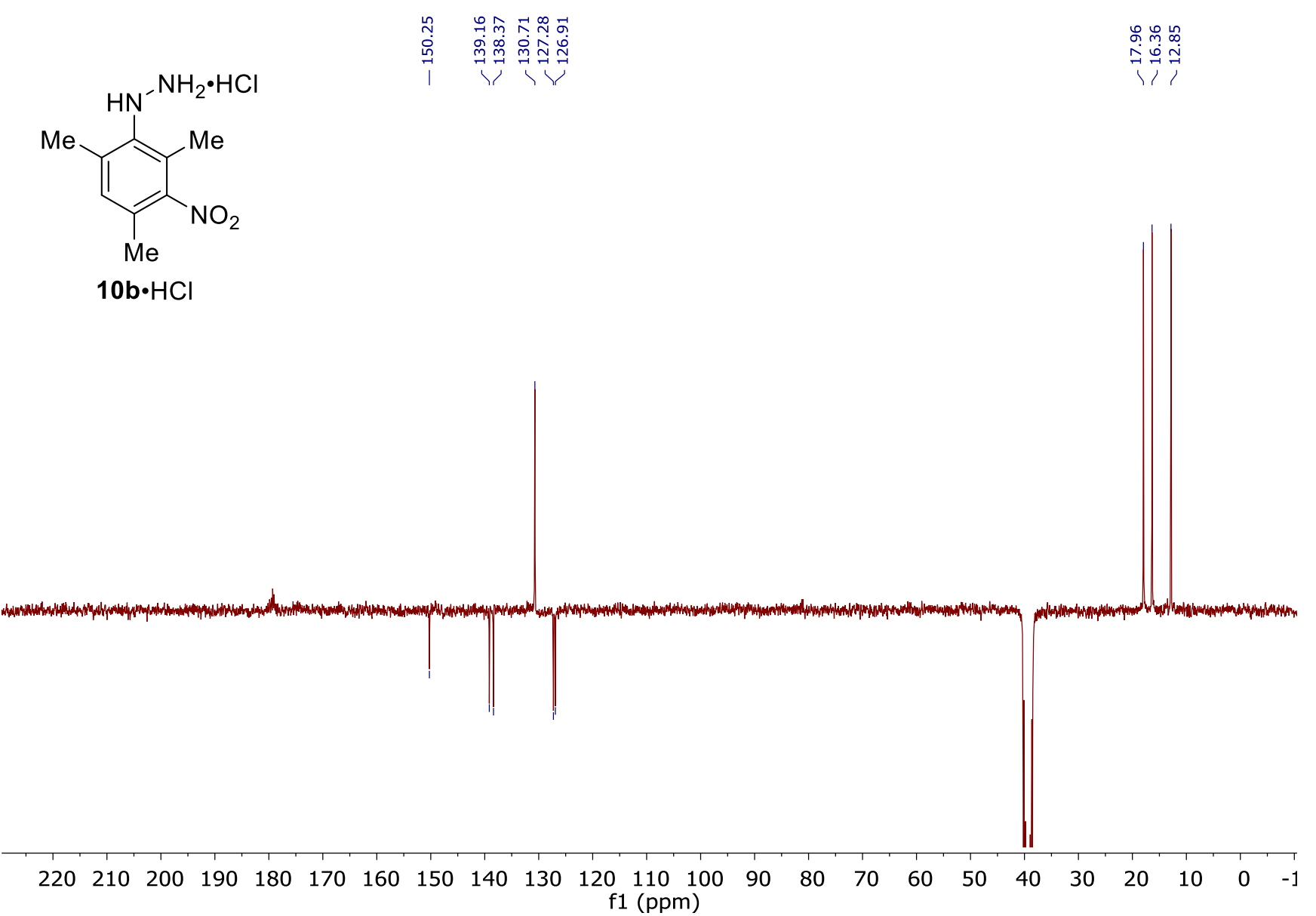

Figure S18. ${ }^{13} \mathrm{C}$ NMR $\left(75 \mathrm{MHz}, \mathrm{APT}, \mathrm{DMSO}-d_{6}\right)$ of $\mathbf{1 0 b} \cdot \mathrm{HCl}$ 


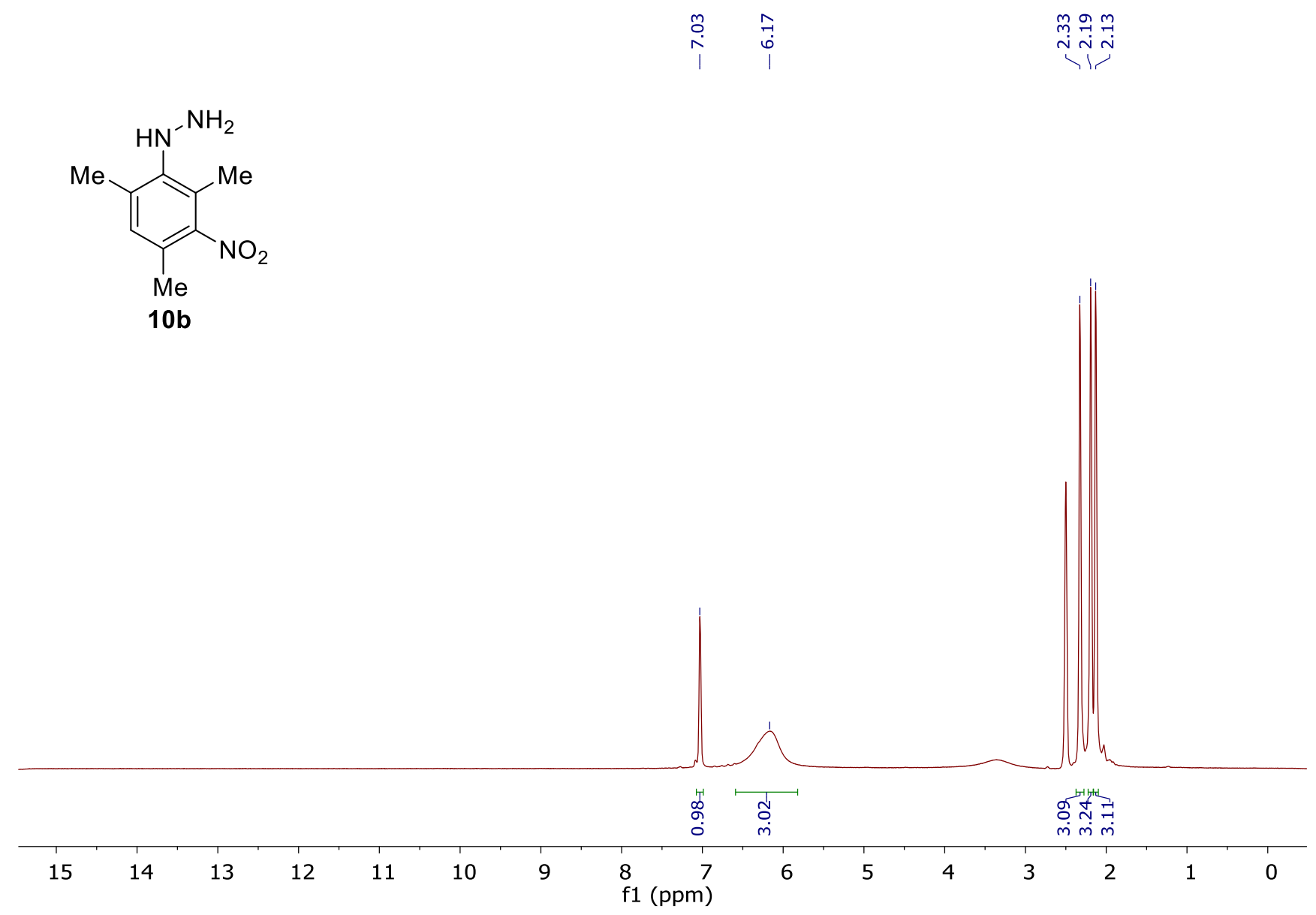

Figure S19. ${ }^{1} \mathrm{H}$ NMR (300 MHz, DMSO-d $)$ of $\mathbf{1 0 b}$

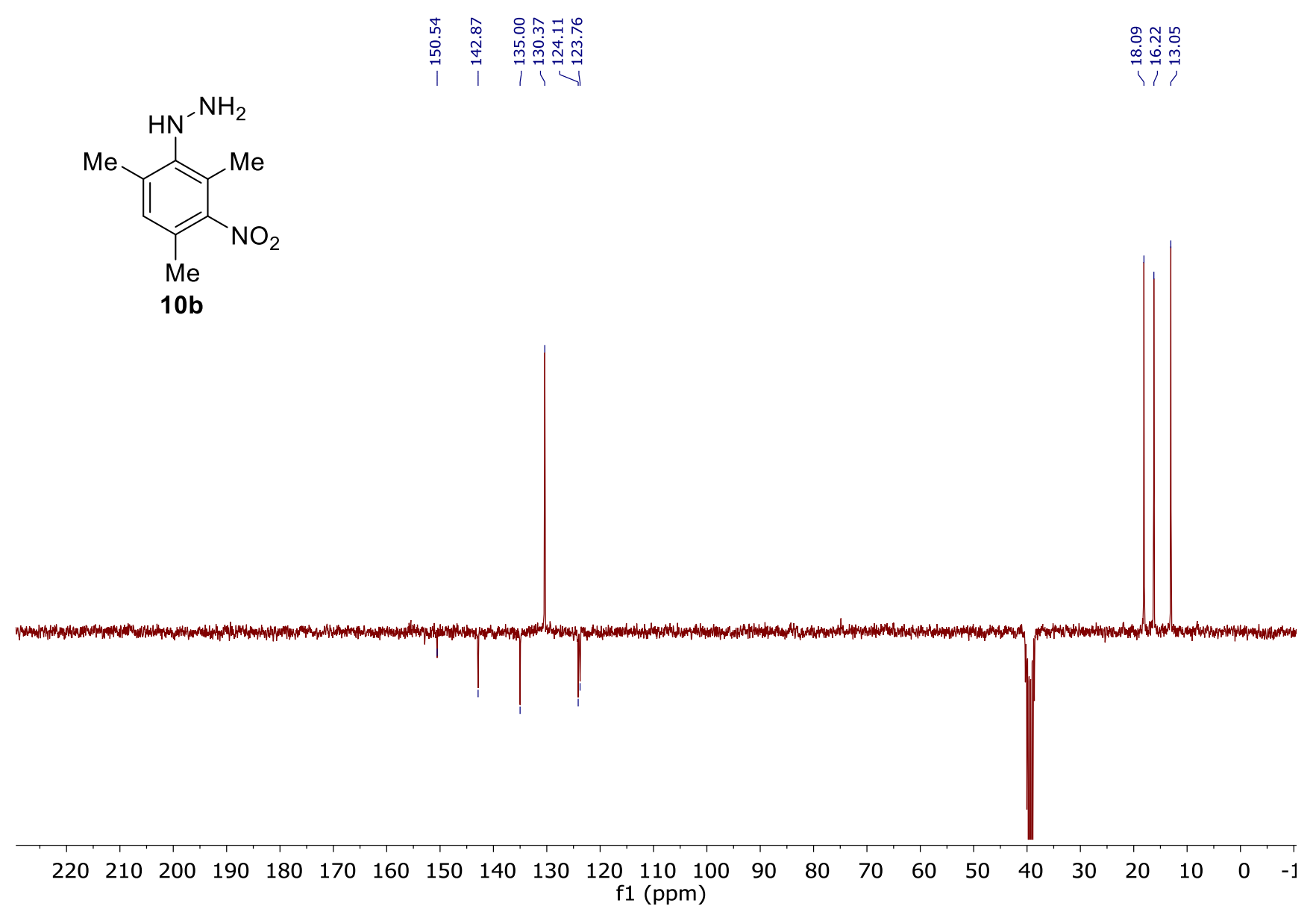

Figure S20. ${ }^{13} \mathrm{C}$ NMR (75 MHz, APT, DMSO- $\left.d_{6}\right)$ of 10b 
<smiles>Cc1ccc([N+](=O)[O-])c(NN)c1NCCl</smiles>

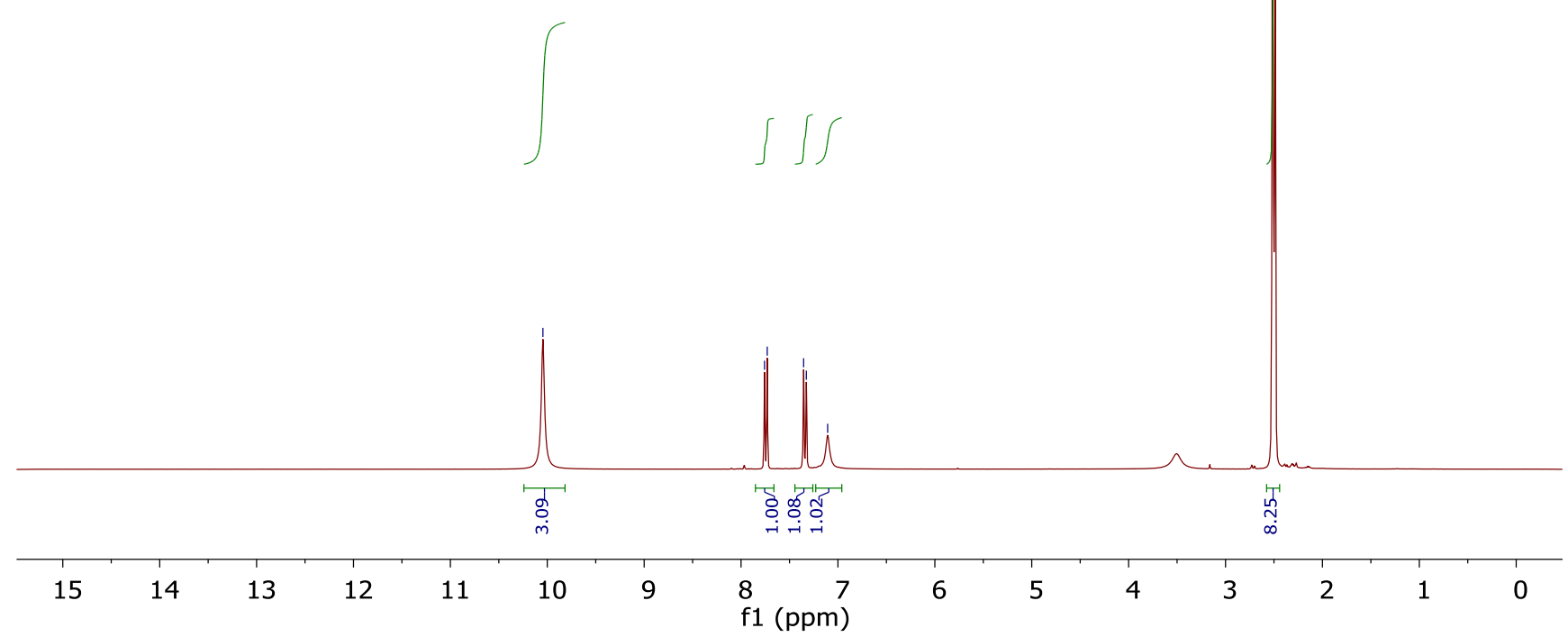

Figure S21. ${ }^{1} \mathrm{H}$ NMR $\left(300 \mathrm{MHz}, \mathrm{DMSO}-d_{6}\right)$ of $\mathbf{1 0 c} \cdot \mathrm{HCl}$<smiles>Cc1ccc([N+](=O)[O-])c(C)c1NNCCl</smiles>

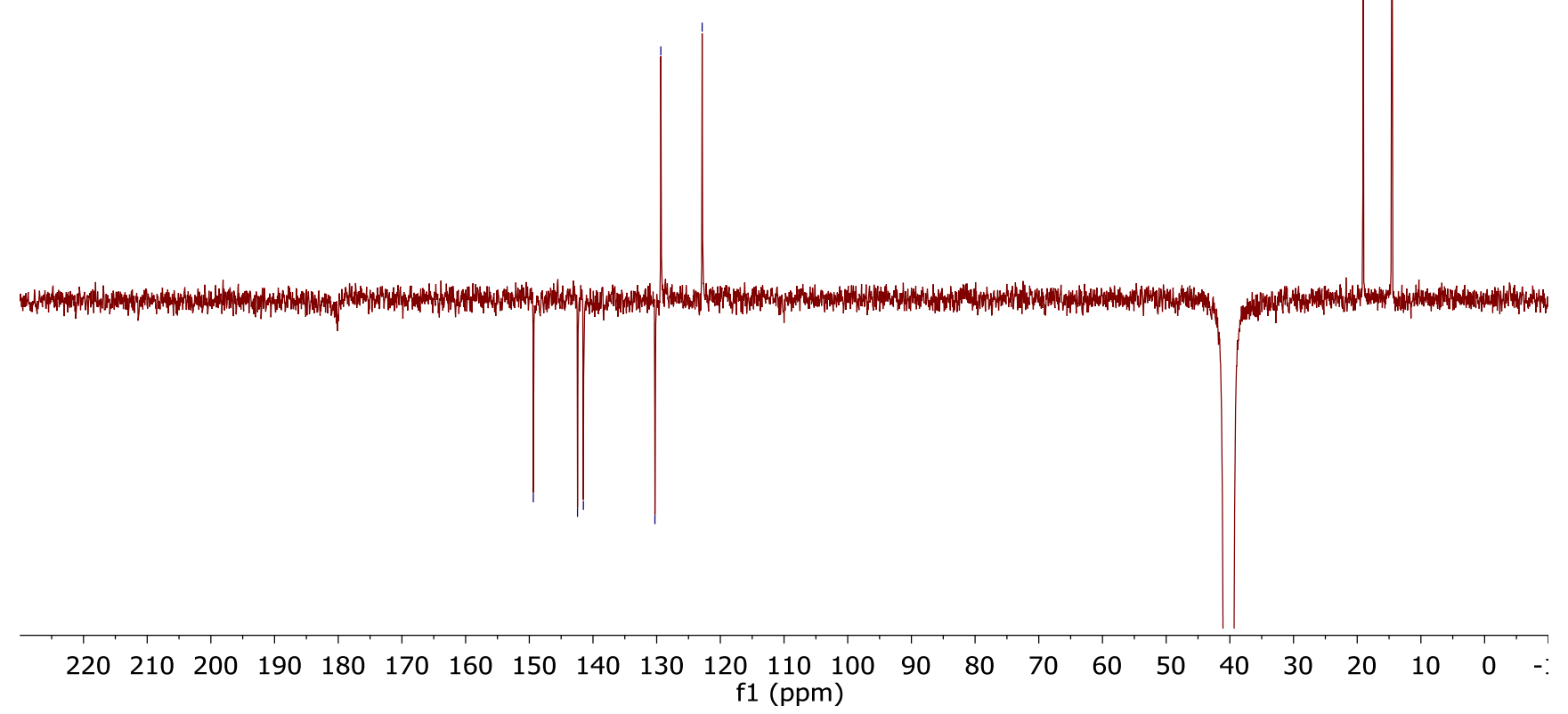

Figure S22. ${ }^{13} \mathrm{C}$ NMR $\left(75 \mathrm{MHz}, \mathrm{APT}, \mathrm{DMSO}-d_{6}\right)$ of $\mathbf{1 0 c} \cdot \mathrm{HCl}$ 
<smiles>Cc1ccc([N+](=O)[O-])c([N+](=O)[O-])c1NN</smiles>

10c

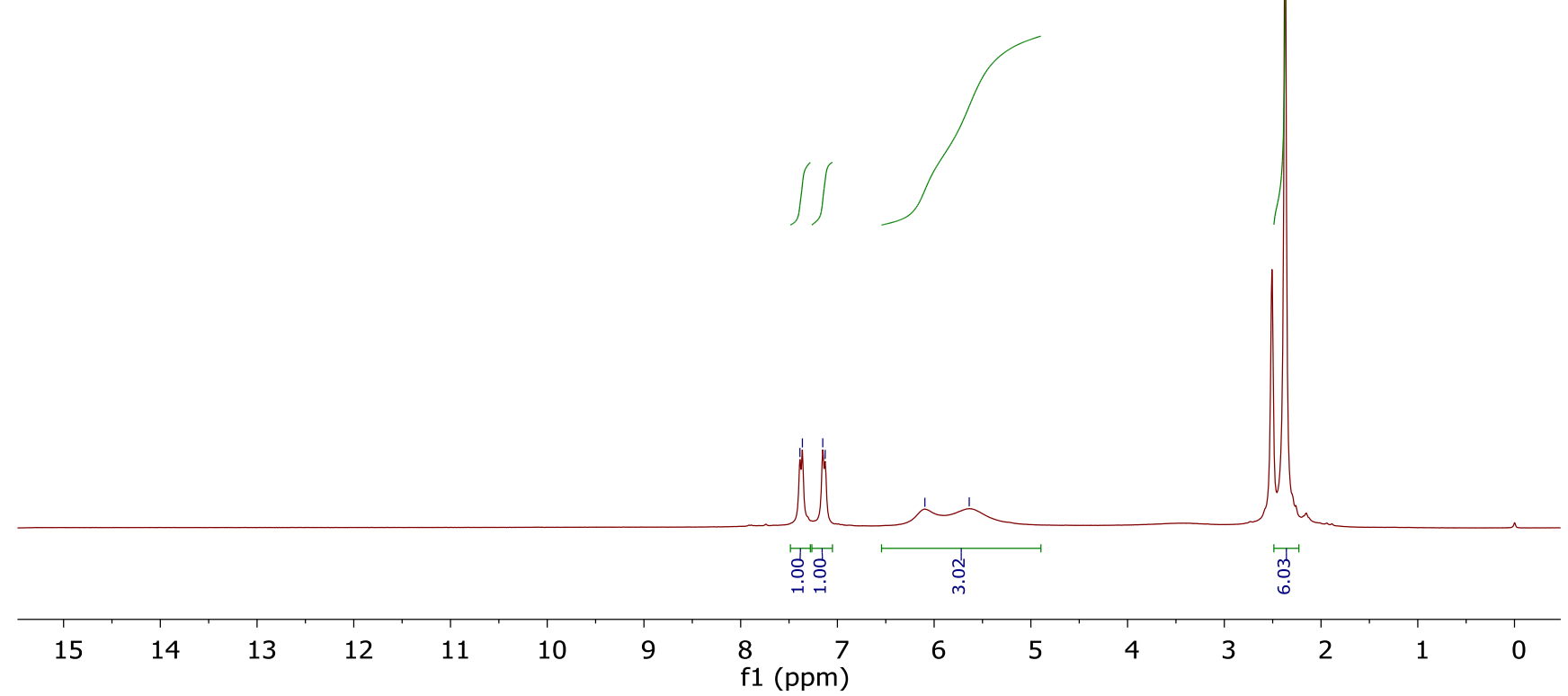

Figure S23. ${ }^{1} \mathrm{H}$ NMR (300 MHz, DMSO- $\left.d_{6}\right)$ of 10c

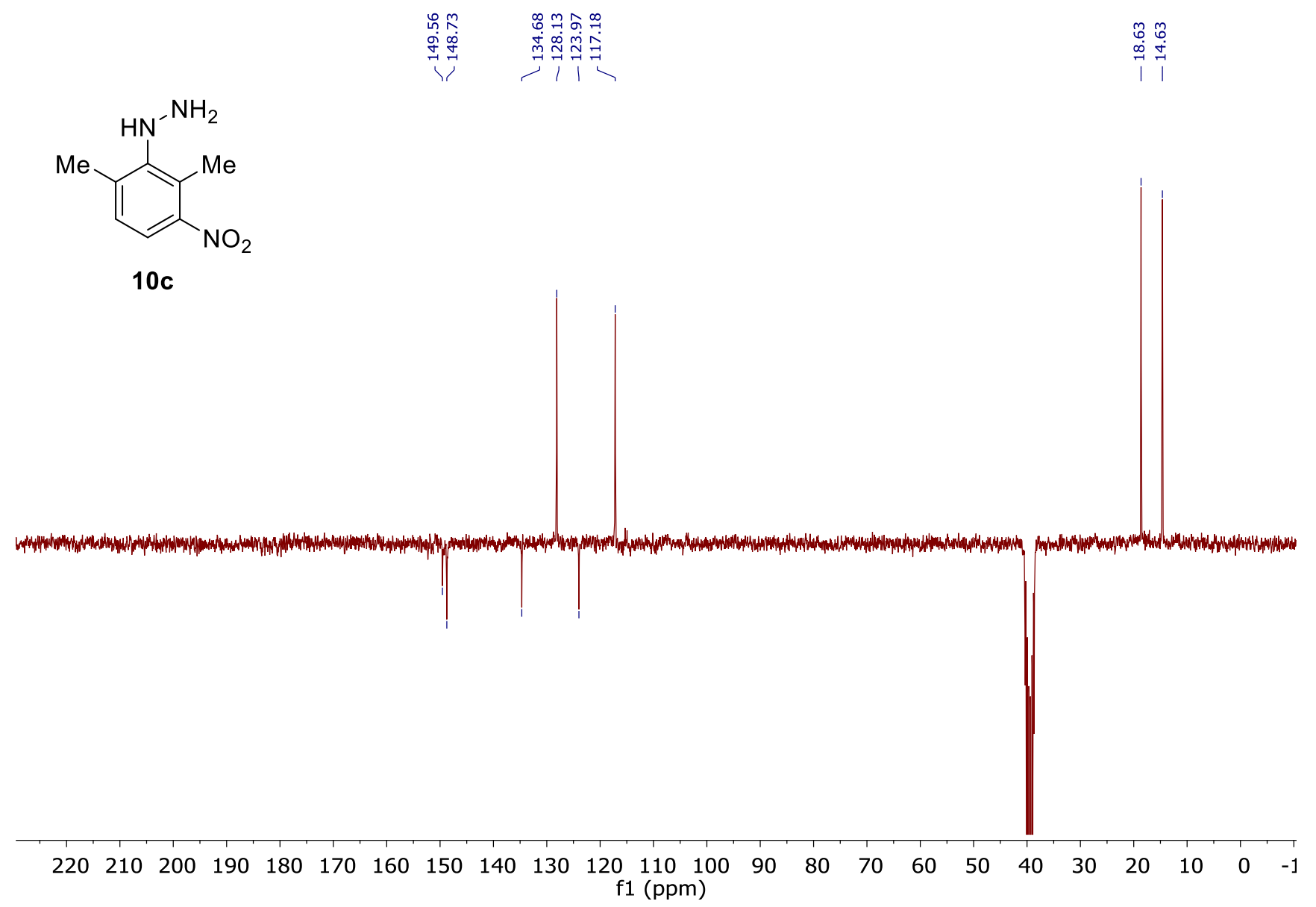

Figure S24. ${ }^{13} \mathrm{C}$ NMR (75 MHz, APT, DMSO- $\left.d_{6}\right)$ of 10c 
<smiles>CCc1ccc([N+](=O)[O-])c(CC)c1NN</smiles>

$10 \mathrm{~d} \bullet \mathrm{HCl}$

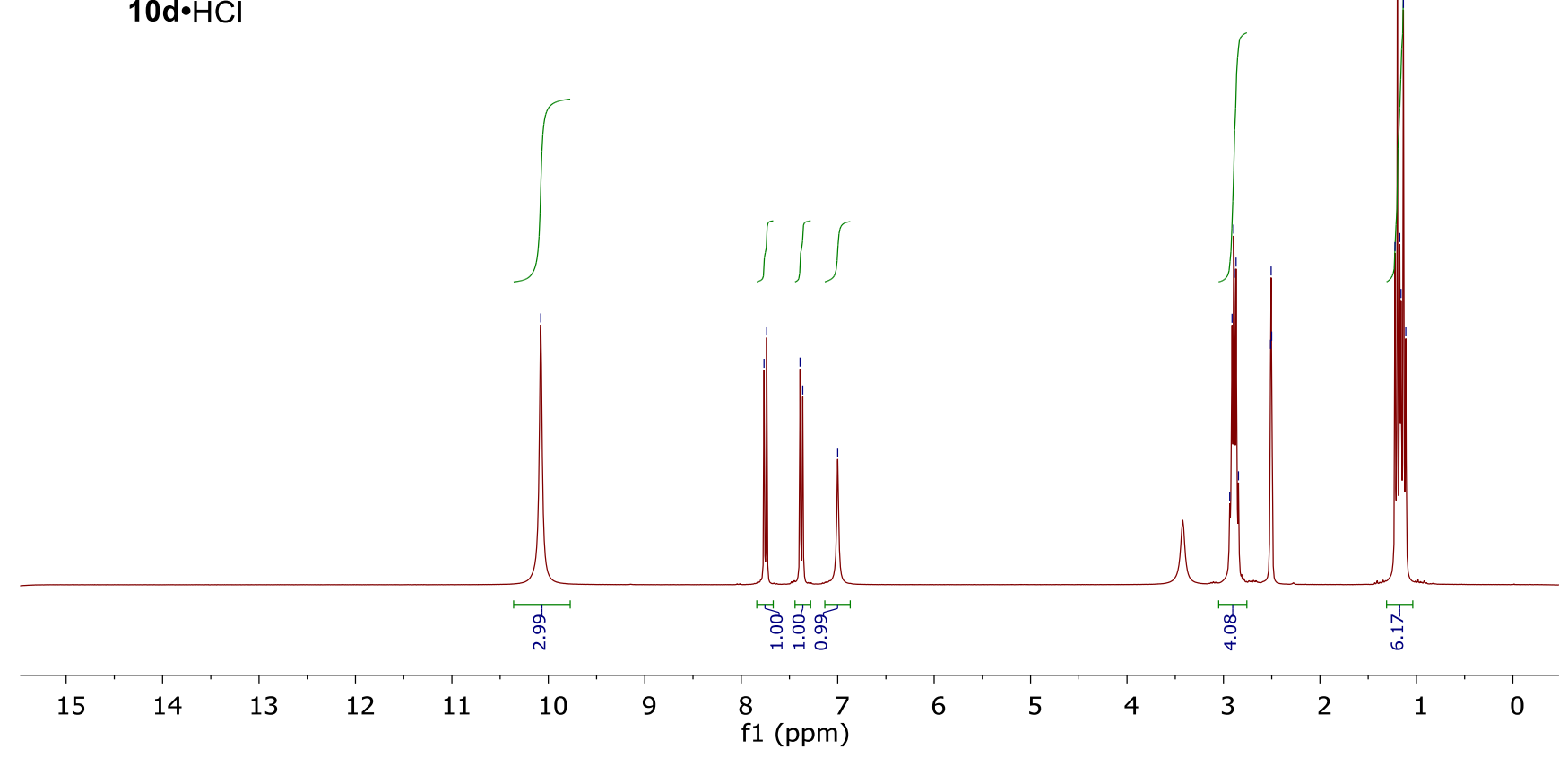

Figure S25. ${ }^{1} \mathrm{H}$ NMR $\left(300 \mathrm{MHz}, \mathrm{DMSO}-d_{6}\right)$ of $\mathbf{1 0 d} \cdot \mathrm{HCl}$

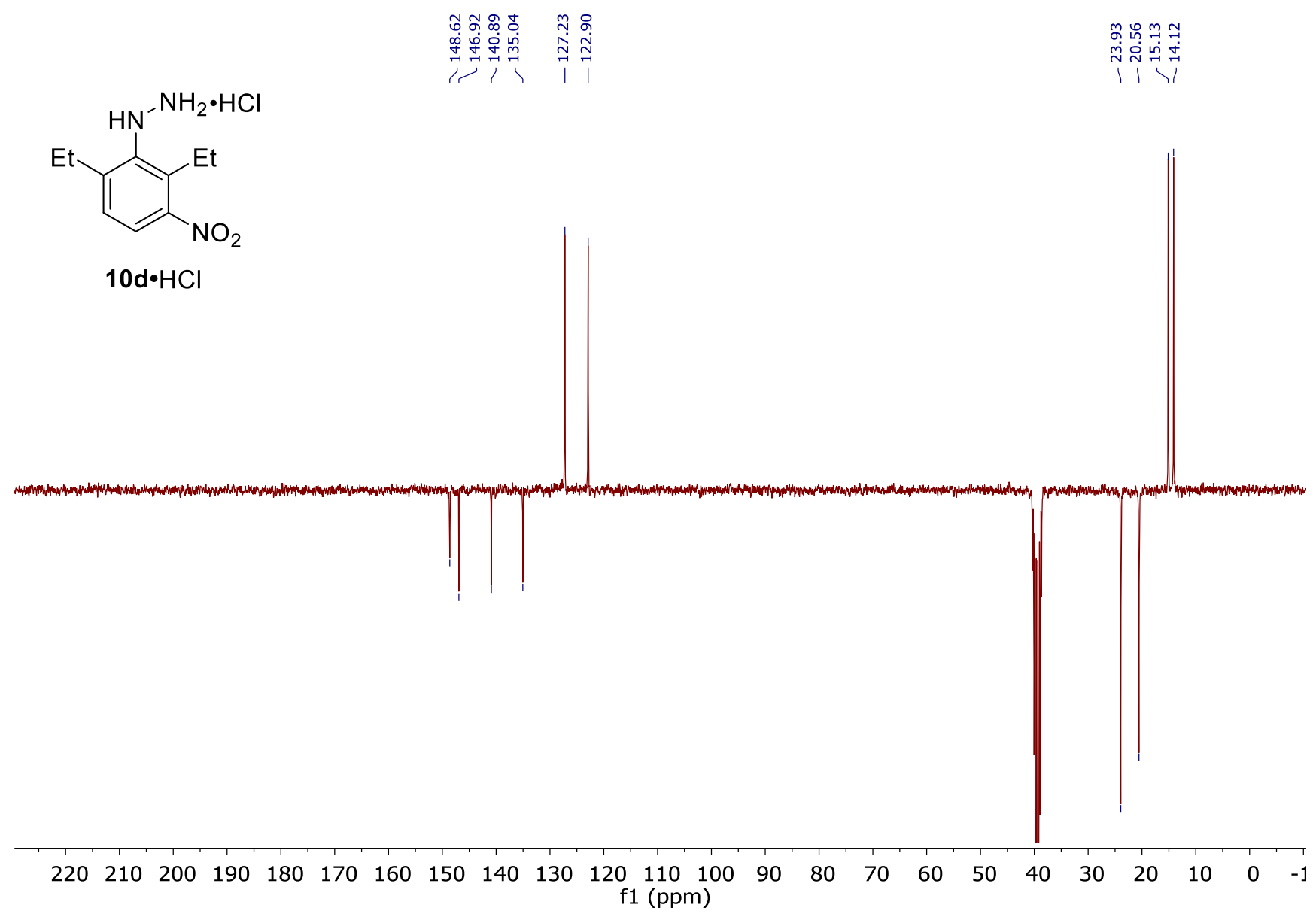

Figure S26. ${ }^{13} \mathrm{C}$ NMR $\left(75 \mathrm{MHz}, \mathrm{APT}, \mathrm{DMSO}-d_{6}\right)$ of $\mathbf{1 0 d} \cdot \mathrm{HCl}$ 


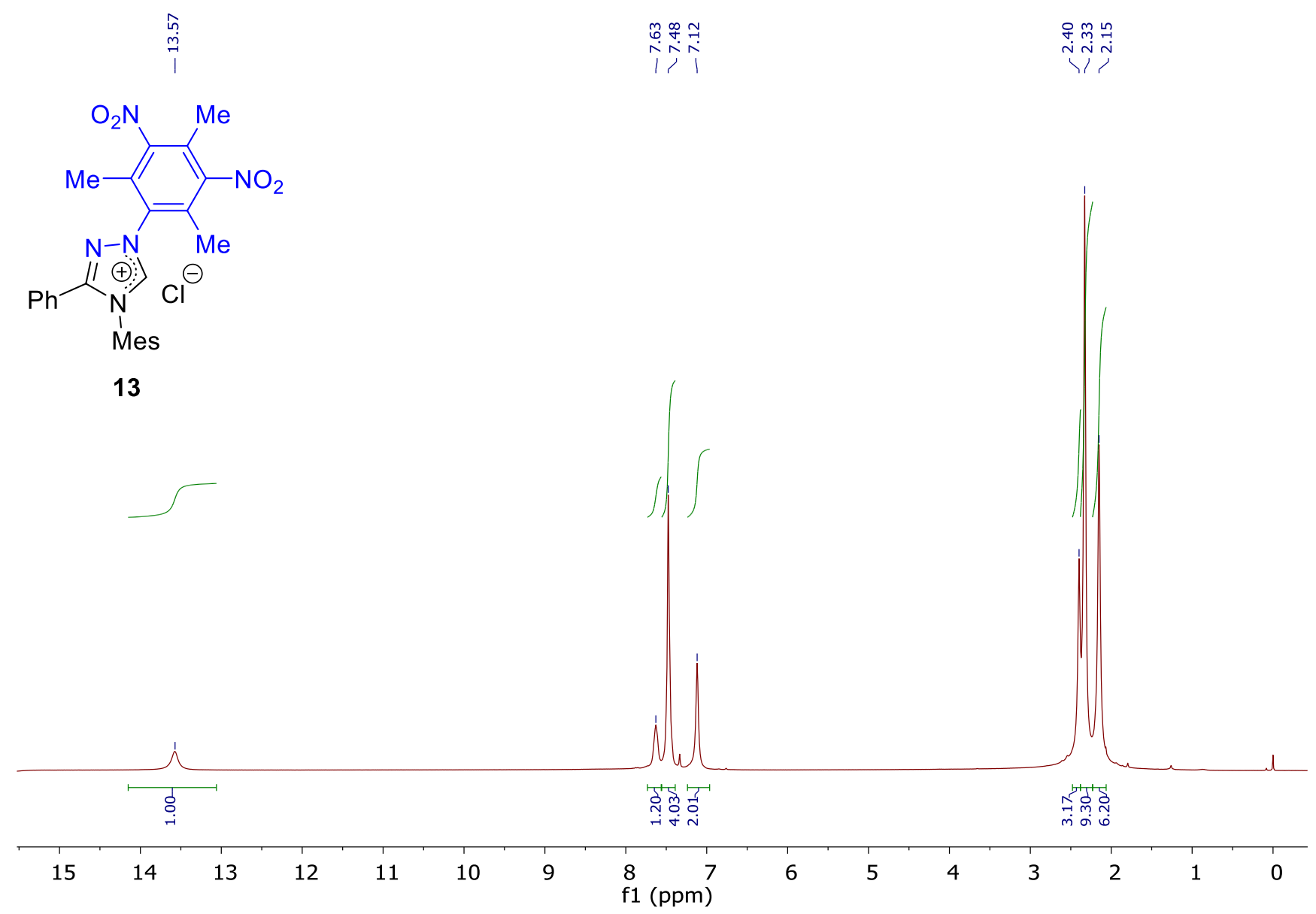

Figure S27. ${ }^{1} \mathrm{H}$ NMR $\left(300 \mathrm{MHz}, \mathrm{CDCl}_{3}\right)$ of $\mathbf{1 3}$

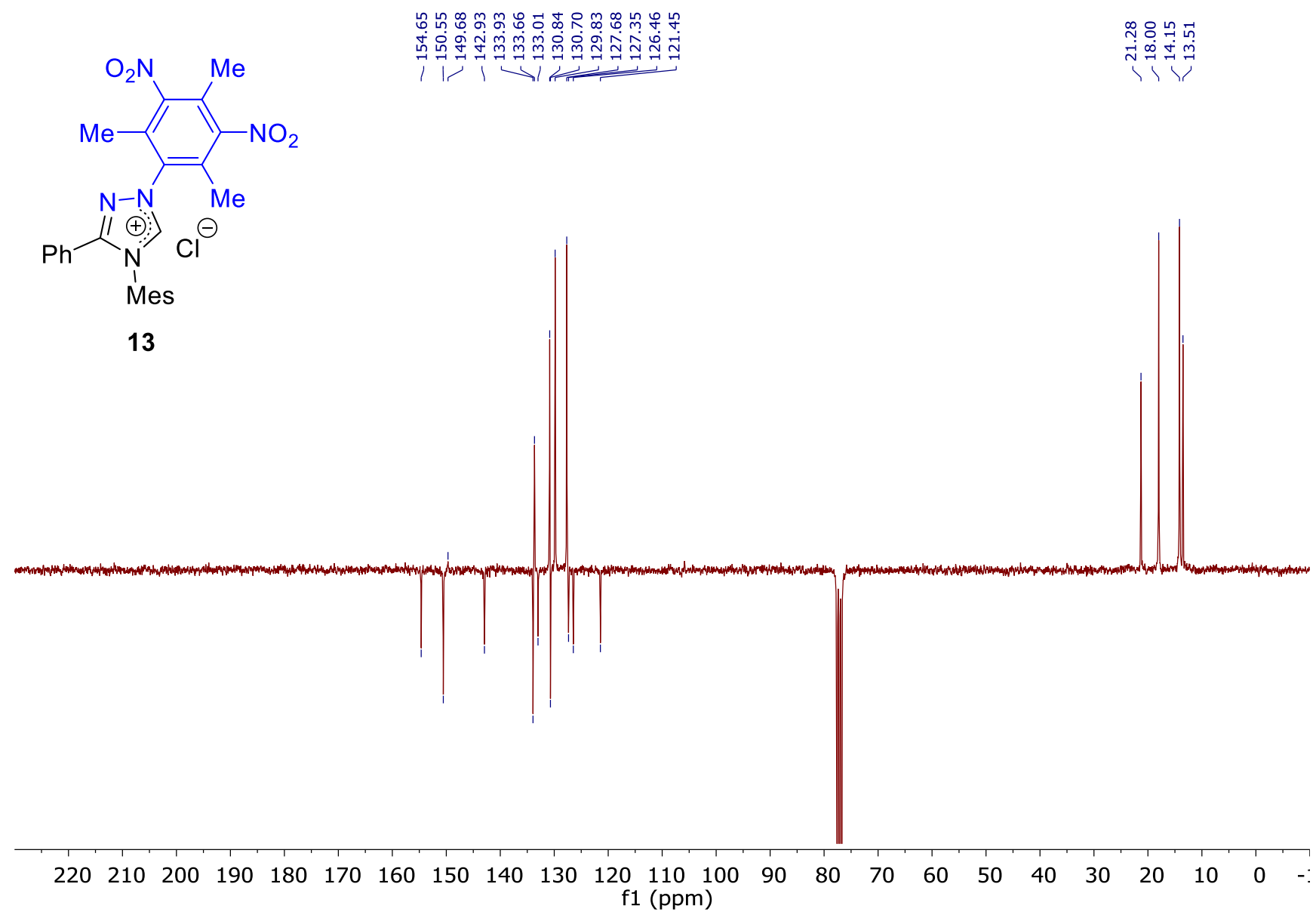

Figure S28. ${ }^{13} \mathrm{C}$ NMR $\left(75 \mathrm{MHz}, \mathrm{APT}, \mathrm{CDCl}_{3}\right)$ of $\mathbf{1 3}$ 


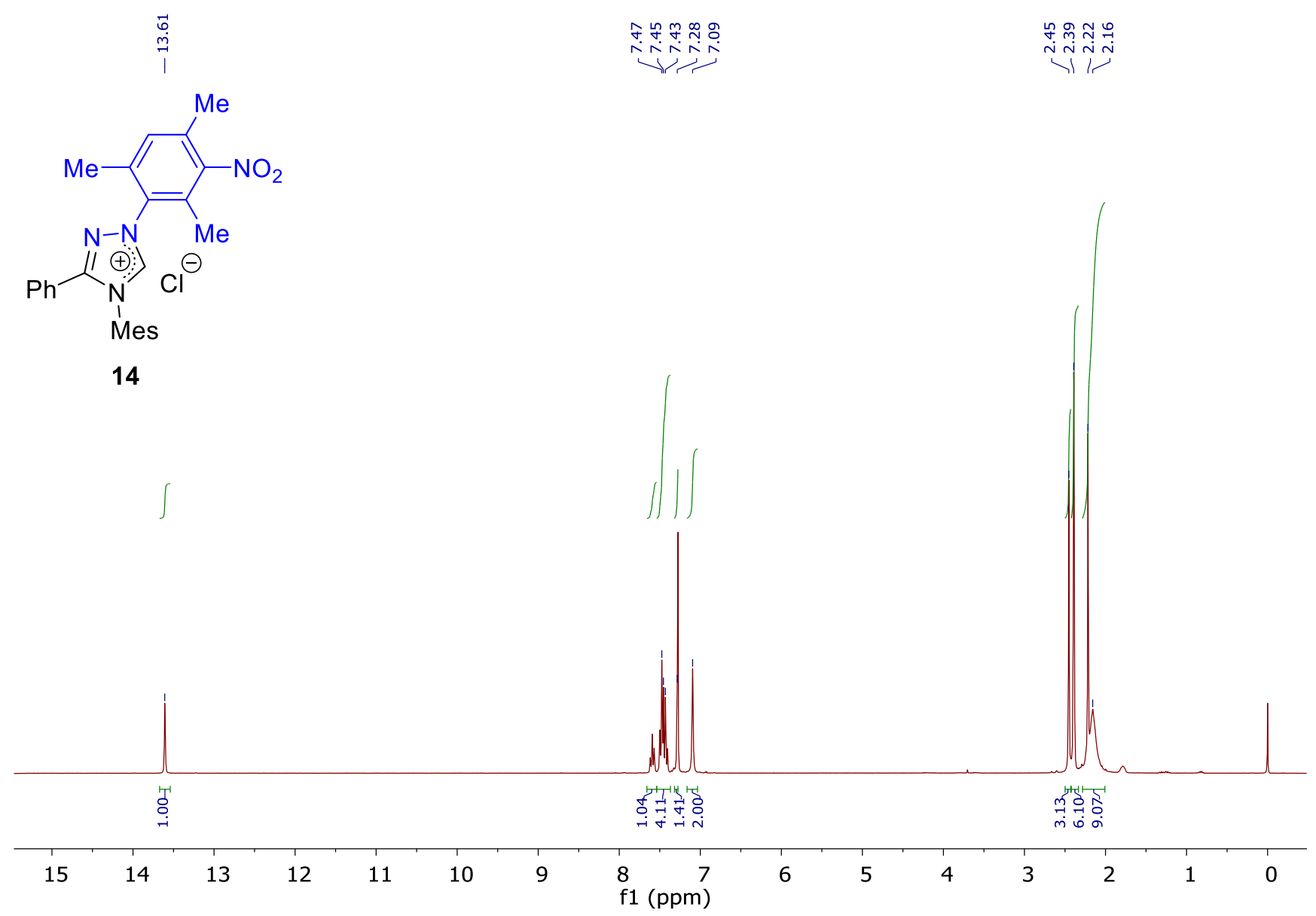

Figure S29. ${ }^{1} \mathrm{H}$ NMR $\left(300 \mathrm{MHz}, \mathrm{CDCl}_{3}\right)$ of $\mathbf{1 4}$

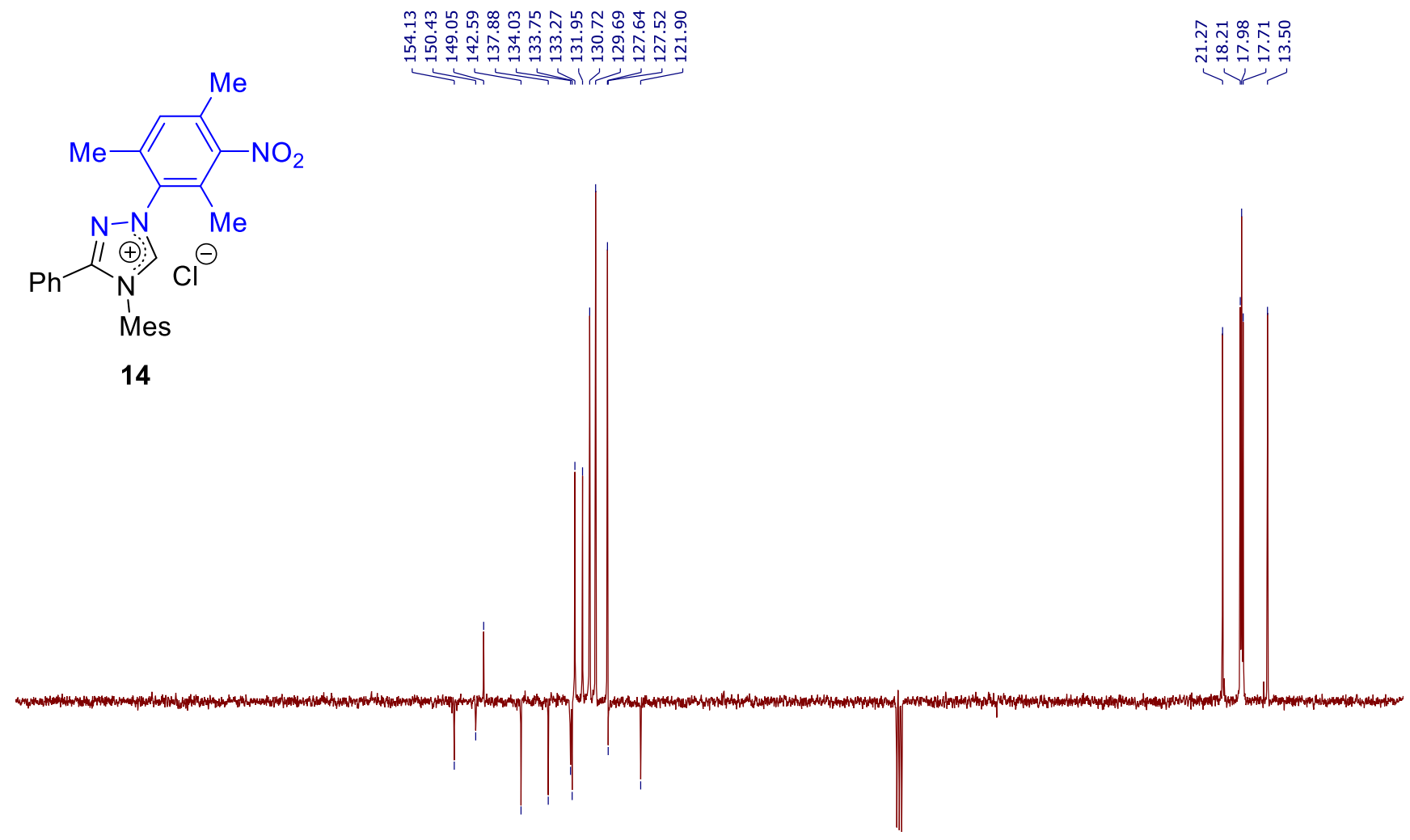

$\begin{array}{lllllllllllllllllllllll}220 & 210 & 200 & 190 & 180 & 170 & 160 & 150 & 140 & 130 & \begin{array}{c}120 \\ \mathrm{f} 1\end{array} \begin{array}{l}110 \\ (\mathrm{ppm})\end{array} & 100 & 90 & 80 & 70 & 60 & 50 & 40 & 30 & 20 & 10 & 0 & -\end{array}$

Figure S30. ${ }^{13} \mathrm{C} \mathrm{NMR}\left(75 \mathrm{MHz}, \mathrm{APT}, \mathrm{CDCl}_{3}\right)$ of $\mathbf{1 4}$ 


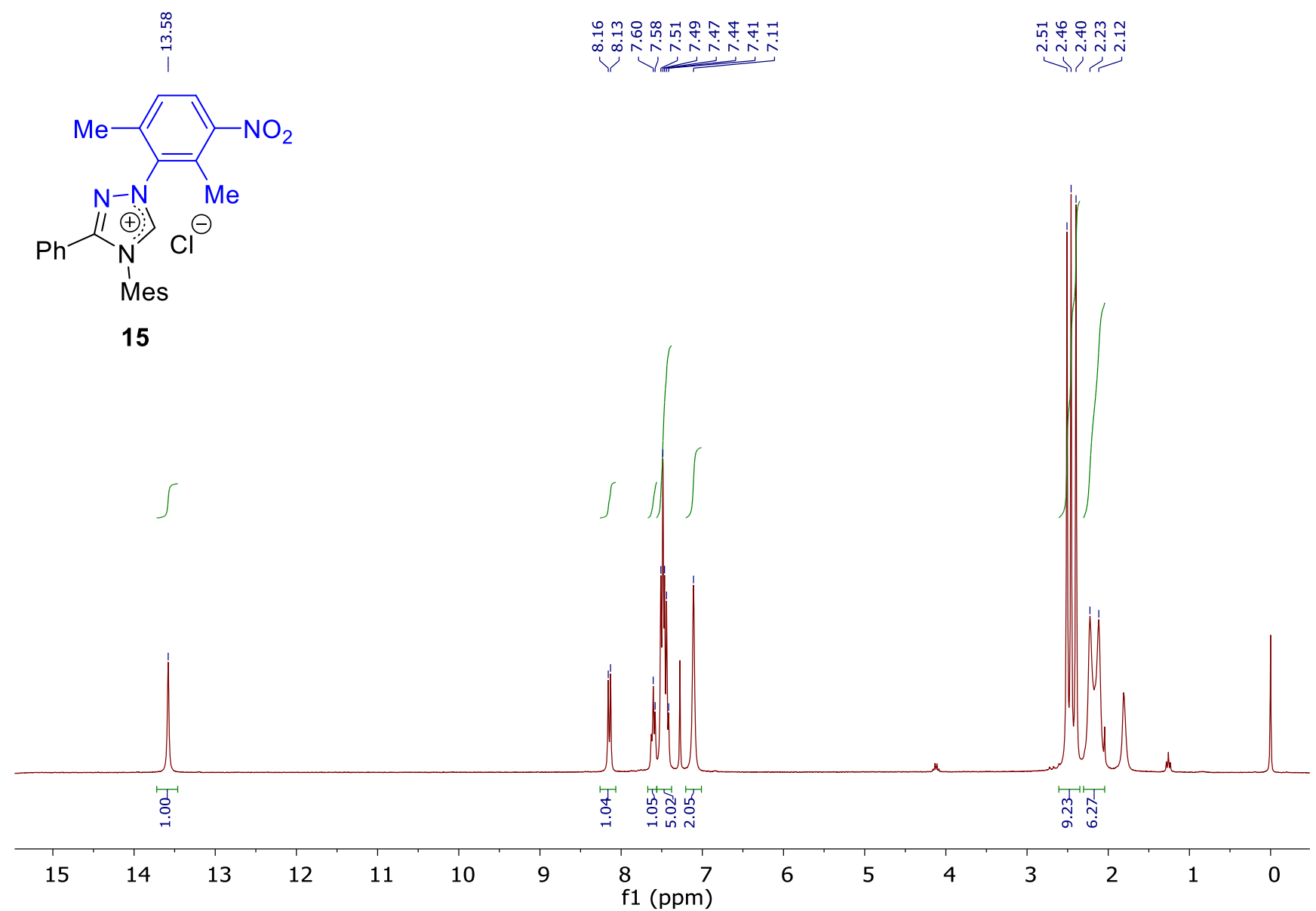

Figure S31. ${ }^{1} \mathrm{H} \mathrm{NMR}\left(300 \mathrm{MHz}, \mathrm{CDCl}_{3}\right)$ of $\mathbf{1 5}$

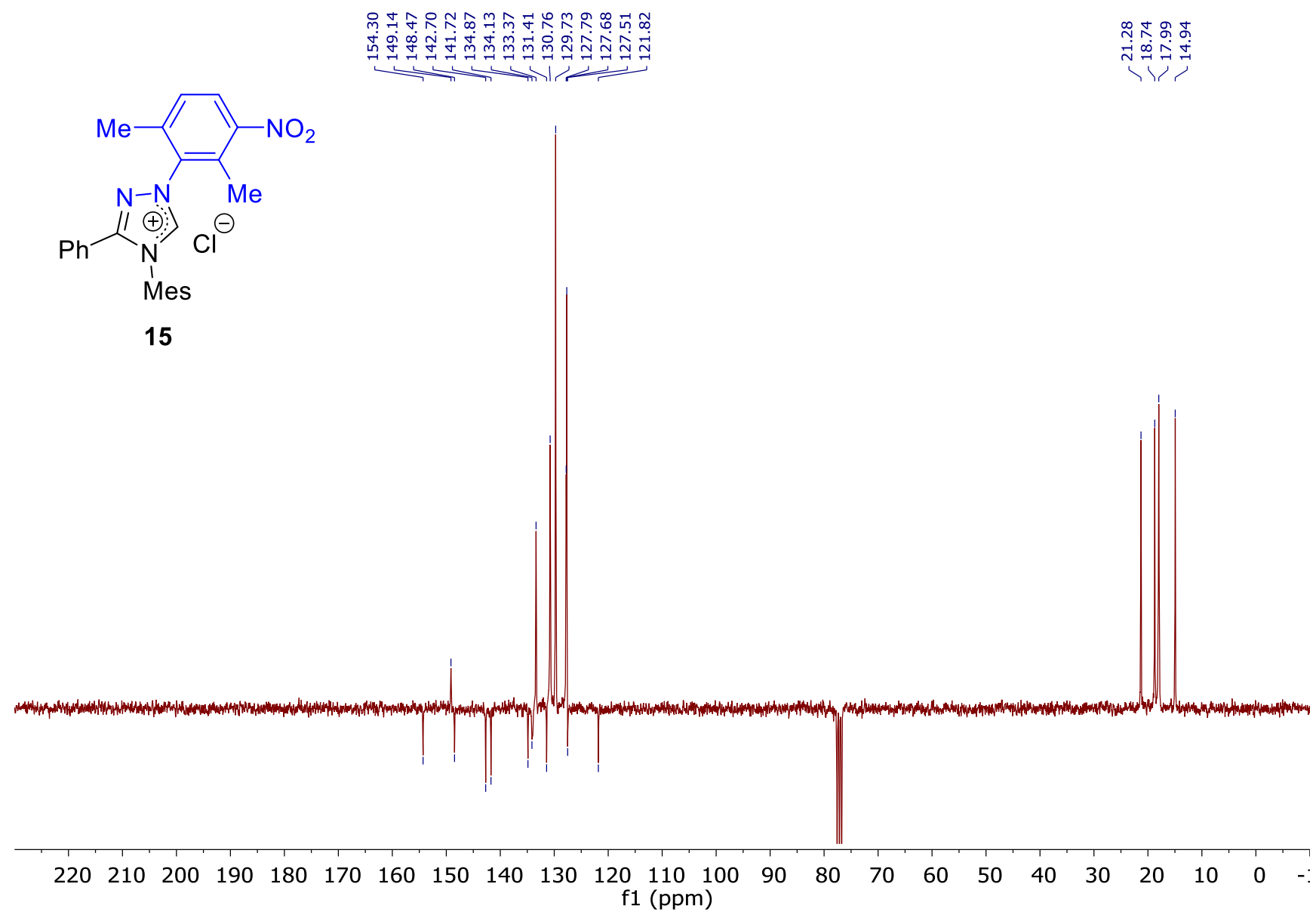

Figure S32. ${ }^{13} \mathrm{C}$ NMR ( $\left.75 \mathrm{MHz}, \mathrm{APT}, \mathrm{CDCl}_{3}\right)$ of $\mathbf{1 5}$ 


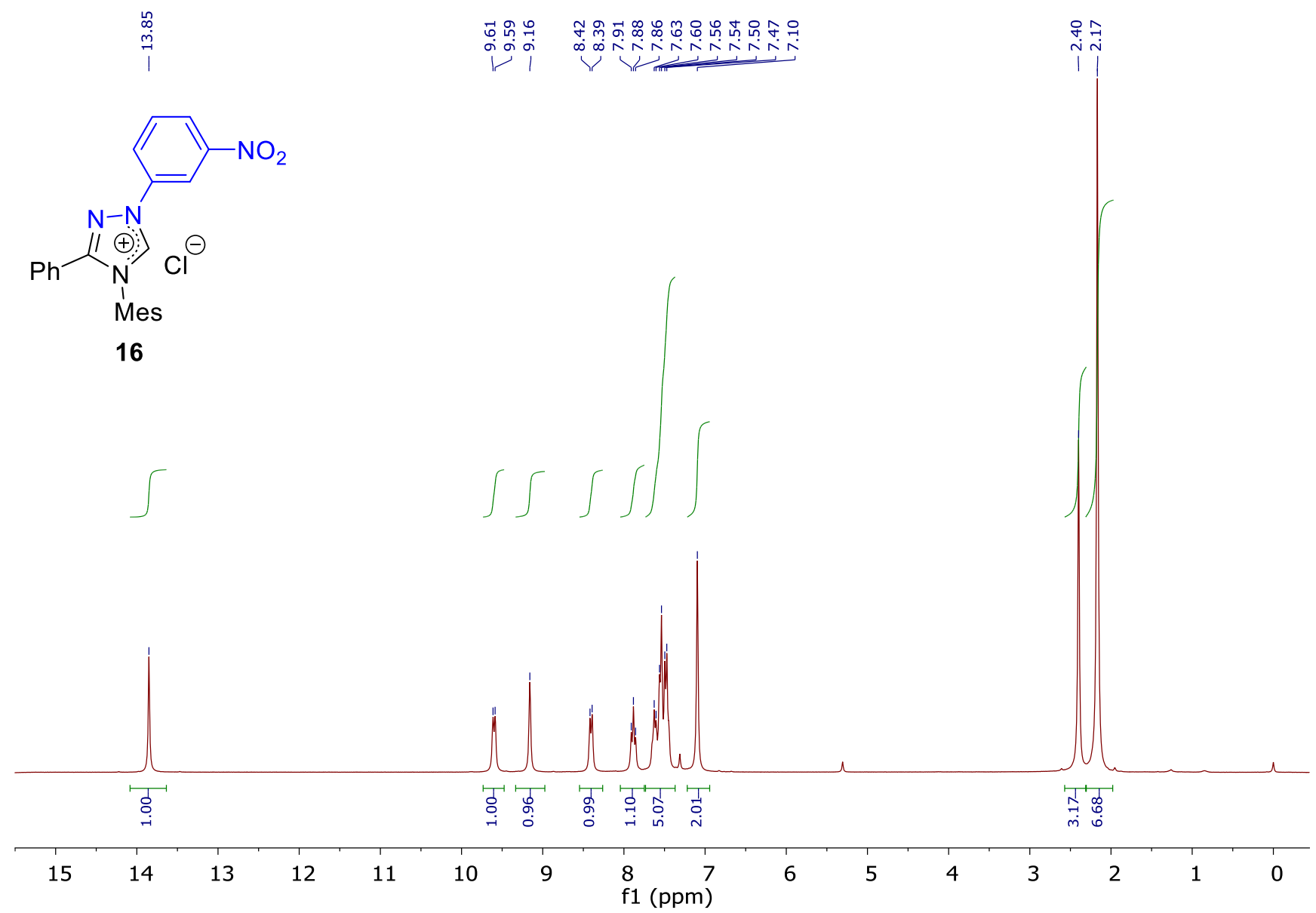

Figure S33. ${ }^{1} \mathrm{H}$ NMR $\left(300 \mathrm{MHz}, \mathrm{CDCl}_{3}\right)$ of $\mathbf{1 6}$

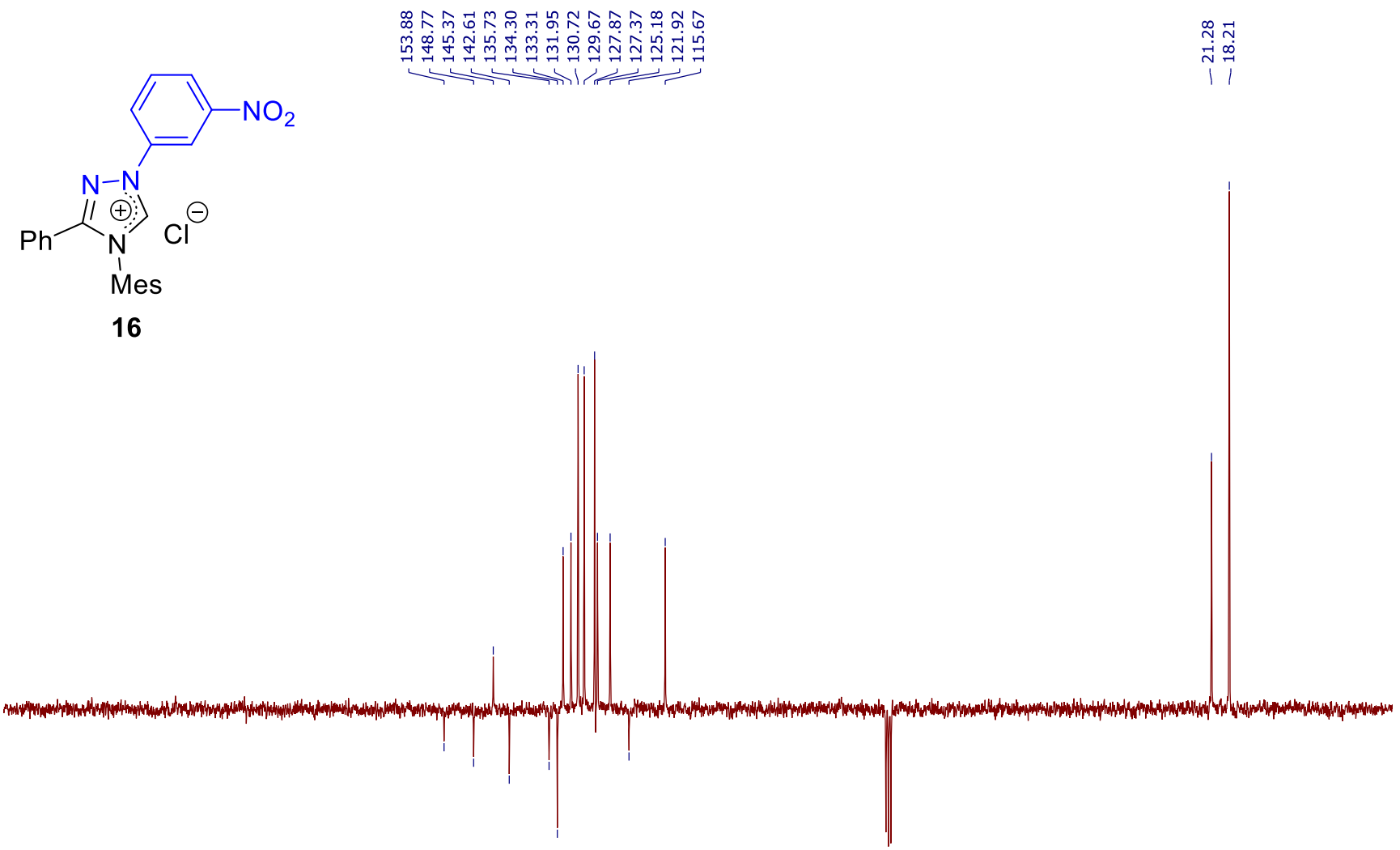

$\begin{array}{lllllllllllllllllllllllll}220 & 210 & 200 & 190 & 180 & 170 & 160 & 150 & 140 & 130 & 120 & 110 & 100 & 90 & 80 & 70 & 60 & 50 & 40 & 30 & 20 & 10 & 0 & -:\end{array}$

Figure S34. ${ }^{13} \mathrm{C}$ NMR $\left(75 \mathrm{MHz}, \mathrm{APT}, \mathrm{CDCl}_{3}\right)$ of $\mathbf{1 6}$ 


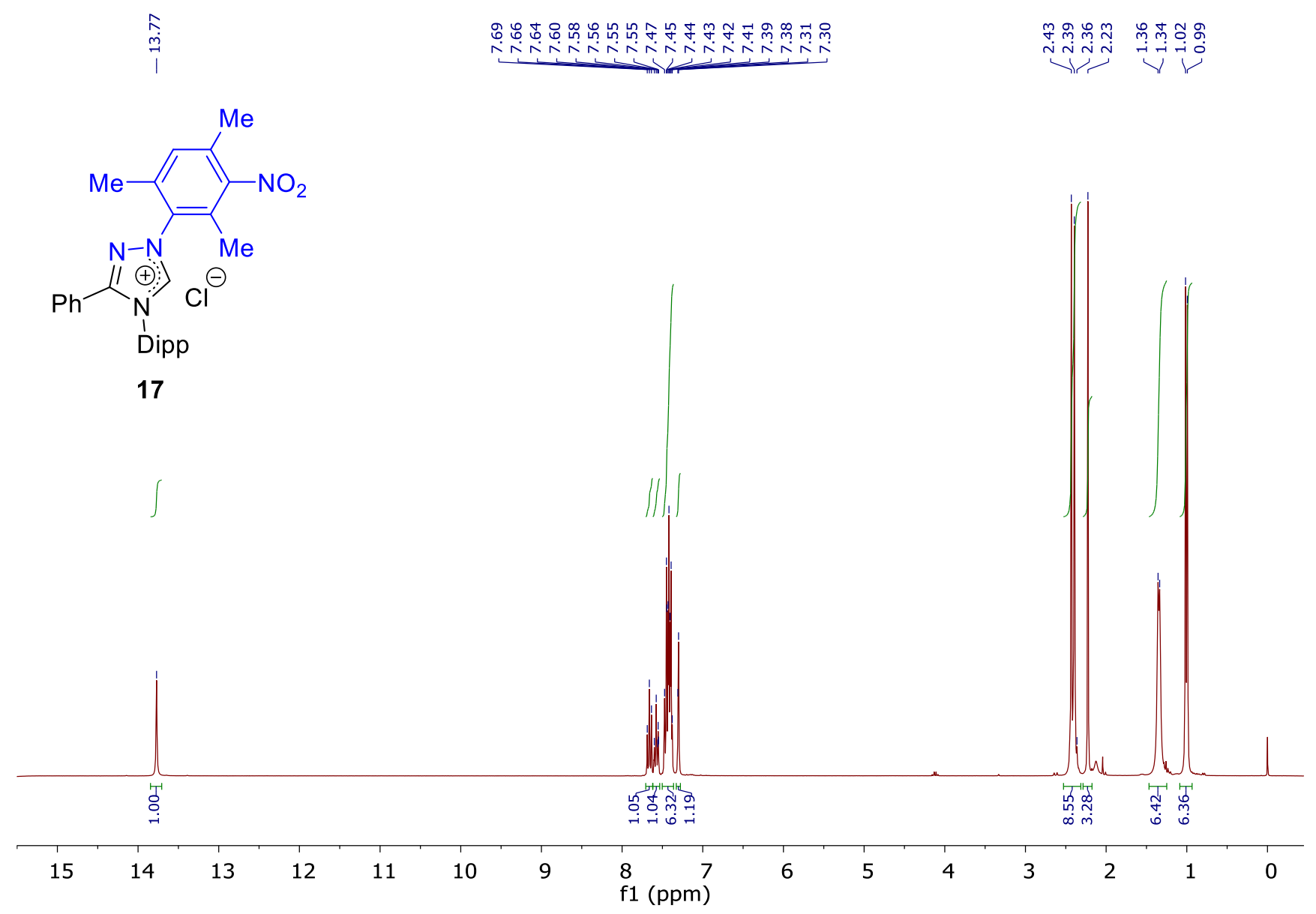

Figure S35. ${ }^{1} \mathrm{H} \mathrm{NMR}\left(300 \mathrm{MHz}, \mathrm{CDCl}_{3}\right)$ of $\mathbf{1 7}$

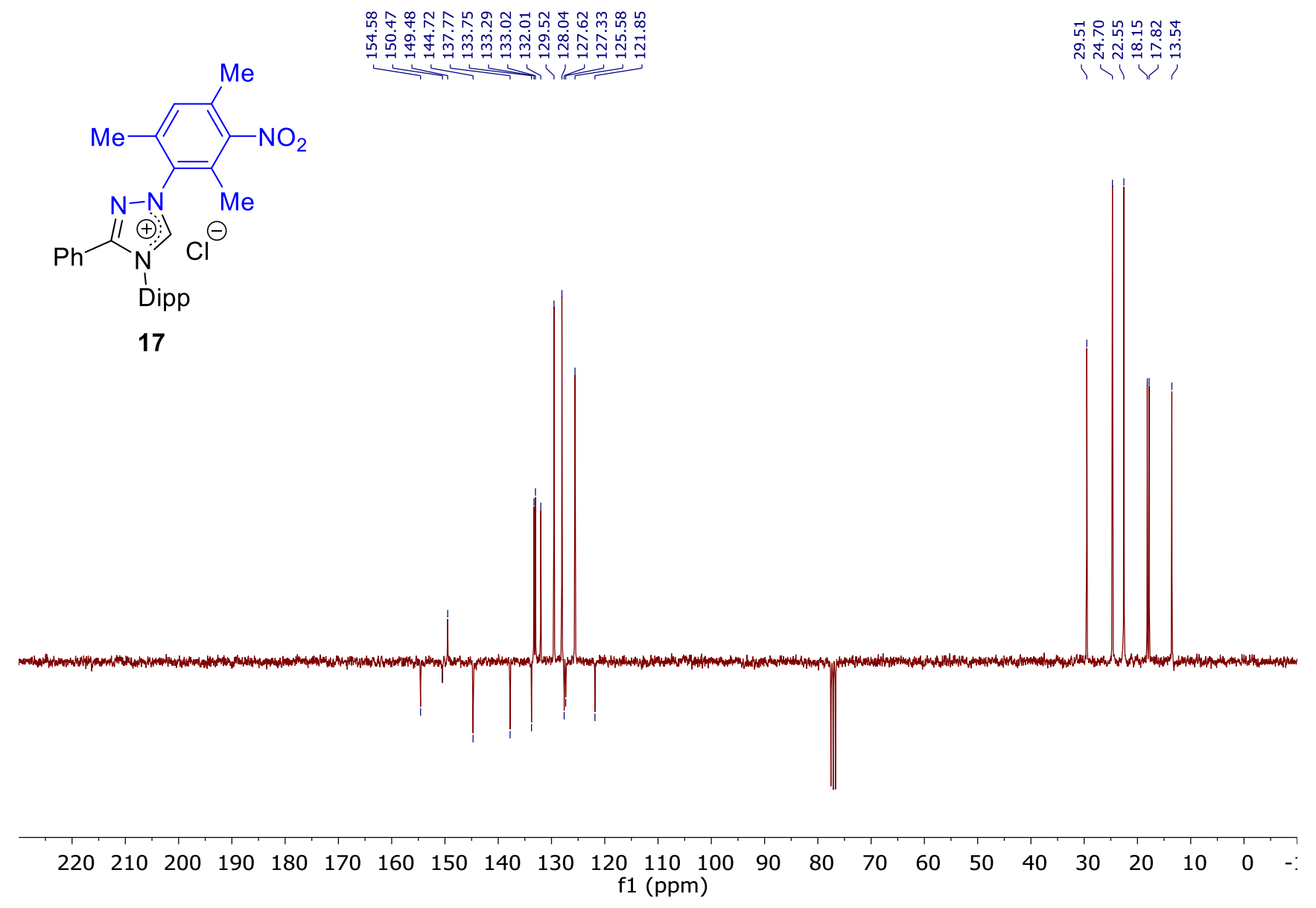

Figure S36. ${ }^{13} \mathrm{C}$ NMR $\left(75 \mathrm{MHz}, \mathrm{APT}, \mathrm{CDCl}_{3}\right)$ of $\mathbf{1 7}$ 


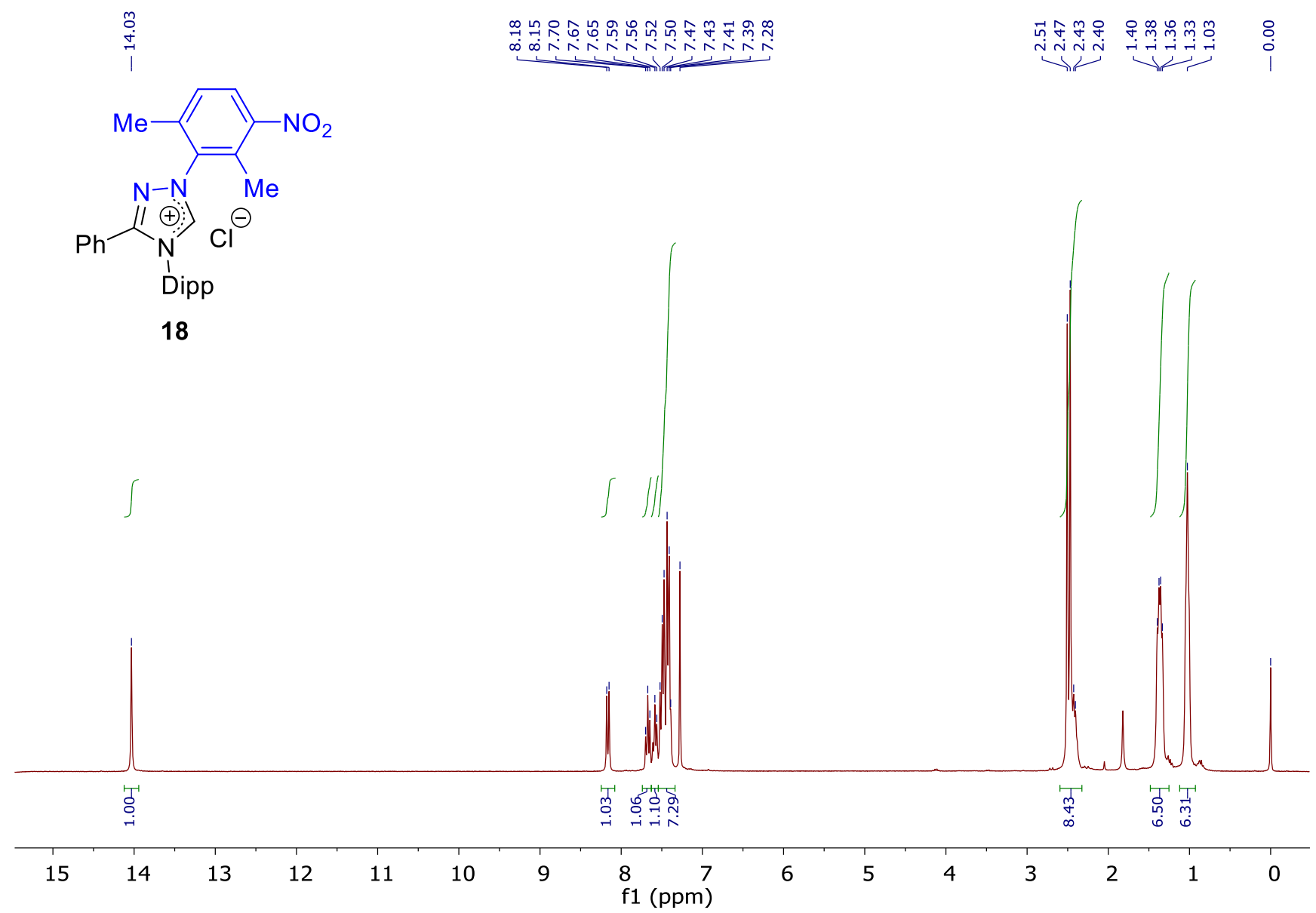

Figure S37. ${ }^{1} \mathrm{H} \mathrm{NMR}\left(300 \mathrm{MHz}, \mathrm{CDCl}_{3}\right)$ of $\mathbf{1 8}$

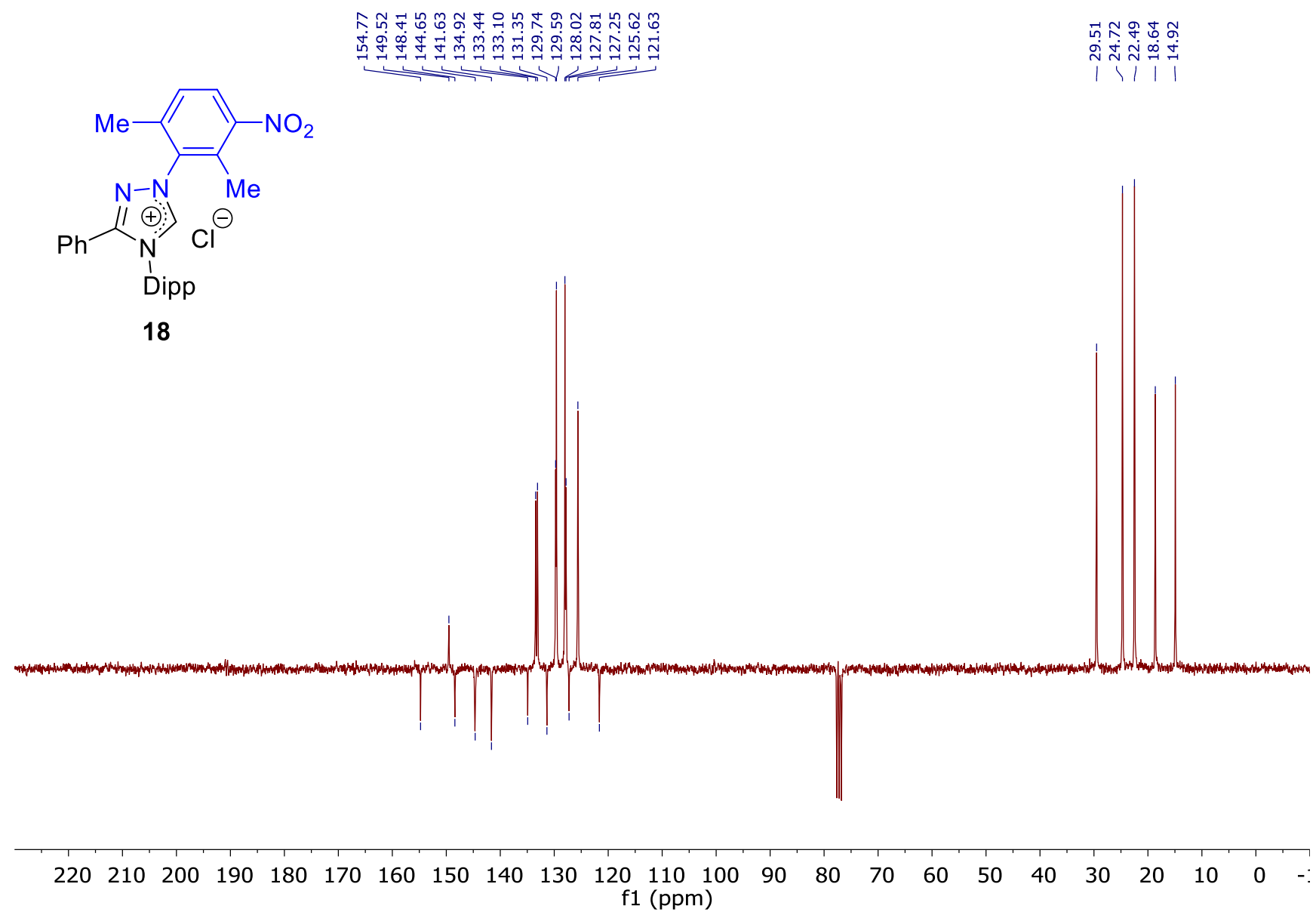

Figure S38. ${ }^{13} \mathrm{C}$ NMR $\left(75 \mathrm{MHz}, \mathrm{APT}, \mathrm{CDCl}_{3}\right)$ of $\mathbf{1 8}$ 


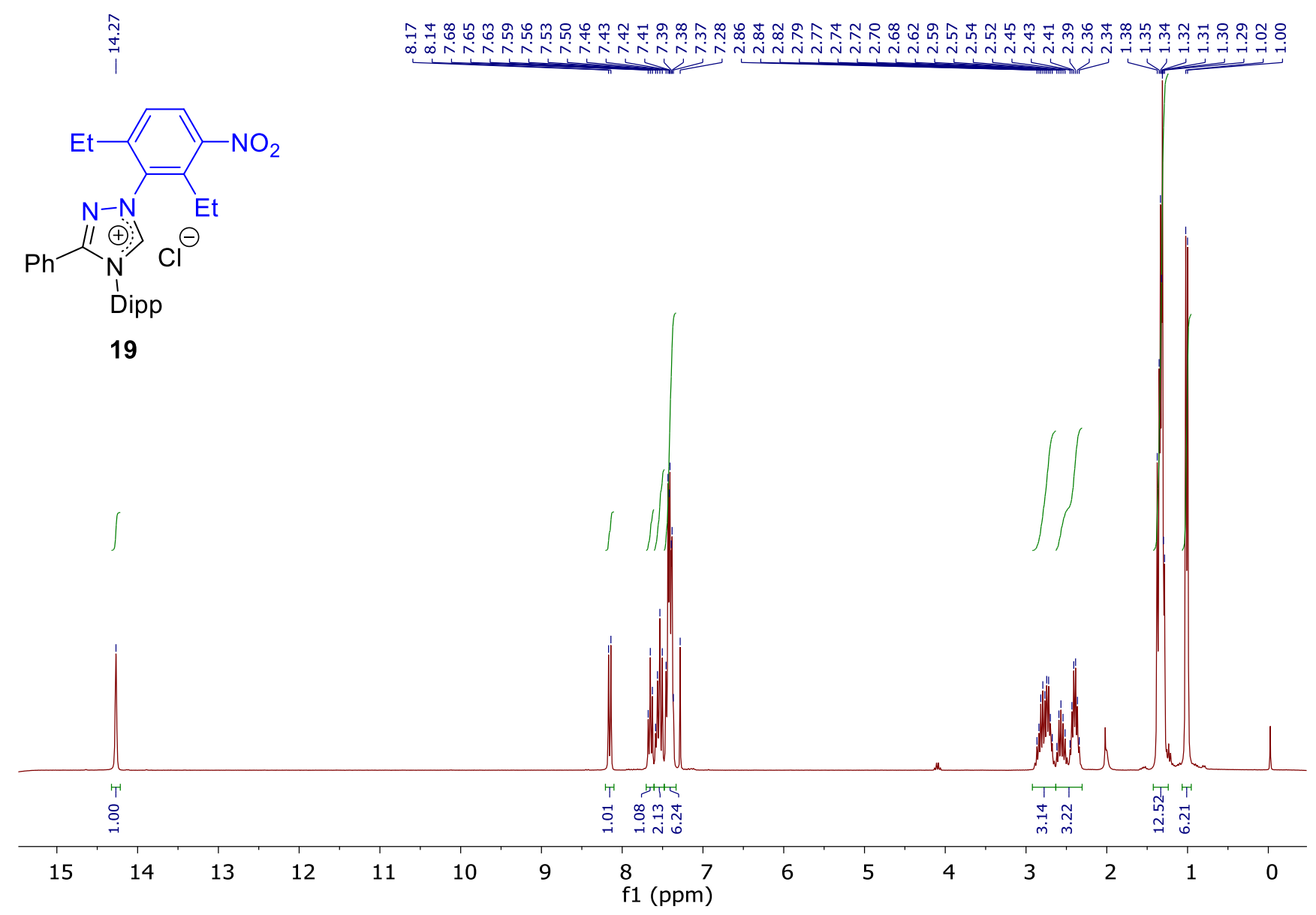

Figure S39. ${ }^{1} \mathrm{H} \mathrm{NMR}\left(300 \mathrm{MHz}, \mathrm{CDCl}_{3}\right)$ of $\mathbf{1 9}$
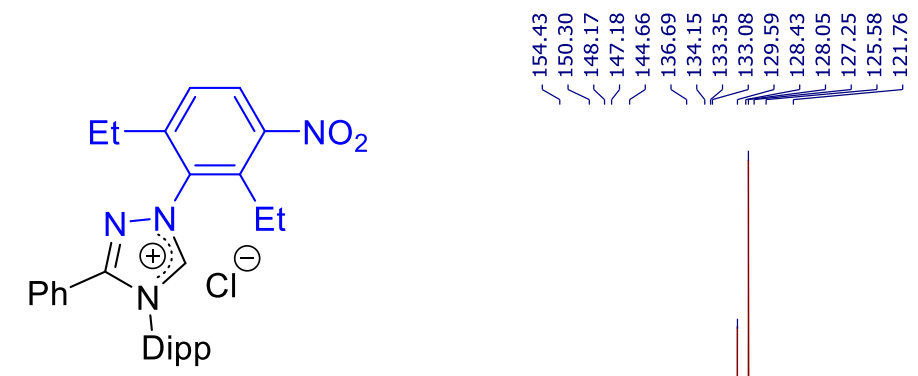

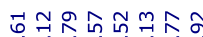

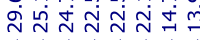

19

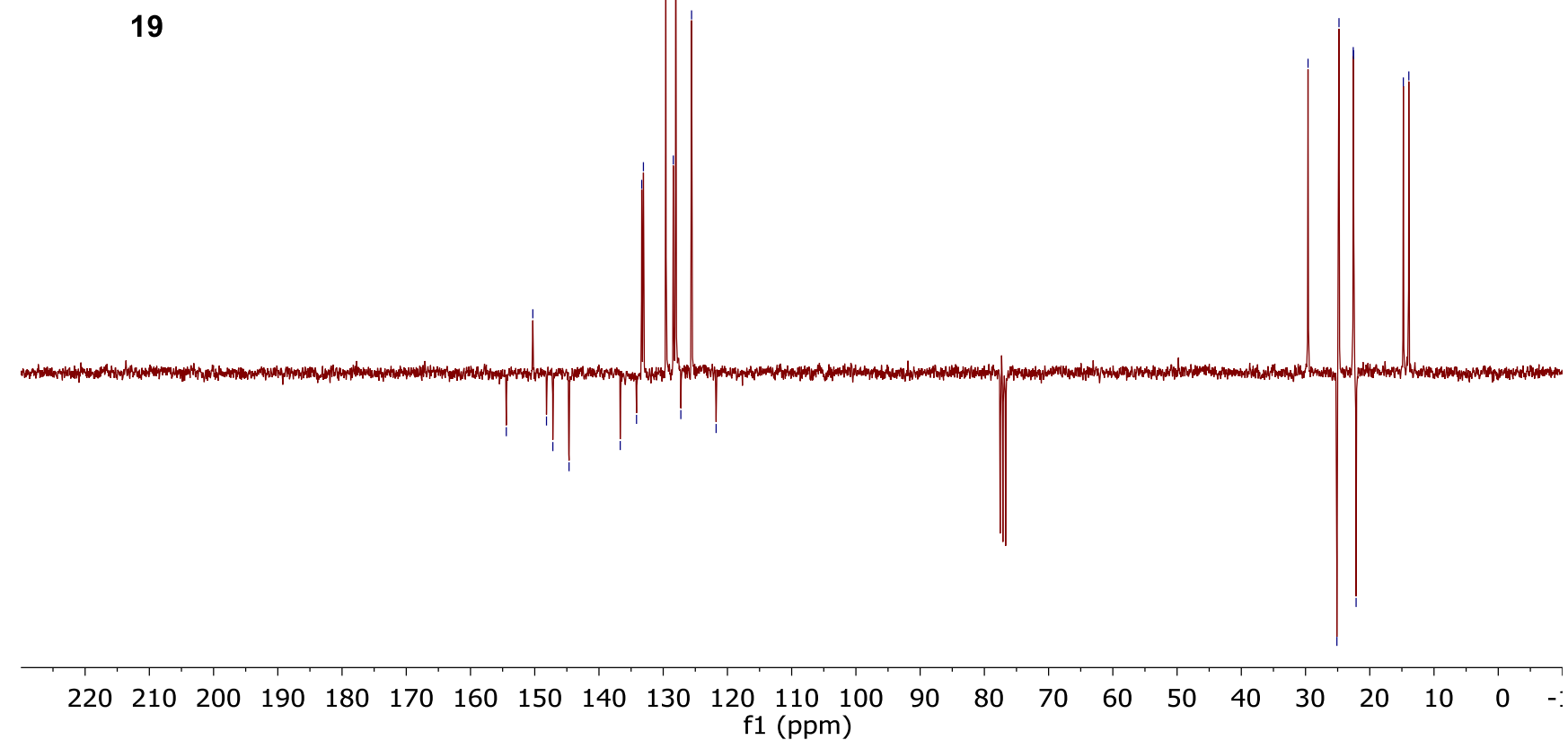

Figure S40. ${ }^{13} \mathrm{C}$ NMR $\left(75 \mathrm{MHz}, \mathrm{APT}, \mathrm{CDCl}_{3}\right)$ of $\mathbf{1 9}$ 
<smiles>CC(C=O)CCCC(C)CC=O</smiles>

(S)-5

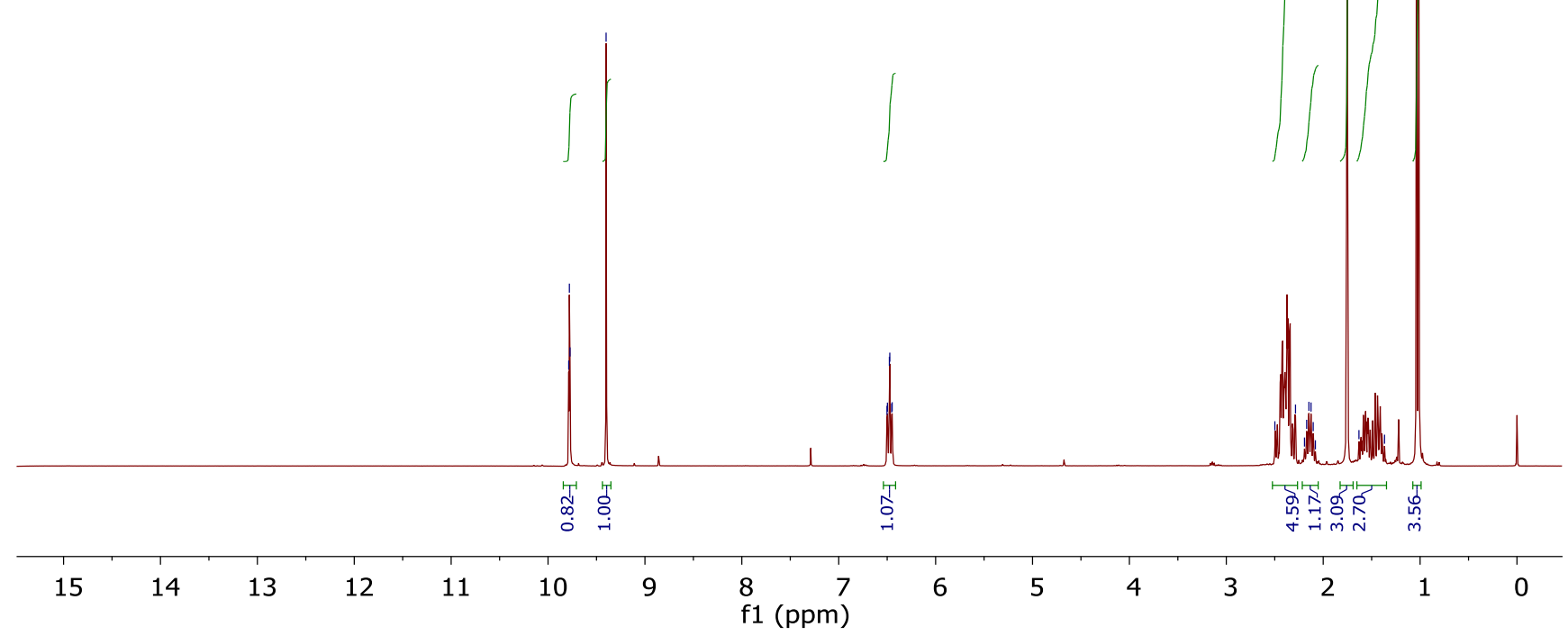

Figure S41. ${ }^{1} \mathrm{H}$ NMR $\left(300 \mathrm{MHz}, \mathrm{CDCl}_{3}\right)$ of $(S)-5$

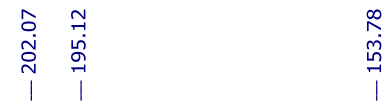<smiles>CC(C=O)CCCC(C)C=CC=O</smiles>

(S) -5

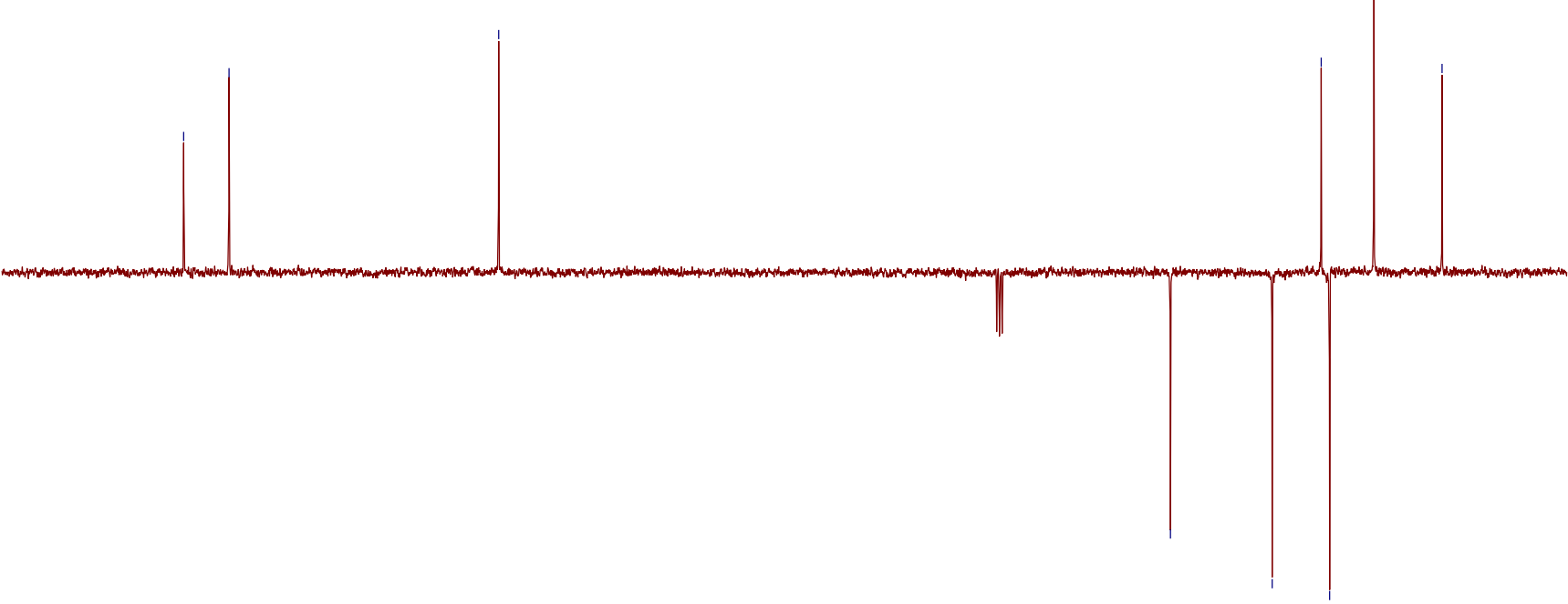
$\begin{array}{lllllllllllllllllllllll}220 & 210 & 200 & 190 & 180 & 170 & 160 & 150 & 140 & 130 & \begin{array}{c}120 \\ \mathrm{f} 1\end{array} \begin{array}{l}110 \\ (\mathrm{ppm})\end{array} & 100 & 90 & 80 & 70 & 60 & 50 & 40 & 30 & 20 & 10 & 0 & -:\end{array}$ Figure $\mathbf{S 4 2} .{ }^{13} \mathrm{C} \mathrm{NMR}\left(75 \mathrm{MHz}, \mathrm{APT}, \mathrm{CDCl}_{3}\right)$ of $(S)-5$ 


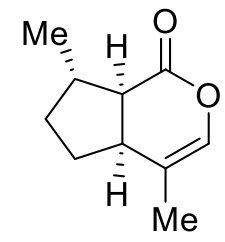

$(+)-1 a$
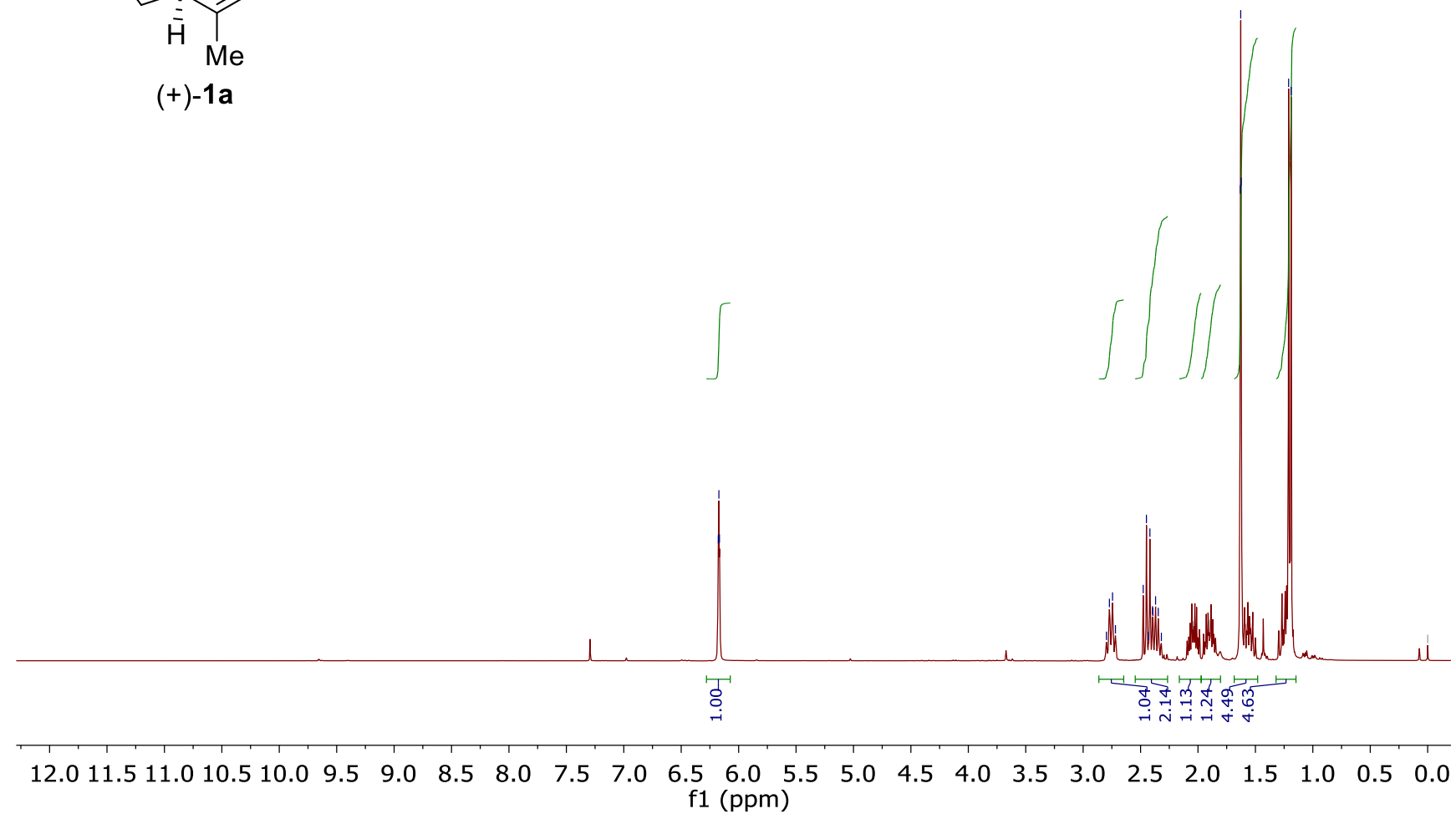

Figure S43. ${ }^{1} \mathrm{H}$ NMR $\left(300 \mathrm{MHz}, \mathrm{CDCl}_{3}\right)$ of (+)-1a<smiles>CC1=COC(=O)[C@H]2C(C)CC[C@@H]12</smiles>

$(+)-1 a$
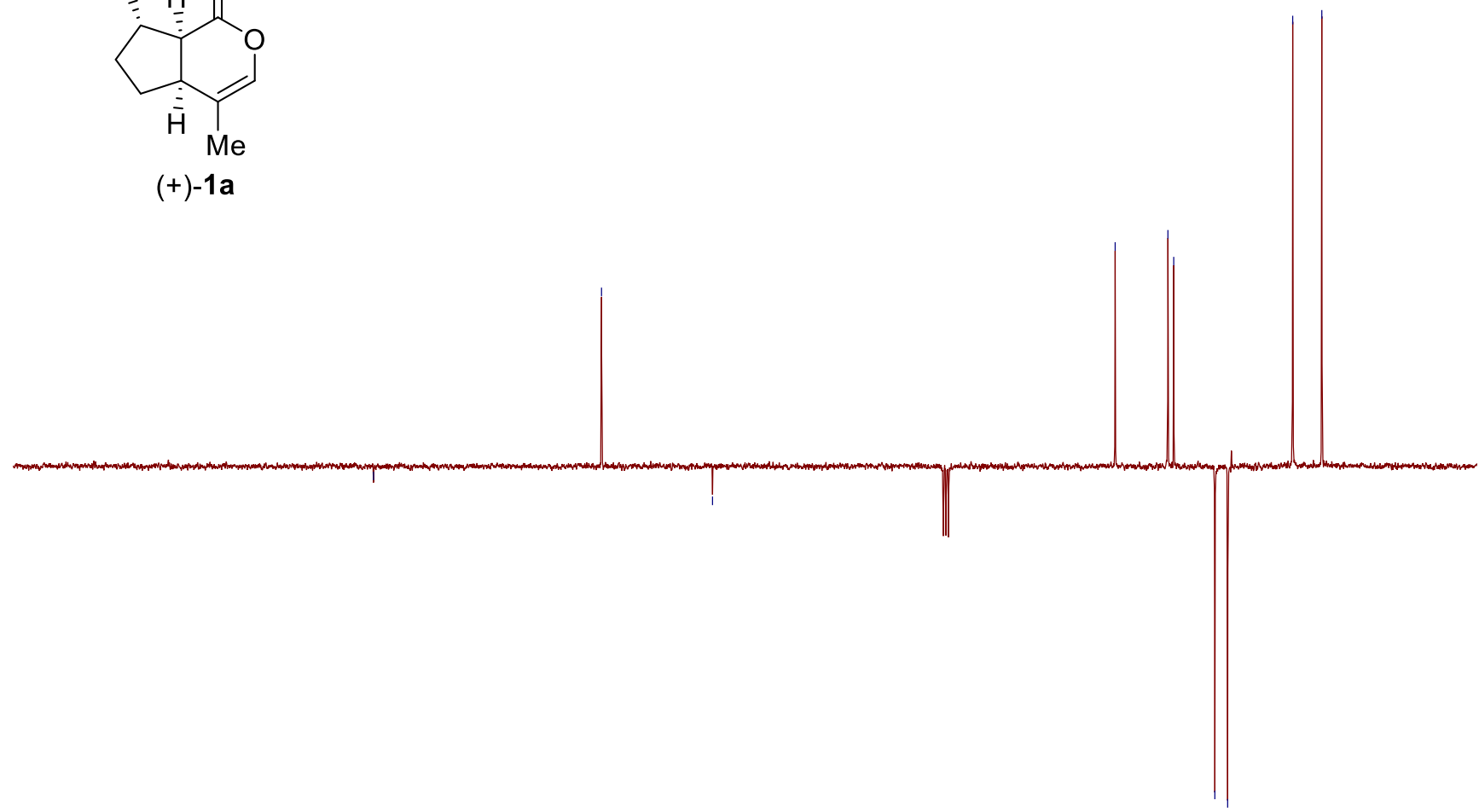

$\begin{array}{llllllllllllllllllllllll}220 & 210 & 200 & 190 & 180 & 170 & 160 & 150 & 140 & 130 & 120 & 110 & 100 & 90 & 80 & 70 & 60 & 50 & 40 & 30 & 20 & 10 & 0 & -\end{array}$ f1 (ppm)

Figure S44. ${ }^{13} \mathrm{C}$ NMR $\left(75 \mathrm{MHz}, \mathrm{APT}, \mathrm{CDCl}_{3}\right)$ of (+)-1a 


\section{X-ray data}

9.1 X-ray data of $\mathbf{1 4}$

Table S1. Crystal data and structure refinement for $\mathbf{1 4}$ (CCDC 1961414)

Identification code

Empirical formula

Moiety formula

Formula weight

Temperature

Wavelength

Crystal system

Space group

Unit cell dimensions

Volume

Z

Density (calculated)

Absorption coefficient

$\mathrm{F}(000)$

Crystal size

Theta range for data collection

Index ranges

Reflections collected

Independent reflections

Completeness to theta $=67.679^{\circ}$

Absorption correction

Max. and min. transmission

Refinement method

Data / restraints / parameters

Goodness-of-fit on $\mathrm{F}^{2}$

Final R indices [I $>2 \operatorname{sigma}(\mathrm{I})]$

$\mathrm{R}$ indices (all data)

Absolute structure parameter

Extinction coefficient

Largest diff. peak and hole nok423_1

$\mathrm{C} 26 \mathrm{H} 27 \mathrm{Cl}$ N4 O2

C26 H27 N4 O2; $\mathrm{Cl}$

462.96

100(2) K

$1.54178 \AA$

Orthorhombic

Pna21

$\begin{array}{ll}\mathrm{a}=22.5200(9) \AA & \alpha=90^{\circ} . \\ \mathrm{b}=7.8346(3) \AA & \beta=90^{\circ} . \\ \mathrm{c}=13.7697(5) \AA & \gamma=90^{\circ} .\end{array}$

2429.46(16) $\AA^{3}$

4

$1.266 \mathrm{Mg} / \mathrm{m}^{3}$

$1.629 \mathrm{~mm}^{-1}$

976

$0.250 \times 0.250 \times 0.050 \mathrm{~mm}^{3}$

3.926 to $72.115^{\circ}$.

$-27<=\mathrm{h}<=27,-9<=\mathrm{k}<=8,-17<=\mathrm{l}<=16$

48295

$4673[\mathrm{R}($ int $)=0.0508]$

$100.0 \%$

Semi-empirical from equivalents

0.7536 and 0.4957

Full-matrix least-squares on $\mathrm{F}^{2}$

4673 / 1 / 308

1.042

$\mathrm{R} 1=0.0245, \mathrm{wR} 2=0.0634$

$\mathrm{R} 1=0.0260, \mathrm{wR} 2=0.0638$

0.052(4)

$\mathrm{n} / \mathrm{a}$

0.184 and -0.217 e. $\AA^{-3}$ 


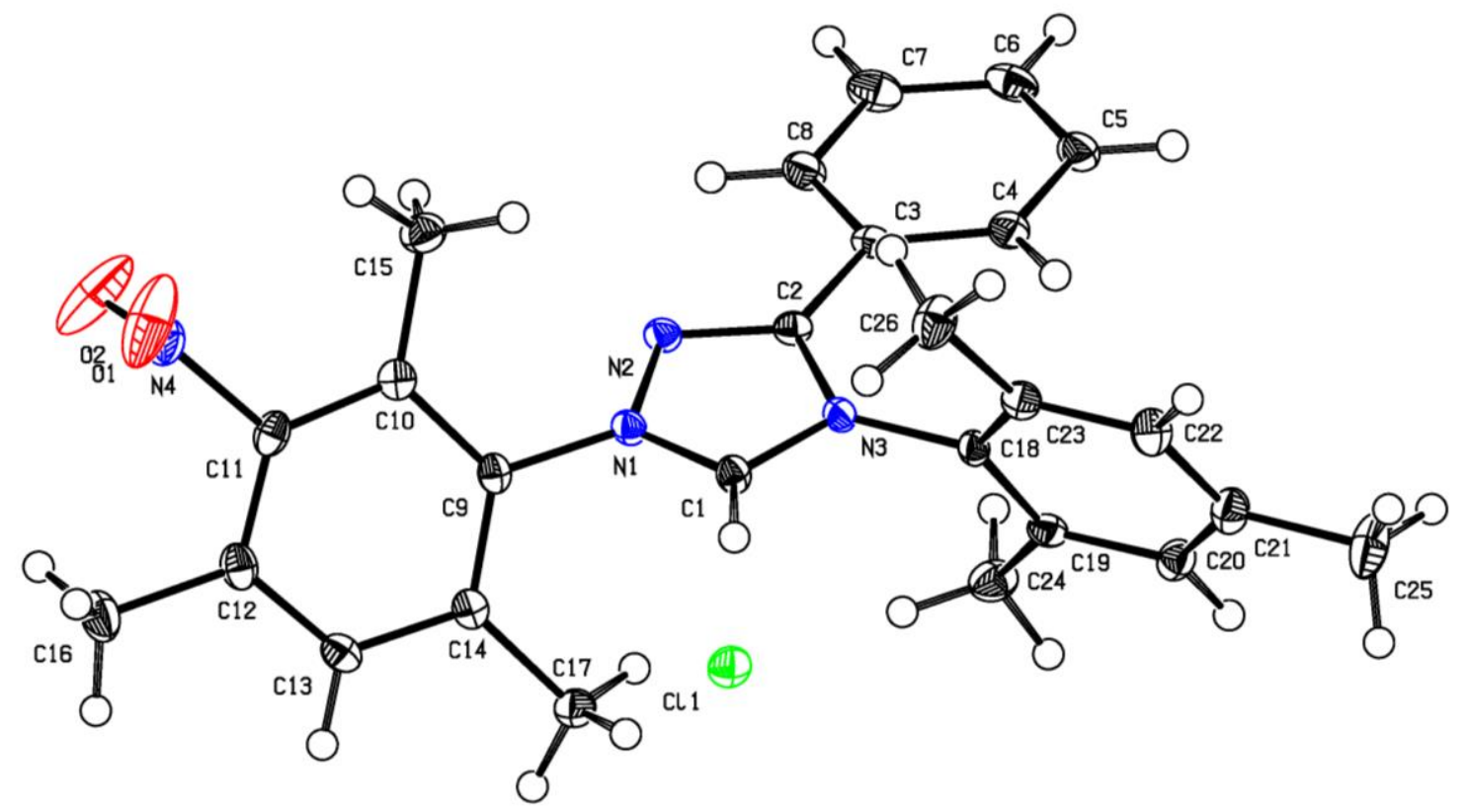

Figure S45. ORTEP of the X-ray crystal structure of 14. Thermal ellipsoids are drawn at $50 \%$ probability level.

\subsection{X-ray data of $\mathbf{1 6}$}

Table S2. Crystal data and structure refinement for 16 (CCDC 1961413)

Identification code

Empirical formula

Moiety formula

Formula weight

Temperature

Wavelength

Crystal system

Space group

Unit cell dimensions

Volume

$\mathrm{Z}$

Density (calculated)

Absorption coefficient

$\mathrm{F}(000)$

Crystal size

Theta range for data collection

Index ranges

Reflections collected

Independent reflections

Completeness to theta $=67.679^{\circ}$ nok_ask18n

$\mathrm{C} 23 \mathrm{H} 21 \mathrm{Cl}$ N4 O2

$\mathrm{C} 23 \mathrm{H} 21 \mathrm{~N} 4 \mathrm{O} 2 ; \mathrm{Cl}$

420.89

100(2) K

$1.54178 \AA$

Triclinic

P-1

$\mathrm{a}=9.0476(3) \AA \quad \alpha=99.0720(10)^{\circ}$.

$\mathrm{b}=11.0348(4) \AA \quad \beta=111.3790(10)^{\circ}$.

$\mathrm{c}=12.0848(4) \AA \quad \gamma=103.8250(10)^{\circ}$.

1050.22(6) $\AA^{3}$

2

$1.331 \mathrm{Mg} / \mathrm{m}^{3}$

$1.832 \mathrm{~mm}^{-1}$

440

$0.200 \times 0.100 \times 0.100 \mathrm{~mm}^{3}$

4.081 to $72.263^{\circ}$.

$-11<=\mathrm{h}<=11,-13<=\mathrm{k}<=13,-14<=\mathrm{l}<=14$

23402

$4116[\mathrm{R}(\mathrm{int})=0.0372]$

$99.7 \%$ 
Absorption correction

Max. and min. transmission

Refinement method

Data / restraints / parameters

Goodness-of-fit on $\mathrm{F}^{2}$

Final R indices [I $>2 \operatorname{sigma}(\mathrm{I})]$

$\mathrm{R}$ indices (all data)

Extinction coefficient

Largest diff. peak and hole
Semi-empirical from equivalents

0.7536 and 0.5916

Full-matrix least-squares on $\mathrm{F}^{2}$

4116 / 0 / 278

1.068

$\mathrm{R} 1=0.0337, \mathrm{wR} 2=0.0895$

$\mathrm{R} 1=0.0366, \mathrm{wR} 2=0.0921$

$\mathrm{n} / \mathrm{a}$

0.224 and -0.323 e. $\AA^{-3}$

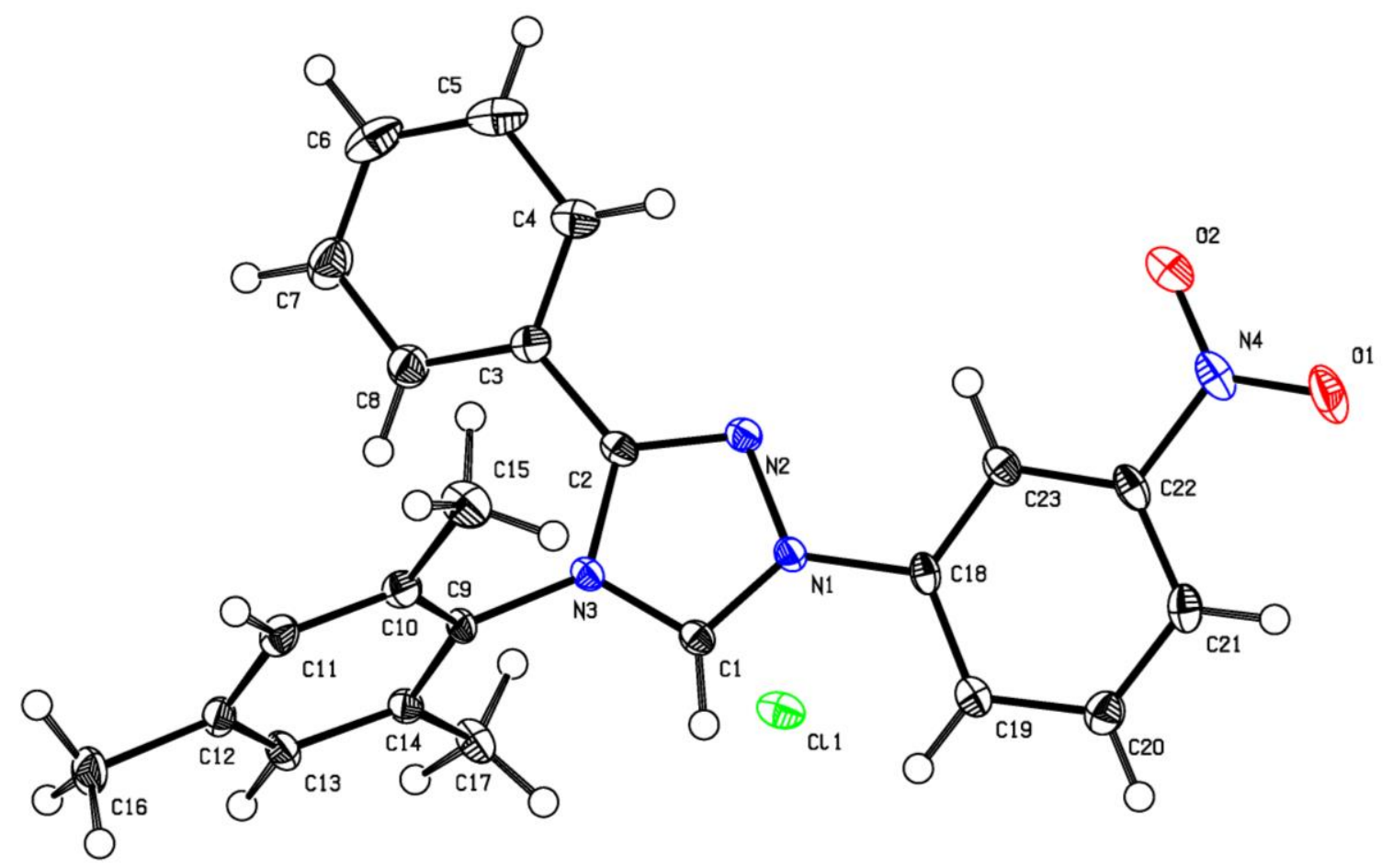

Figure S46. ORTEP of the X-ray crystal structure of 16. Thermal ellipsoids are drawn at $50 \%$ probability level.

\subsection{X-ray data of $\mathbf{1 7}$}

Table S3. Crystal data and structure refinement for 17 (CCDC 1961415)

Identification code

Empirical formula

Moiety formula

Formula weight

Temperature

Wavelength

Crystal system

Space group

Unit cell dimensions
nok427ggn1_CHCl3

$\mathrm{C} 30 \mathrm{H} 34 \mathrm{Cl} 4 \mathrm{~N} 4 \mathrm{O} 2$

C29 H33 N4 O2; Cl; C H Cl3

624.41

100(2) K

$1.54178 \AA$

Triclinic

P-1

$\mathrm{a}=11.3217(7) \AA \quad \alpha=112.3446(18)^{\circ}$.

$\mathrm{b}=11.5130(7) \AA \quad \beta=97.9298(18)^{\circ}$. 
Volume

Z

Density (calculated)

Absorption coefficient

$\mathrm{F}(000)$

Crystal size

Theta range for data collection

Index ranges

Reflections collected

Independent reflections

Completeness to theta $=67.679^{\circ}$

Absorption correction

Max. and min. transmission

Refinement method

Data / restraints / parameters

Goodness-of-fit on $\mathrm{F}^{2}$

Final $\mathrm{R}$ indices [I>2sigma(I)]

$\mathrm{R}$ indices (all data)

Extinction coefficient

Largest diff. peak and hole $\mathrm{c}=13.6237(8) \AA$

$\gamma=103.7307(19)^{\circ}$.
2

$1.344 \mathrm{Mg} / \mathrm{m}^{3}$

$3.756 \mathrm{~mm}^{-1}$

652

$0.400 \times 0.400 \times 0.200 \mathrm{~mm}^{3}$

3.627 to $72.173^{\circ}$.

$-13<=\mathrm{h}<=13,-13<=\mathrm{k}<=14,-16<=\mathrm{l}<=16$

28124

$6005[\mathrm{R}(\mathrm{int})=0.0671]$

$99.7 \%$

Semi-empirical from equivalents

0.7536 and 0.4808

Full-matrix least-squares on $\mathrm{F}^{2}$

6005 / 0 / 369

1.054

$\mathrm{R} 1=0.0401, \mathrm{wR} 2=0.1040$

$\mathrm{R} 1=0.0539, \mathrm{wR} 2=0.1080$

0.0069(4)

0.424 and -0.492 e. $\AA^{-3}$

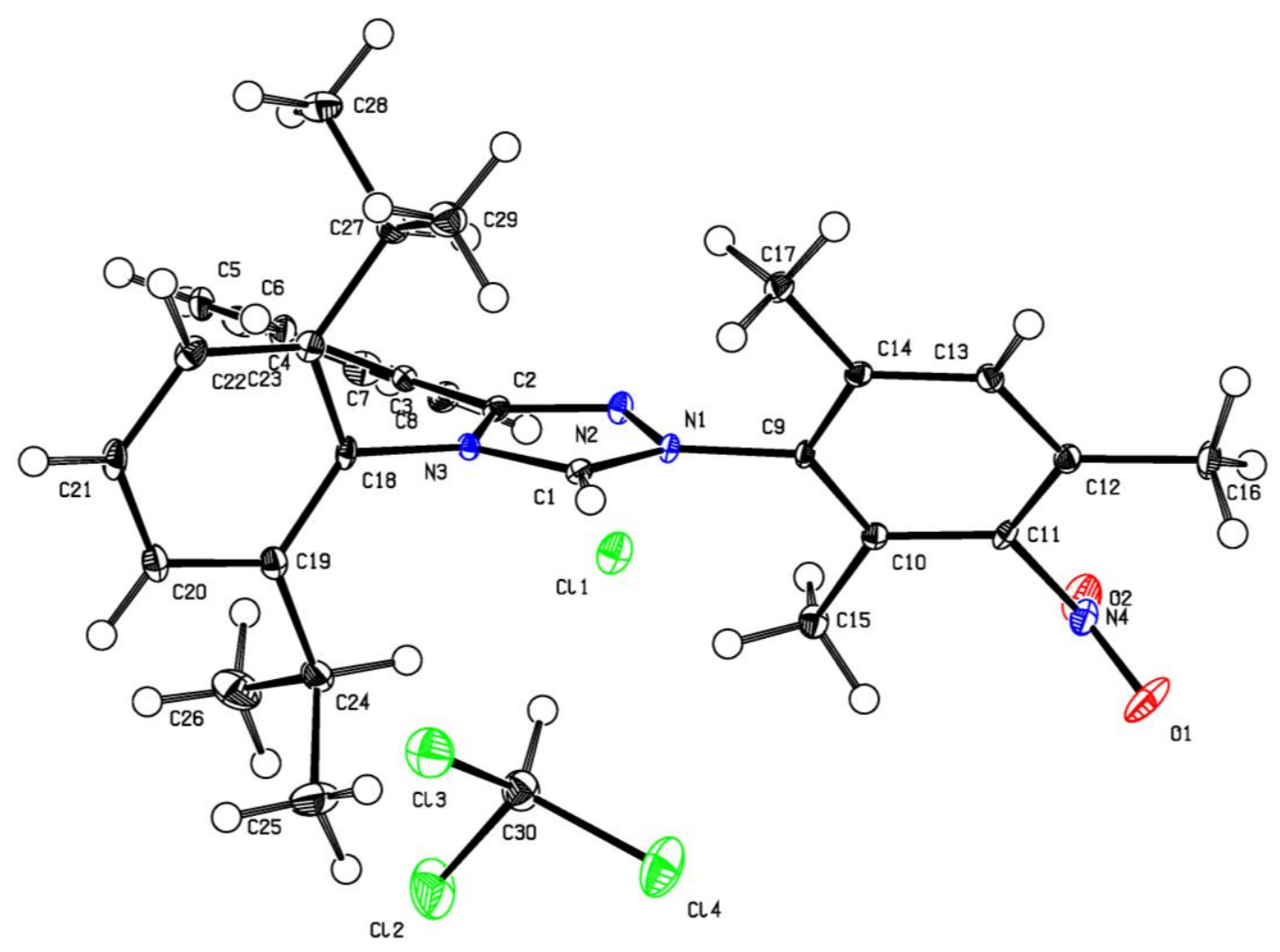

Figure S47. ORTEP of the X-ray crystal structure of $\mathbf{1 7} \bullet \mathrm{CHCl}_{3}$. Thermal ellipsoids are drawn at $50 \%$ probability level. 
9.4 X-ray data of $\mathbf{1 8}$

Table S4. Crystal data and structure refinement for $\mathbf{1 8}$ (CCDC 1961416)

Identification code

Empirical formula

Moiety formula

Formula weight

Temperature

Wavelength

Crystal system

Space group

Unit cell dimensions

Volume

Z

Density (calculated)

Absorption coefficient

$\mathrm{F}(000)$

Crystal size

Theta range for data collection

Index ranges

Reflections collected

Independent reflections

Completeness to theta $=67.679^{\circ}$

Absorption correction

Max. and min. transmission

Refinement method

Data / restraints / parameters

Goodness-of-fit on $\mathrm{F}^{2}$

Final R indices [I $>2 \operatorname{sigma}(\mathrm{I})]$

$\mathrm{R}$ indices (all data)

Extinction coefficient

Largest diff. peak and hole nok380nn

C30 H33 Cl7 N4 O2

C28 H31 Cl N4 O2; "(C H Cl3)

729.75

100(2) K

$1.54178 \AA$

Triclinic

P-1

$$
\begin{array}{ll}
\mathrm{a}=9.6459(8) \AA & \alpha=83.456(3)^{\circ} . \\
\mathrm{b}=12.5634(8) \AA & \beta=76.905(3)^{\circ} . \\
\mathrm{c}=14.9967(14) \AA & \gamma=83.434(2)^{\circ} .
\end{array}
$$$$
\text { 1751.2(2) } \AA^{3}
$$

2

$1.384 \mathrm{Mg} / \mathrm{m}^{3}$

$5.447 \mathrm{~mm}^{-1}$

752

$0.300 \times 0.200 \times 0.050 \mathrm{~mm}^{3}$

3.038 to $72.338^{\circ}$.

$-10<=\mathrm{h}<=11,-15<=\mathrm{k}<=15,-18<=\mathrm{l}<=18$

78799

$6917[\mathrm{R}($ int $)=0.0506]$

$99.9 \%$

Semi-empirical from equivalents

0.7536 and 0.4551

Full-matrix least-squares on $\mathrm{F}^{2}$

6917 / 0 / 398

1.053

$\mathrm{R} 1=0.0417, \mathrm{wR} 2=0.1022$

$\mathrm{R} 1=0.0451, \mathrm{wR} 2=0.1048$

$\mathrm{n} / \mathrm{a}$

0.729 and -0.880 e. $\AA^{-3}$ 


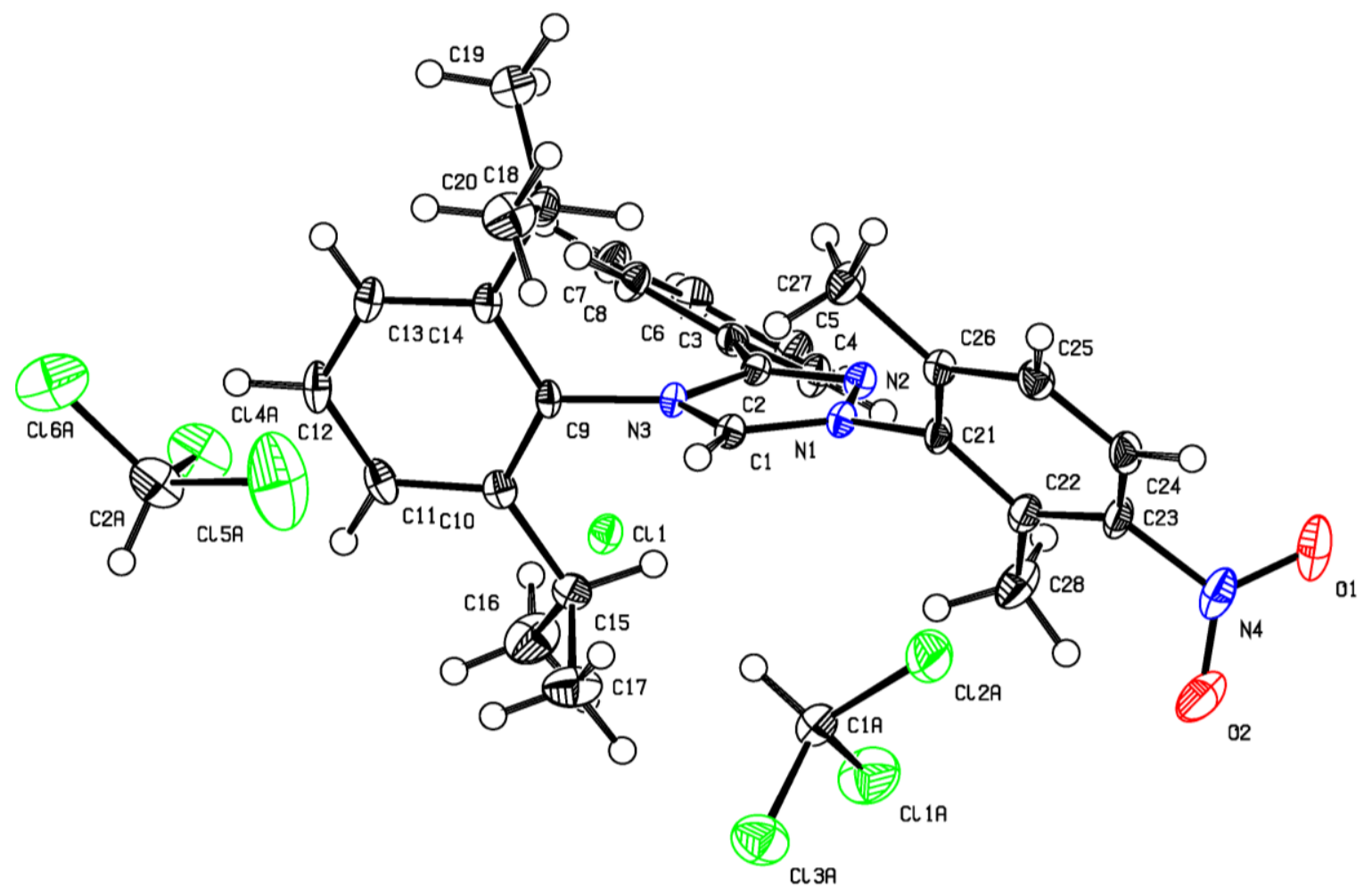

Figure S48. ORTEP of the X-ray crystal structure of $\mathbf{1 8} \bullet 2 \mathrm{CHCl}_{3}$. Thermal ellipsoids are drawn at 50 $\%$ probability level. 